\title{
COMPLEX HYPERBOLIC STRUCTURES ON DISC BUNDLES OVER SURFACES
}

\author{
Sasha Anan'in, Carlos H. Grossi, Nikolay Gusevski
}

\begin{abstract}
We study complex hyperbolic disc bundles over closed orientable surfaces that arise from discrete and faithful representations $H_{n} \rightarrow \mathrm{PU}(2,1)$, where $H_{n}$ is the fundamental group of the orbifold $\mathbb{S}^{2}(2, \ldots, 2)$ and thus contains a surface group as a subgroup of index 2 or 4 . The results obtained provide the first complex hyperbolic disc bundles $M \rightarrow \Sigma$ that

- admit both real and complex hyperbolic structures,

- satisfy the equality $2(\chi+e)=3 \tau$,

- satisfy the inequality $\frac{1}{2} \chi<e$, and

- induce discrete and faithful representations $\pi_{1} \Sigma \rightarrow \mathrm{PU}(2,1)$ with fractional Toledo invariant, where $\chi$ is the Euler characteristic of $\Sigma$, e denotes the Euler number of $M$, and $\tau$ stands for the Toledo invariant of $M$. To get a satisfactory explanation of the equality $2(\chi+e)=3 \tau$, we conjecture that there exists a holomorphic section in all our examples.

In order to reduce the amount of calculations, we systematically explore coordinate-free methods.
\end{abstract}

\section{CONTENTS}

1. Introduction

Outline of the general construction

Acknowledgements

Some notation

2. General construction

2.1. Preliminaries. Cycle of bisectors and the Toledo invariant

2.2. Discreteness

2.3. Simplicity and transversality

2.4. Fibred polyhedra. Euler number

2.5. Transversal triangles

3. Series of explicit examples

3.1. A couple of transversal triangles

3.2. Examples of disc bundles

3.3. Some interesting examples

4. Appendix: technical tools, elementary algebraic and geometric background

4.1. Preliminaries: hermitian metric, complex geodesics, geodesics,

$\mathbb{R}$-planes, and bisectors

4.2. Bisectors

4.3. Bisectors with common slice

4.4. Triangles of bisectors

4.5. Kähler primitive

5. References

\section{Introduction}

This paper is devoted to a particular case of the traditional question concerning the interrelation between topology and geometry: Does a topological 4-manifold admit a specific geometric structure (real hyperbolic, complex hyperbolic, quaternionic, etc.)? Henceforth, $M$ denotes an oriented disc bundle over a closed orientable surface $\Sigma$ of Euler characteristic $\chi$ and $e$ stands for the Euler number of the bundle.

For the existence of a real hyperbolic structure on $M$, there are various conditions in terms of $\chi$ and $e$ (see [GLT], [Kui], [Luo], and also [Kap1, Kap2]). We study the case of complex hyperbolic geometry. In this case, there is one more discrete invariant, the Toledo invariant of the representation $\pi_{1} \Sigma \rightarrow \mathrm{PU}(2,1)$ provided by $M$. It is related to the complex (= Riemannian) structure on $M$, takes

2000 Mathematics Subject Classification. 57S30 (30F35, 51M10, 57M50). 
values in $\frac{2}{3} \mathbb{Z}$, and satisfies the inequality $|\tau| \leq|\chi|$ [Tol]. The Toledo invariant is the only discrete invariant of a (not necessarily discrete) representation $\pi_{1} \Sigma \rightarrow \mathrm{PU}(2,1)$ [Xia].

There are not so many known complex hyperbolic disc bundles. Simple types of such bundles are the $\mathbb{R}$-Fuchsian bundles (they satisfy $e=\chi$ and $\tau=0$ ) and the $\mathbb{C}$-Fuchsian bundles (they are characterized by $\chi=\tau[\mathrm{Tol}]$ and satisfy $\left.e=\frac{1}{2} \chi\right)$. The first complex hyperbolic bundles different from $\mathbb{R}$ - and $\mathbb{C}$ Fuchsian ones were constructed in [GKL]. They satisfy the relations $e=\chi+|\tau / 2|$ and $\chi \leq e \leq \frac{1}{2} \chi$. Thus, $\mathbb{R}$ - and $\mathbb{C}$-Fuchsian bundles provide the extreme values of $e$ in all examples known before the present paper.

Here, we consider a class of discrete and faithful representations that is slightly simpler than that of general representations $\pi_{1} \Sigma \rightarrow \mathrm{PU}(2,1)$. Let $H_{n}$ denote the group generated by $r_{1}, \ldots, r_{n}$ with the defining relations $r_{n} \ldots r_{1}=1$ and $r_{i}^{2}=1, i=1, \ldots, n$. For a hyperelliptic $\Sigma$, the group $H_{n}$ is nothing but the index 2 extension of $\pi_{1} \Sigma$ by the hyperelliptic involution of $\Sigma$ and $n$ is even (for odd $n$, a surface group is a subgroup of index 4 in $\left.H_{n}\right)$. We study discrete and faithful representations $\varrho: H_{n} \rightarrow \mathrm{PU}(2,1)$ such that the $\varrho r_{i}$ 's are reflections in ultraparallel complex geodesics. In order to prove discreteness, we construct fundamental polyhedra bounded by cycles of segments of bisectors (see Outline at the end of Introduction for more details). The main difficulties at these stages are to check that segments of bisectors intersect properly and the fact that adjacent segments have nonconstant angle along their intersection.

The oriented transversal triangles of bisectors studied in Subsections 2.5 and 4.4 serve as building blocks for fundamental polyhedra. The vertices of such a triangle are complex geodesics (= Poincaré discs) naturally equipped with an isometry called the holonomy of the triangle. Every oriented transversal triangle of bisectors bounds a (closed) fibred polyhedron, i.e., a disc bundle over a disc that extends the slice bundle structure of the triangle itself. This fact is established by showing that the space of oriented transversal triangles of bisectors is path-connected and that a triangle of bisectors with common complex spine bounds a fibred polyhedron. It is crucial that the holonomy of a triangle cannot be R-parabolic (see 2.5.1 for the definition) or equal to the identity. Moreover, every oriented transversal triangle of bisectors possesses a 'fractional Euler quantity' that can actually be interpreted as its Euler number. The fractional Euler quantity is an arc on the ideal boundary of a vertex of the triangle. It is determined by the holonomy of the triangle and is additive with respect to gluing triangles.

Gluing a couple of transversal triangles of bisectors, we obtain in Section 3 a quadrangle of bisectors bounding a fundamental polyhedron for the group generated by $U, W \in \mathrm{PU}(2,1)$ with the relations $U^{n}=W^{n}=\left(U^{-1} W\right)^{2}=1$. The quadrangle furnishes a complex hyperbolic disc bundle over the turnover orbifold $\mathbb{S}^{2}(n, n, 2)$ whose rational Euler number (see [BSi] for the definition) can be inferred from the fractional Euler quantities of the involved triangles. Since $H_{n}$ is a subgroup of index $n$ in the turnover group, we get a complex hyperbolic disc bundle $M$ over a closed surface. Clearly, we simultaneously obtain a compact 3-manifold (a circle bundle over a closed surface) admitting a spherical CR structure.

In the above way, we construct a huge family of explicit examples of disc bundles $M$. All of them satisfy the inequality $\frac{1}{2} \chi<e$ and the equality $2(\chi+e)=3 \tau$ (with negative $\chi, e, \tau$ ). The inequality was never valid for previously known examples and the equality was valid only in the $\mathbb{C}$-Fuchsian case. Since the equality is a necessary condition for the existence of a holomorphic section of $M$ (in the sense that there are a disc bundle structure on $M$ and a smooth holomorphic surface $\Sigma \subset M$ that intersects every fibre exactly once), we conjecture that there exists a holomorphic section in all our examples. For many examples, $\tau$ is not integer, which implies in particular that the corresponding representation $\pi_{1} \Sigma \rightarrow \mathrm{PU}(2,1)$ cannot be lifted to $\mathrm{SU}(2,1)$. We obtain the first disc bundles $M$ admitting both real and complex hyperbolic structures. Passing to the corresponding circle bundles, we see that there exist circle bundles over closed orientable surfaces admitting simultaneously conformally flat and spherical CR structures. For a more detailed discussion about the features of our examples and related conjectures, see Subsection 3.3. 
Whenever reasonable, we work without coordinates. This concerns especially the appendix (Section 4) which is devoted to an exposition of algebraic and geometric background and contains the proofs absent in Sections 2-3. We believe that some facts in the appendix are interesting per se. This includes: explicit formulae for the normal vector to an oriented bisector (Proposition 4.2.11) and for the angle between two cotranchal bisectors (Lemma 4.3.1); a numerical transversality criterion for a pair of cotranchal bisectors (Criterion 4.3.3); a sort of metric separability of transversal cotranchal bisectors (Lemma 4.3.6) that is a key point allowing us to apply Poincaré's Polyhedron Theorem; and an explicit formula for a Kähler primitive in 4.5.1.

The reader unfamiliar with complex hyperbolic geometry may begin reading with Section 4 . The reader familiar with complex hyperbolic geometry may first read Sections 2-3 (the geometric core of the paper).

Outline of the general construction. Roughly speaking, the general construction of a complex hyperbolic disc bundle $M$ deals with a certain fundamental polyhedron $F$ for the group $\pi_{1} M \subset \mathrm{PU}(2,1)$. The polyhedron $F$ is bounded by a cycle of oriented segments of bisectors $\left(\mathrm{B}_{1}, \ldots, \mathrm{B}_{m}\right)$ such that the final slice of $\mathrm{B}_{i}$ is the initial slice of $\mathrm{B}_{i+1}$ for every $i$ (the indices are modulo $m$ ). Suppose that the obvious slice bundle structure of the boundary $\partial_{0} F:=\mathrm{B}_{1} \cup \cdots \cup \mathrm{B}_{m}$ of $F$ is extendable to $F$. Then, taking into account that the identifications of the segments by means of elements in $\pi_{1} M$ preserve the slice bundle structure of $\partial_{0} F$, we obtain a complex hyperbolic disc bundle $M$.

For technical reasons, the polyhedron $F$ is glued from two or four polyhedra $P$, each bounded by a cycle $\mathcal{C}$ of segments $\left(\mathrm{B}_{1}, \ldots, \mathrm{B}_{n}\right)$. The identification of faces of $P$ is given by the reflections $R_{i}$ in the middle slices $M_{i}$ of the $\mathrm{B}_{i}$ 's which satisfy the relation $R_{n} \ldots R_{1}=1$. If we show that $P$ is a fundamental polyhedron for the group $H$ generated by the $R_{i}$ 's and that the slice bundle structure of $\partial_{0} P$ is extendable to $P$, then we arrive at the desired complex hyperbolic disc bundle $M$.

The solid torus $\partial_{0} P$ can be readily fibred into circles called meridional curves of $\mathcal{C}$ : in its segment $b_{i}$ belonging to $\mathrm{B}_{i}$, every meridional curve $b$ is equidistant from the real spine of $\mathrm{B}_{i}$ and lies in a suitable meridian of $\mathrm{B}_{i}$. Moreover, $R_{i} b_{i}=b_{i}$ and $R_{i}$ acts on $b_{i}$ as a 'reflection' in the middle point of $b_{i}$, i.e., in the point $b_{i} \cap M_{i}$. In other words, the reflections $R_{i}$ 's preserve the fibering of $\partial_{0} P$ by meridional curves. Every meridional curve of the cycle $\mathcal{C}$ is intended to generate a section of the disc bundle, i.e., to be the boundary of a disc that provides a section.

Considering those 'formally' adjacent polyhedra congruent to $P$ that are intended to tessellate a neighbourhood of the slice $S_{i+1}$ common to the segments $\mathrm{B}_{i}$ and $\mathrm{B}_{i+1}$, we can see that the sum of the interior angles of these adjacent polyhedra at a point $s \in S_{i+1}$ (called the total angle at $s$ ) is an integer multiple of $2 \pi$ and that the total angle at $s$ is independent of the choice of $s$ in $S_{i+1}$. Requiring that the total angle is $2 \pi$ and that the full bisectors $\prec \mathrm{B}_{i} \succ$ and $\prec \mathrm{B}_{i+1} \succ$ of the segments $\mathrm{B}_{i}$ and $\mathrm{B}_{i+1}$ are transversal along their common slice $S_{i+1}$ for all $i$ (such a cycle $\mathcal{C}$ is said to be simple transversal with total angle $2 \pi$ ), we show that $P$ is indeed a fundamental polyhedron for $H$.

It is not so easy to construct a simple transversal cycle of bisectors. The problem is that the segments $\mathrm{B}_{i}$ and $\mathrm{B}_{j}$ can unexpectedly intersect, that is, the cycle $\mathcal{C}$ can be nonsimple and, thus, can provide no polyhedron $P$. Under some additional requirement (appealing to some kind of simple Poincaré duality), we show that the cycle is simple.

In general, we cannot expect the slice bundle structure of $\partial_{0} P$ to be extendable to $P$. It is quite possible that the polyhedron $P$ is 'knotted.' Although this is a topological problem, it looks even more difficult than the problem of simplicity because it requires to dwell into the mutual position of all the $\mathrm{B}_{i}$ 's (not only to verify that the $\mathrm{B}_{i}$ 's intersect properly). An easy way to avoid such a situation is to show that the polyhedron $P$ can be cut into some simple polyhedra fibred in a way compatible with gluing them back. Besides, this helps to solve the problem of the simplicity of a cycle. Transversal triangles of bisectors are good candidates for such simple fibred polyhedra. 
Acknowledgements. The authors are grateful to Victor Gerasimov for the discussions in the beginning of this work. We are indebted to Misha Kapovich for his interest in our work and many important suggestions that considerably improved the exposition. We express our gratitude to the referee for many helpful comments. We thank Israel Vainsencher for a valuable remark. This article is dedicated to the memory of Igor' Vladimirovich L'vov.

\section{Some notation}

\begin{tabular}{|c|c|c|c|c|}
\hline 2.1 .14 & $\mathrm{~B}\left[S, S^{\prime}\right]$ & $e_{P} \mathbf{2 . 4 . 6} 13$ & 2.3.1 10 & 2.1 .35 \\
\hline 2.1 .14 & $\mathrm{~B}\left[p_{1}, p_{2}\right] \quad \mathbf{2 . 3 . 1} 10$ & G $S \quad 4.1 .931$ & 4.1.9 31 & $\mathrm{R} S \mathbf{4 . 1 . 9} 31$ \\
\hline 2.2.3 9 & $\mathrm{~B}\left\langle g_{1}, g_{2} \nmid \quad \mathbf{4 . 1 . 2 0} 33\right.$ & $\mathrm{G}\left[p_{1}, p_{2}\right] \quad \mathbf{2 . 3 . 1} 10$ & $\mathrm{~L}\left\langle p_{1}, p_{2}\right\} \quad \mathbf{4 . 1 . 6} 30$ & $\operatorname{ta}\left(p_{1}, p_{2}\right) \quad 2.1 .24$ \\
\hline$\succ \mathbf{2 . 3 . 1} 10$ & $\partial_{0} \quad \mathbf{2 . 2 . 2} 9$ & $\mathrm{G}\left\langle p_{1}, p_{2}\right\} \quad \mathbf{4 . 1 . 1 1} 31$ & $\pi^{\prime}[p] v \quad \mathbf{4 . 1 . 1} 29$ & $t_{\varphi} \mathbf{2 . 1 . 1 1 7}$ \\
\hline$B \succ \mathbf{2 . 3 . 1} 10$ & $\partial_{\infty} \mathbf{2 . 1 . 1 4}$ & $K^{+} \mathbf{2 . 3 . 1} 10$ & $\pi[p] v \quad \mathbf{4 . 1 . 1} 29$ & $v_{p} \mathbf{2 . 1 . 1 1} 7$ \\
\hline
\end{tabular}

\section{General construction}

In this section, we develop some general tools applicable to constructing complex hyperbolic disc bundles over closed orientable surfaces.

\subsection{Preliminaries. Cycle of bisectors and the Toledo invariant}

In this subsection, we introduce some basic notation, conventions, and simple facts. Then we define configurations and cycles of bisectors, meridional curves, and related concepts. Finally, we calculate the Toledo invariant of representations defined by cycles of bisectors.

2.1.1. Fix, once and for all, a three-dimensional $\mathbb{C}$-vector space $V$ equipped with a hermitian form $\langle-,-\rangle$ of signature ++- . The corresponding unitary, special unitary, and projective unitary groups are respectively denoted by U, SU, and PU. Depending on the context, we will use the elements of $V$ to denote the points in the complex projectivization $\mathbb{P}$ of $V$. In general, $\mathbb{P} W$ denotes the complex projectivization of $W \subset V$.

It is well known that the complex hyperbolic plane can be identified with the open 4 -ball ${ }^{1}$

$$
\mathbb{B}:=\{p \in \mathbb{P} \mid\langle p, p\rangle<0\} .
$$

The complex hyperbolic distance between two points $p_{1}, p_{2} \in \mathbb{B}$ can be expressed in terms of the tance

$$
\operatorname{ta}\left(p_{1}, p_{2}\right):=\frac{\left\langle p_{1}, p_{2}\right\rangle\left\langle p_{2}, p_{1}\right\rangle}{\left\langle p_{1}, p_{1}\right\rangle\left\langle p_{2}, p_{2}\right\rangle}
$$

as explained in [Gol, p. 77]. Sometimes, it is convenient to use the tance instead of the distance: the latter is a monotonic function of the former, whereas the tance has an algebraic nature and a simpler form. Furthermore, the tance is involved in many complex hyperbolic concepts.

The ideal boundary of the complex hyperbolic plane is the 3 -sphere

$$
\partial_{\infty} \mathbb{B}:=\{p \in \mathbb{P} \mid\langle p, p\rangle=0\}
$$

formed by all isotropic points. We denote $\overline{\mathbb{B}}:=\mathbb{B} \cup \partial_{\infty} \mathbb{B}$. Preferentially, we consider geometrical objects such as geodesics, complex geodesics, $\mathbb{R}$-planes, bisectors, etc. as being closed in $\overline{\mathbb{B}}$. The exceptions occur when explicitly stated or when a concept or formula is clearly inapplicable to isotropic points. In general, $\partial_{\infty} X$ stands for $\partial_{\infty} \mathbb{B} \cap X$.

\footnotetext{
${ }^{1}$ Here and in what follows, the symbol := stands for 'equals by definition.'
} 
For every nonisotropic $p \in \mathbb{P}$, define the linear map

$$
R(p): x \mapsto 2 \frac{\langle x, p\rangle}{\langle p, p\rangle} p-x .
$$

Such a definition provides the reflection $R(p) \in \mathrm{SU}$. If $p$ is positive, that is, if $p \notin \overline{\mathbb{B}}$, then $R(p)$ is the reflection in the complex geodesic $\mathbb{P} p^{\perp} \cap \overline{\mathbb{B}}$, where $p^{\perp}:=\{x \in V \mid\langle x, p\rangle=0\}$. The point $p$ is said to be the polar point to $\mathbb{P} p^{\perp}$. For brevity, $\mathbb{P} p^{\perp}$ will denote both the complex geodesic $\mathbb{P} p^{\perp} \cap \overline{\mathbb{B}}$ and its projective line, depending on the context.

It is well known that every bisector in $\bar{B}$ admits two decompositions into totally geodesic 2-planes: the slice decomposition into complex geodesics and the meridional decomposition into $\mathbb{R}$-planes (see [Gol, p. 152]). We treat every bisector as being oriented by an orientation of its real spine and the natural (complex) orientation of its slices. Let $S$ and $S^{\prime}$ be two complex geodesics. The inequality $\operatorname{ta}\left(p, p^{\prime}\right)>1$ means that $S$ and $S^{\prime}$ are ultraparallel [Gol, p. 100], where $p$ and $p^{\prime}$ stand for the polar points to $S$ and $S^{\prime}$. In this case, there exists a unique bisector $\mathcal{B}$ containing $S$ and $S^{\prime}$ as slices [Gol, p. 165, Theorem 5.2.4]. We denote by $\mathrm{B}\left[S, S^{\prime}\right] \subset \mathcal{B}$ the closed oriented segment of bisector formed by the slices from $S$ to $S^{\prime}$ and call $\mathcal{B}$ the full bisector of the segment $\mathrm{B}\left[S, S^{\prime}\right]$. It is easy to see that there exists a unique slice $M$ of $\mathrm{B}\left[S, S^{\prime}\right]$ such that the reflection in $M$ exchanges $S$ and $S^{\prime}$. We call $M$ the middle slice of $\mathrm{B}\left[S, S^{\prime}\right]$.

Let $\mathrm{B}\left[S, S^{\prime}\right]$ be an oriented segment of bisector and let $s \in S$. We take a meridian $\mathrm{R}$ of $\mathrm{B}\left[S, S^{\prime}\right]$ such that $s \in \mathrm{R}$. Let $b$ denote the curve contained in $\mathrm{R}$ that begins at $s \in S$, ends at some point $s^{\prime} \in S^{\prime}$, and is equidistant from the real spine of the bisector ( $b$ is a segment of a hypercycle in $\mathrm{R}$ ). We call $b$ the meridional curve of $\mathrm{B}\left[S, S^{\prime}\right]$ generated by $s \in S$. When $s \in \partial_{\infty} S$, the ideal meridional curve $b$ is defined as the segment of the $\mathbb{R}$-circle in $\mathrm{B}\left[S, S^{\prime}\right]$ that contains $s$.

Lemma 2.1.4. Let $\mathrm{B}\left[S, S^{\prime}\right]$ be an oriented segment of bisector and let $b$ be an (ideal) meridional curve of $\mathrm{B}\left[S, S^{\prime}\right]$ that begins at $s \in S$ and ends at $s^{\prime} \in S^{\prime}$. Then $R b=b$ and $R s=s^{\prime}$, where $R$ stands for the reflection in the middle slice of $\mathrm{B}\left[S, S^{\prime}\right]$.

Proof. There is a meridian $\mathrm{R}$ of $\mathrm{B}\left[S, S^{\prime}\right]$ such that $b \subset \mathrm{R}$. The reflection in the middle slice $M$ of $\mathrm{B}\left[S, S^{\prime}\right]$, being restricted to $\mathrm{R}$, is the reflection in the geodesic $\mathrm{R} \cap M$ that is orthogonal to the real spine of $\mathrm{B}\left[S, S^{\prime}\right]$.

Lemma 2.1.5 [Appendix, Lemma 4.2.16]. An (ideal) meridional curve depends continuously on the segment of bisector and on the initial point of the curve.

2.1.6. Let $S_{1}, \ldots, S_{n}$ be complex geodesics such that $S_{i}$ and $S_{i+1}$ are ultraparallel for all $i$ (the indices are modulo $n$ ). Denote $\mathrm{B}_{i}:=\mathrm{B}\left[S_{i}, S_{i+1}\right]$. We call $\left(\mathrm{B}_{1}, \ldots, \mathrm{B}_{n}\right)$ a configuration of bisectors. For every $\mathrm{B}_{i}$, we denote by $M_{i}$ the middle slice of $\mathrm{B}_{i}$ and by $R_{i}$, the reflection in $M_{i}$.

A configuration of bisectors $\left(\mathrm{B}_{1}, \ldots, \mathrm{B}_{n}\right)$ is called simple if $\mathrm{B}_{i} \cap \mathrm{B}_{j} \neq \varnothing$ implies that $j \in\{i-1, i, i+1\}$ and if $\mathrm{B}_{i-1} \cap \mathrm{B}_{i}=S_{i}$ for all $i$. A configuration is transversal if, for all $i, \mathrm{~B}_{i-1} \cap \mathrm{B}_{i}=S_{i}$ and the full bisectors of $\mathrm{B}_{i-1}$ and $\mathrm{B}_{i}$ are transversal along $S_{i}$ (this includes transversality along $\partial_{\infty} S_{i}$ ).

Definition 2.1.7. A cycle of bisectors is a configuration of bisectors $\left(\mathrm{B}_{1}, \ldots, \mathrm{B}_{n}\right)$ such that the reflections $R_{i}$ 's in the middle slices $M_{i}$ 's satisfy the relation $R_{n} \ldots R_{1}=1$ in PU. In terms of SU, identifying the centre of $\mathrm{U}$ with the unit complex numbers, we have $R_{n} \ldots R_{1}=\delta$, where $\delta \in \mathbb{C}$ and $\delta^{3}=1$. We denote by $H:=\left\langle R_{i} \mid 1 \leq i \leq n\right\rangle$ the subgroup in PU generated by the $R_{i}$ 's.

Given reflections $R_{1}, \ldots, R_{n}$ in complex geodesics $M_{1}, \ldots, M_{n}$ subject to the relation $R_{n} \ldots R_{1}=1$ in PU, a cycle of bisectors can be constructed as follows. Let $S_{1}$ be a complex geodesic. Define $S_{i+1}:=R_{i} S_{i}$. Requiring that $M_{i}$ and $S_{i}$ are ultraparallel for all $i$, we obtain the segments $\mathrm{B}_{i}:=$ $\mathrm{B}\left[S_{i}, S_{i+1}\right]$ forming the cycle $\left(\mathrm{B}_{1}, \ldots, \mathrm{B}_{n}\right)$. Constructing a cycle in this way does depend on the choice of $S_{1}$. There are many suitable choices for $S_{1}$. Indeed, for some positive points $m_{i}$ and $p_{1}$, we have 
$R_{i}=R\left(m_{i}\right), M_{i}=\mathbb{P} m_{i}^{\perp}, S_{1}=\mathbb{P} p_{1}^{\perp}$, and $S_{i}:=\mathbb{P} p_{i}^{\perp}$, where $p_{i+1}:=R_{i} p_{i}$. In these terms, the inequality $\operatorname{ta}\left(m_{i}, p_{i}\right)>1$ says that $M_{i}$ and $S_{i}$ are ultraparallel. We have $\operatorname{ta}\left(m_{i}, p_{i}\right)=\operatorname{ta}\left(R_{1} \ldots R_{i-2} R_{i-1} m_{i}, p_{1}\right)$. All these tances tend to infinity while $p_{1} \notin \overline{\mathbb{B}}$ tends to a generic point in $\partial_{\infty} \mathbb{B}$.

2.1.8. Meridional curve of a cycle. Let $\left(\mathrm{B}_{1}, \ldots, \mathrm{B}_{n}\right)$ be a configuration of bisectors. Take $s_{1} \in S_{1}$. The point $s_{1}$ generates a meridional curve $b_{1}$ of $\mathrm{B}_{1}$ which ends at some $s_{2} \in S_{2}$. By Lemma 2.1.4, $R_{1} b_{1}=b_{1}$ and $R_{1}$ exchanges $s_{1}$ and $s_{2}$. Inductively, $s_{i}$ generates the meridional curve $b_{i}$ of $\mathrm{B}_{i}$ which ends at some $s_{i+1} \in S_{i+1}$. Again, $R_{i} b_{i}=b_{i}, R_{i} s_{i}=s_{i+1}$, and $R_{i} s_{i+1}=s_{i}$. We call ${ }^{2}$ the curve $b:=b_{1} \cup \cdots \cup b_{n}$ the meridional curve of the configuration generated by $s_{1}$. Similarly, $s_{1} \in \partial_{\infty} S_{1}$ generates an ideal meridional curve. The $\operatorname{arcs} b_{1}, \ldots, b_{n}$ are edges and the points $s_{1}, \ldots, s_{n}$ are vertices of the (ideal) meridional curve. In the case of a cycle, the meridional curves are closed due to the relation $R_{n} \ldots R_{1}=1$, as shown in the picture.

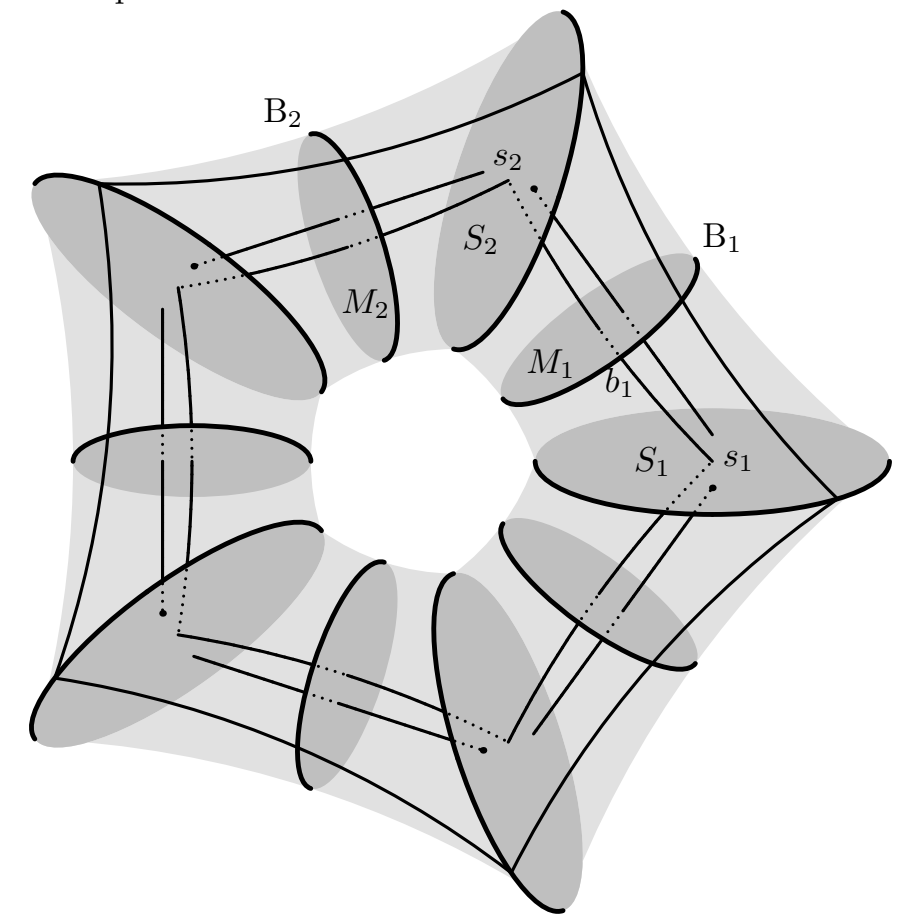

2.1.9. Planar example. The concept of a cycle of bisectors in $\mathbb{B}$ originates from the following planar example.

Fix an integer $n \geq 5$. Let $P$ be a simply connected geodesic $n$-gon in the real hyperbolic plane $\mathbb{H}_{\mathbb{R}}^{2}$ with vertices $v_{1}, \ldots, v_{n}$ and interior angles $\alpha_{1}, \ldots, \alpha_{n}$ such that $\alpha_{1}+\cdots+\alpha_{n}=2 \pi$. We denote the edges of $P$ by $e_{1}, \ldots, e_{n}$, where $e_{i}$ connects $v_{i}$ and $v_{i+1}$ (the indices are modulo $n$ ). Let $r_{i}$ stand for the reflection in the middle point of $e_{i}$. By Poincaré's Polyhedron Theorem, $P$ provides a fundamental domain for the group

$$
\left.H_{n}:=\left\langle r_{1}, \ldots, r_{n}\right| r_{n} \ldots r_{1}=1 \text { and } r_{i}^{2}=1\right\rangle
$$

generated by the $r_{i}$ 's subject to the defining relations $r_{n} \ldots r_{1}=1$ and $r_{i}^{2}=1$. The group $H_{n}$ is the fundamental group of the sphere with $n$ elliptic points of order 2 , i.e., of the orbifold $\mathbb{S}^{2}(2, \ldots, 2)$. Passing to a torsion-free subgroup of finite index, we arrive at surface groups as follows.

For even $n, P \cup r_{1} P$ provides a fundamental region for the subgroup

$$
G_{n}:=\left\langle r_{1} r_{i} \mid 2 \leq i \leq n\right\rangle
$$

\footnotetext{
${ }^{2}$ For two curves $c_{1}$ and $c_{2}$ such that $c_{2}$ begins at the final point of $c_{1}$, we denote by $c_{1} \cup c_{2}$ their concatenation.
} 
of index 2, implying that $G_{n}$ is the fundamental group of a closed orientable surface of genus $\frac{n}{2}-1$ (the polygon $P \cup r_{1} P$ has two cycles of vertices and $n-1$ pairs of edges to identify).

For odd $n$, the polygon $P \cup r_{1} P \cup r_{2} P \cup r_{2} r_{1} P$ provides a fundamental region for the group $T_{n}$ of index 4 generated by

$$
x:=r_{2} r_{1} r_{n}, \quad y:=r_{2} r_{n} r_{1}, \quad z:=r_{2} r_{1} r_{2} r_{1}, \quad x_{i}:=r_{1} r_{i}, \quad y_{i}:=r_{2} r_{1} r_{i} r_{2},
$$

where $3 \leq i \leq n-1$,

$$
T_{n}:=\left\langle x, y, z, x_{i}, y_{i} \mid 3 \leq i \leq n-1\right\rangle,
$$

implying that $T_{n}$ is the fundamental group of a closed orientable surface of genus $n-3$.

2.1.11. Toledo invariant. Following Toledo [Tol], one can introduce the Toledo invariant of a representation in PU of an arbitrary group with discrete and cocompact action on a two-disc (see also [Kre]). Every cycle of bisectors $\left(\mathrm{B}_{1}, \ldots, \mathrm{B}_{n}\right)$ defines a representation of $H_{n}$ in PU given by $r_{i} \mapsto R_{i}$. In order to calculate the Toledo invariant of this representation, we express a Kähler primitive in coordinatefree terms. In fact, such a coordinate-free approach is kind of a motto in this article. It makes it possible to significantly simplify the formulae.

We can regard every linear map $\varphi \in \operatorname{Lin}_{\mathbb{C}}(\mathbb{C} p, V)$ as a tangent vector $t_{\varphi} \in \mathrm{T}_{p} \mathbb{P}$ by defining

$$
t_{\varphi} f:=\left.\frac{d}{d \varepsilon}\right|_{\varepsilon=0} \hat{f}(p+\varepsilon \varphi(p))
$$

for a local smooth function $f$ on $\mathbb{P}$ and its lift $\hat{f}$ to $V$. In this known way, we identify the tangent space $\mathrm{T}_{p} \mathbb{P}$ with the $\mathbb{C}$-vector space $\operatorname{Lin}_{\mathbb{C}}(\mathbb{C} p, V / \mathbb{C} p)$ of linear maps. In particular, for every $p \notin \partial_{\infty} \mathbb{B}$,

$$
\mathrm{T}_{p} \mathbb{P} \simeq\langle-, p\rangle p^{\perp} .
$$

(Of course, $\langle-, p\rangle p^{\perp}$ means $\langle-, p\rangle \otimes_{\mathbb{C}} p^{\perp}$.) For a nonisotropic $p \in V$ and for $v \in p^{\perp}$, we denote

$$
v_{p}:=\langle-, p\rangle v \in \mathrm{T}_{p} \mathbb{P} .
$$

Thus, $\mathrm{T}_{p} \mathbb{P}$ is equipped with the hermitian form defined by $\left\langle v_{p}, w_{p}\right\rangle:=-\langle p, p\rangle\langle v, w\rangle$, where $v, w \in p^{\perp}$. We arrive at a positive definite hermitian metric on the complex hyperbolic plane $\mathbb{B}$ that coincides, up to the scale factor of 4 , with the one introduced in [Gol, p. 77] (see Corollary 4.1.18).

Lemma 2.1.12 [Appendix, Propositions 4.5.6 and 4.5.2]. Let $0 \neq c \in V$. Define the 1-form $P_{c}$ by the rule

$$
P_{c}\left(v_{p}\right):=-\operatorname{Im}\left(\frac{\langle p, p\rangle\langle v, c\rangle}{2\langle p, c\rangle}\right)
$$

for all $p \notin \mathbb{P} c^{\perp} \cup \partial_{\infty} \mathbb{B}$. Let $c_{1}, c_{2} \in V,\left\langle c_{1}, c_{2}\right\rangle \neq 0$. Define the function $f_{c_{1}, c_{2}}$ by the rule ${ }^{3}$

$$
f_{c_{1}, c_{2}}(p):=\frac{1}{2} \operatorname{Arg}\left(\frac{\left\langle c_{1}, p\right\rangle\left\langle p, c_{2}\right\rangle}{\left\langle c_{1}, c_{2}\right\rangle}\right)
$$

for all $p \notin \mathbb{P} c_{1}^{\perp} \cup \mathbb{P} c_{2}^{\perp}$. Then $d P_{c}=\omega$ and $P_{c_{1}}-P_{c_{2}}=d f_{c_{1}, c_{2}}$, where, for $p \in \mathbb{B}$ and $v_{p}, w_{p} \in \mathrm{T}_{p} \mathbb{B}$, the Kähler form on $\mathbb{B}$ is given by $\omega\left(v_{p}, w_{p}\right):=\operatorname{Im}\left\langle v_{p}, w_{p}\right\rangle$.

Lemma 2.1.13 [Appendix, Corollary 4.2.3]. Let $\mathrm{R}$ be an $\mathbb{R}$-plane and let $p, c \in \mathrm{R}$. Then $P_{c}\left(v_{p}\right)=0$ for every $v_{p} \in \mathrm{T}_{p} \mathrm{R}$.

\footnotetext{
${ }^{3}$ In this lemma, it is better to read Arg as a multi-valued function on $\mathbb{C} \backslash\{0\}$.
} 
Lemma 2.1.14 [Appendix, Lemma 4.1.16]. If $p, g_{1}, g_{2} \in \mathbb{B}$, then $\frac{\left\langle g_{1}, p\right\rangle\left\langle p, g_{2}\right\rangle}{\left\langle g_{1}, g_{2}\right\rangle}$ cannot be real nonnegative.

We are now able to calculate the Toledo invariant.

Proposition 2.1.15. Let $\left(\mathrm{B}_{1}, \ldots, \mathrm{B}_{n}\right)$ be a cycle of bisectors and let $\varrho: H_{n} \rightarrow$ PU be the representation given by $r_{i} \mapsto R_{i}$. Then the Toledo invariant of $\varrho$ satisfies $\tau \equiv n-\frac{\operatorname{Arg} \delta}{\pi} \bmod 2$, where $\delta$ is introduced in Definition 2.1.7.

Proof. We have $R_{i}=R\left(m_{i}\right)$, where $m_{i}$ stands for the polar point to the middle slice $M_{i}$. Assuming that $\left\langle m_{i}, m_{i}\right\rangle=1$, we obtain $R_{i}: x \mapsto 2\left\langle x, m_{i}\right\rangle m_{i}-x$ by (2.1.3). Let $b=b_{1} \cup \cdots \cup b_{n}$ be a meridional curve of $\left(\mathrm{B}_{1}, \ldots, \mathrm{B}_{n}\right)$ with vertices $s_{1}, \ldots, s_{n}$. Fixing a representative $s_{1} \in V$, define $s_{i+1}=R_{i} s_{i} \in V$. In particular, $s_{n+1}=\delta s_{1}$. Since $\left\langle R_{i} x, x\right\rangle=2\left\langle x, m_{i}\right\rangle\left\langle m_{i}, x\right\rangle-\langle x, x\rangle>0$ for every $x \in \mathbb{B}$, we obtain $\left\langle s_{i+1}, s_{i}\right\rangle>0$.

Let $D \subset \mathbb{B}$ be any disc with $\partial D=b$. In a standard way, we define a $\varrho$-equivariant continuous map $\varphi: \mathbb{H}_{\mathbb{R}}^{2} \rightarrow \mathbb{B}$ such that $\varphi(P)=D, \varphi\left(v_{i}\right)=s_{i}$, and $\varphi\left(e_{i}\right)=b_{i}$ (see 2.1.9). The Toledo invariant of $\varrho$ is defined as

$$
\tau:=\frac{4}{2 \pi} \int_{P} \varphi^{*} \omega
$$

(the hermitian metric we introduced differs by the factor of 4 from the one in [Tol] (see Corollary 4.1.18)). Taking $c \in \mathbb{B}$ and applying Lemma 2.1.12, we obtain

$$
\tau=\frac{2}{\pi} \int_{D} \omega=\frac{2}{\pi} \int_{\partial D} P_{c}=\frac{2}{\pi} \sum_{i} \int_{b_{i}} P_{c} .
$$

By Lemmas 2.1.13 and 2.1.12,

$$
\int_{b_{i}} P_{c}=\int_{b_{i}}\left(P_{c}-P_{s_{i}}\right)=\int_{b_{i}} d f_{c, s_{i}} .
$$

This number is the total variation of $\frac{1}{2} \operatorname{Arg} \frac{\langle c, p\rangle\left\langle p, s_{i}\right\rangle}{\left\langle c, s_{i}\right\rangle}$ while $p$ runs over $b_{i}$ from $s_{i}$ to $s_{i+1}$. By Lemma 2.1.14, $\frac{\langle c, p\rangle\left\langle p, s_{i}\right\rangle}{\left\langle c, s_{i}\right\rangle}$ cannot be real nonnegative. It follows that

$$
\int_{b_{i}} d f_{c, s_{i}}=\frac{1}{2} \operatorname{Arg} \frac{\left\langle c, s_{i+1}\right\rangle\left\langle s_{i+1}, s_{i}\right\rangle}{\left\langle c, s_{i}\right\rangle}-\frac{1}{2} \operatorname{Arg} \frac{\left\langle c, s_{i}\right\rangle\left\langle s_{i}, s_{i}\right\rangle}{\left\langle c, s_{i}\right\rangle}=\frac{1}{2} \operatorname{Arg} \frac{\left\langle c, s_{i+1}\right\rangle}{\left\langle c, s_{i}\right\rangle}-\frac{\pi}{2}
$$

since $\left\langle s_{i+1}, s_{i}\right\rangle>0$ and $\left\langle s_{i}, s_{i}\right\rangle<0$. Calculating $\bmod 2$, we get

$$
\begin{gathered}
\tau \equiv \frac{1}{\pi} \sum_{i}\left(\operatorname{Arg}\left\langle c, s_{i+1}\right\rangle-\operatorname{Arg}\left\langle c, s_{i}\right\rangle-\pi\right) \equiv \\
\equiv \frac{1}{\pi}\left(-n \pi+\operatorname{Arg}\left\langle c, s_{n+1}\right\rangle-\operatorname{Arg}\left\langle c, s_{1}\right\rangle\right) \equiv-n-\frac{\operatorname{Arg} \delta}{\pi} \bmod 2 .
\end{gathered}
$$

Considering the surface groups $G_{n}$ or $T_{n}$ of finite index in $H_{n}$ (see 2.1.9), we obtain the following

Corollary 2.1.17. For even $n$, the Toledo invariant of the induced representation of $G_{n}$ in PU satisfies $\tau \equiv-\frac{2 \operatorname{Arg} \delta}{\pi} \bmod 4$. For odd $n$, the Toledo invariant of the induced representation of $T_{n}$ in PU satisfies $\tau \equiv 4 n-\frac{4 \operatorname{Arg} \delta}{\pi} \bmod 8$, where $\delta$ is introduced in Definition 2.1.7. 
Proof. When passing to a finite cover, the Toledo invariant gets multiplied by the degree of the cover. In our case, this means that the first integral in Proposition 2.1.15 should be taken over two or four copies of a fundamental domain for $H_{n}$.

\subsection{Discreteness}

We intend to prove discreteness of the group $H$ generated by reflections in middle slices of a cycle of bisectors by showing that a polyhedron bounded by the cycle is fundamental. To this end, we reformulate [AGr3, Theorem 3.5] in terms of a simple transversal configuration of bisectors.

All isometries in this subsection are considered as belonging to PU.

2.2.1. Given oriented bisectors $\mathrm{B}$ and $\mathrm{B}^{\prime}$ with a common slice $S$, denote by $n$ and $n^{\prime}$ the normal vectors at $s \in S \cap \mathbb{B}$ to $\mathrm{B}$ and to $\mathrm{B}^{\prime}$, respectively. Since both $n$ and $n^{\prime}$ are tangent to the naturally oriented complex geodesic passing through $s$ and orthogonal to $S$, it makes sense to measure the oriented angle from $n$ to $n^{\prime}$. This angle is said to be the oriented angle from $\mathrm{B}$ to $\mathrm{B}^{\prime}$ at $s$.

Definition 2.2.2. A simple configuration of bisectors $\left(\mathrm{B}_{1}, \ldots, \mathrm{B}_{n}\right)$ gives rise to the closed solid torus $\partial_{0} P:=\mathrm{B}_{1} \cup \cdots \cup \mathrm{B}_{n}$ fibred by slices that divides $\overline{\mathbb{B}}$ into two closed connected parts. The part $P$ situated on the side of the normal vector to each $\mathrm{B}_{i}$ is called the polyhedron of the configuration. The torus $T:=\partial_{\infty} \partial_{0} P$ divides the boundary of $P$ into two closed parts, $\partial P=\partial_{0} P \cup \partial_{\infty} P$. So, $T=\partial \partial_{\infty} P=$ $\partial_{0} P \cap \partial_{\infty} P$. The ideal meridional curves are contained in $T$. In the particular case of a cycle of bisectors, $\partial_{0} P$ is also fibred by meridional curves (hence, $T$ is fibred by the ideal meridional curves).

2.2.3. Let $\left(\mathrm{B}_{1}, \ldots, \mathrm{B}_{n}\right)$ be a simple configuration of bisectors with common slices $S_{i}$ 's, where $\mathrm{B}_{i}:=$ $\mathrm{B}\left[S_{i}, S_{i+1}\right]$ (the indices are modulo $n$ ). A face-paring of the polyhedron $P$ of the configuration is an involution $-:\{1, \ldots, n\} \rightarrow\{1, \ldots, n\}$ and a family of isometries $I_{i} \in \mathrm{PU}$ satisfying $I_{i} \mathrm{~B}_{i}=\mathrm{B}_{\bar{i}}^{-}$and $I_{i} I_{\bar{i}}=1$ for all $i$, where $\mathrm{B}^{-}$denotes the segment $\mathrm{B}$ taken with the opposite orientation.

Define a geometric cycle of common slices as follows. Start from an ordered triple $\left(\mathrm{B}_{\bar{i}_{0}}, S_{l_{0}}, \mathrm{~B}_{i_{1}}\right)$, where $S_{l_{0}}$ is a common slice of two different segments $\mathrm{B}_{\bar{i}_{0}}, \mathrm{~B}_{i_{1}}$. (In other words, either $i_{1}=\bar{i}_{0}+1$ with $l_{0}=i_{1}$ or $\bar{i}_{0}=i_{1}+1$ with $l_{0}=\bar{i}_{0}$.) Applying $I_{i_{1}}$ to $\mathrm{B}_{i_{1}}$ and to $S_{l_{0}}$, we obtain a new triple $\left(\mathrm{B}_{\bar{i}_{1}}, S_{l_{1}}, \mathrm{~B}_{i_{2}}\right)$, where $S_{l_{1}}:=I_{i_{1}} S_{l_{0}}$ and $\mathrm{B}_{i_{2}}$ is uniquely determined by the requirement that $S_{l_{1}}$ is a common slice of two different segments $\mathrm{B}_{\bar{i}_{1}}, \mathrm{~B}_{i_{2}}$. Then we apply $I_{s_{2}}$ to $\mathrm{B}_{i_{2}}$ and to $S_{l_{1}}$, obtain $\left(\mathrm{B}_{\bar{i}_{2}}, S_{l_{2}}, \mathrm{~B}_{i_{3}}\right)$, and so on. When we arrive back at $\left(\mathrm{B}_{\bar{i}_{k}}, S_{l_{k}}, \mathrm{~B}_{i_{k+1}}\right)=\left(\mathrm{B}_{\bar{i}_{0}}, S_{l_{0}}, \mathrm{~B}_{i_{1}}\right)$ (not necessarily for the first time), we obtain a cycle of common slices and its isometry $I:=I_{i_{k}} \ldots I_{i_{1}}$. (The least possible $k$ provides a combinatorial cycle.) The cycle is geometric if its isometry $I$ is the identity and $k$ is minimal with $I=1$. In this case, $I_{i_{k}} \ldots I_{i_{1}}=1$ is the relation of the geometric cycle.

Consider a geometric cycle as above. Pick a point $s_{0} \in S_{l_{0}} \cap \mathbb{B}$, define $s_{j}:=I_{i_{j}} \ldots I_{i_{1}} s_{0} \in S_{l_{j}}$, and denote by $\alpha_{j}$ the oriented angle from $\mathrm{B}_{l_{j}}$ to $\mathrm{B}_{l_{j}-1}^{-}$at $s_{j}$. The sum $\alpha:=\alpha_{1}+\cdots+\alpha_{k}$ is the total angle of the geometric cycle at $s_{0}$.

Strong Simplicity IV [AGr3, Section 3] follows immediately from the fact that $\mathrm{B}_{i}$ and $\mathrm{B}_{j}$ are disjoint in $\overline{\mathbb{B}}$ for $j \notin\{i-1, i, i+1\}$ because this implies the metric separability of $\mathrm{B}_{i} \cap \mathbb{B}$ and $\mathrm{B}_{j} \cap \mathbb{B}$. Condition (1) of [AGr3, Theorem 3.5] (requiring that the face-pairing isometries send the interior of $P$ into the exterior of $P$ ) follows directly from $I_{i} \mathrm{~B}_{i}=\mathrm{B}_{\bar{i}}^{-}$. As in [AGr3], we denote by $N(X, \varepsilon)$ the $\varepsilon$-neighbourhood of $X \subset \mathbb{B}$. Condition (3) of $[\mathrm{AGr} 3$, Theorem 3.5] for a simple transversal configuration of bisectors is verified in the following

Lemma 2.2.4 [Appendix, Lemma 4.3.6]. Let $\mathrm{B}$ and $\mathrm{B}^{\prime}$ be full bisectors transversal along a common slice $S$. Then, for every $\vartheta>0$, there exists $\varepsilon>0$ such that $\mathrm{B}^{\prime} \cap N(\mathrm{~B} \cap \mathbb{B}, \varepsilon) \subset N(S \cap \mathbb{B}, \vartheta)$.

Now we can reformulate [AGr3, Theorem 3.5] for a simple transversal configuration of bisectors.

Theorem 2.2.5. Let $\left(\mathrm{B}_{1}, \ldots, \mathrm{B}_{n}\right)$ be a simple transversal configuration of bisectors whose polyhedron $P$ is equipped with a face-pairing such that every common slice $S_{i}$ belongs to some geometric 
cycle. Suppose that, for every geometric cycle of common slices, there exists a point in a common slice of the cycle at which the total angle of the cycle equals $2 \pi$. Then $P$ is a fundamental polyhedron for the group $G$ generated by the face-pairing isometries and the defining relations of $G$ are the relations of the geometric cycles and the relations $I_{i} I_{\bar{i}}=1$.

When dealing with a transversal cycle of bisectors, we define $\bar{i}:=i$ and $I_{i}:=R_{i}$. In this case, we obtain a unique geometric cycle.

Corollary 2.2.6. Let $\left(\mathrm{B}_{1}, \ldots, \mathrm{B}_{n}\right)$ be a simple transversal cycle of bisectors with total angle $2 \pi$ at some point and let $P$ denote the polyhedron of the cycle. Then the subgroup $H \subset \mathrm{PU}$ generated by the reflections in the middle slices of the $\mathrm{B}_{i}$ 's is discrete and $P$ is a fundamental region in $\mathbb{B}$ for $H$. The group $H$ is isomorphic to $H_{n}$.

\subsection{Simplicity and transversality}

In order to apply Corollary 2.2.6, we need a criterion for the simplicity of a cycle. We have found no adequate numerical criterion of this sort since it is rather difficult to decide whether two segments of bisectors intersect or not (see, for instance, [San]). Fortunately, by appealing to a simple kind of Poincaré duality, the simplicity of a cycle can be inferred from some extra transversalities (see Criterion 2.3.9).

All isometries in this subsection are considered as belonging to PU.

2.3.1. Notation concerning bisectors. Every two distinct points $p_{1}, p_{2} \in \overline{\mathbb{B}}$ determine a closed oriented geodesic segment $\mathrm{G}\left[p_{1}, p_{2}\right]$ from $p_{1}$ to $p_{2}$ and the corresponding closed oriented segment of bisector $\mathrm{B}\left[p_{1}, p_{2}\right]$ whose real spine is $\mathrm{G}\left[p_{1}, p_{2}\right]$. (Note that we also use to specify a closed oriented segment of bisector in terms of two ultraparallel complex geodesics as in 2.1.1.) For an oriented segment of bisector $\mathrm{B}:=\mathrm{B}\left[p_{1}, p_{2}\right]$, we denote by $\prec \mathrm{B} \succ$ the full bisector of the segment, endowed with the corresponding orientation. Hence, $\prec \mathrm{B} \succ=\mathrm{B}[u, v]$, where $u$ and $v$ are the vertices of $\prec \mathrm{B} \succ$ taken in a suitable order. We also denote $\mathrm{B} \succ:=\mathrm{B}\left[p_{1}, v\right]$ and $\mathrm{B}^{-}:=\mathrm{B}\left[p_{2}, p_{1}\right]$ (so, $\mathrm{B}^{-}$is $\mathrm{B}$ taken with the opposite orientation). In this way, we deal with three kinds of segments of bisectors: finite, semifinite, and infinite (full bisectors).

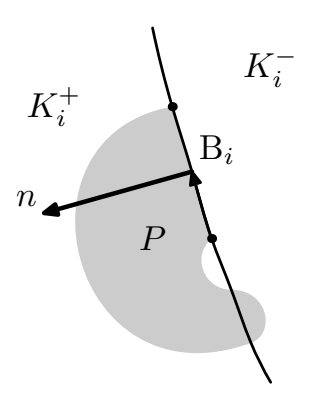

Every full oriented bisector $\mathrm{B}$ divides $\overline{\mathrm{B}}$ into two halfspaces (closed 4-balls) $K^{+}$and $K^{-}$, where $K^{+}$lies on the side of the normal vector to B. Note that, in general, the fundamental polyhedron $P$ in Corollary 2.2.6 is not contained in the half-space $K_{i}^{+}$bounded by $\prec \mathrm{B}_{i} \succ$.

Lemma 2.3.2 [Appendix, Lemma 4.3.5]. Let B and $\mathrm{B}^{\prime}$ be two full bisectors transversal along a common slice $S$. Then $\mathrm{B}, \mathrm{B}^{\prime}$, and $\partial_{\infty} \mathbb{B}$ are transversal and $\mathrm{B} \cap \mathrm{B}^{\prime}=S$.

Let $\mathrm{B}$ and $\mathrm{B}^{\prime}$ be two segments of bisectors that begin with a common slice $S$. Suppose that $\prec \mathrm{B} \succ$ and $\prec \mathrm{B}^{\prime} \succ$ are transversal along $S$. Then, by Lemma 2.3.2, $\prec \mathrm{B} \succ$ and $\prec \mathrm{B}^{\prime} \succ$ divide $\overline{\mathbb{B}}$ into four closed 4-balls. We define the sector $A$ from $\mathrm{B} \succ$ to $\mathrm{B}^{\prime} \succ$ as follows. If the oriented angle from $\mathrm{B} \succ$ to $\mathrm{B}^{\prime} \succ$ at a point in $S$ does not exceed $\pi$ (it does not matter at which point in $S$ we measure the angle), then we put $A:=K^{+} \cap K^{\prime-}$, where $K^{+}$and $K^{\prime+}$

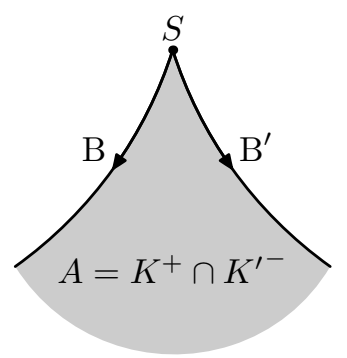
stand respectively for the half-spaces bounded by $\prec \mathrm{B} \succ$ and $\prec \mathrm{B}^{\prime} \succ$. Otherwise, we put $A:=K^{+} \cup K^{\prime-}$.

2.3.3. Transversal adjacency. Let $C, M_{1}, M_{2}$ be pairwise ultraparallel complex geodesics. We connect them by oriented segments of bisectors $\mathrm{B}_{1}:=\mathrm{B}\left[C, M_{1}\right], \mathrm{B}:=\mathrm{B}\left[M_{1}, M_{2}\right]$, and $\mathrm{B}_{2}:=\mathrm{B}\left[M_{2}, C\right]$ thus obtaining an oriented triangle of bisectors $\Delta\left(C, M_{1}, M_{2}\right)$. The triangle is said to be transversal if the corresponding full bisectors are transversal along their common slices. Since $\prec \mathrm{B}_{1} \succ$ and $\prec \mathrm{B}_{2} \succ$ are transversal along $C$, either the oriented angle from $\mathrm{B}_{1} \succ$ to $\mathrm{B}_{2}^{-} \succ$ is less than $\pi$ or the oriented angle 
from $\mathrm{B}_{2}^{-} \succ$ to $\mathrm{B}_{1} \succ$ is less than $\pi$. We denote by $D$ the sector containing the smaller angle and call it the interior sector at $C$. So, either $D=K_{1}^{+} \cap K_{2}^{+}$or $D=K_{1}^{-} \cap K_{2}^{-}$, where $K_{i}^{+}$and $K^{+}$denote the half-spaces bounded by $\prec \mathrm{B}_{i} \succ$ and $\prec \mathrm{B} \succ$. Similarly, we define the interior sector $D_{i}$ between $\mathrm{B}$ and $\mathrm{B}_{i}$ at $M_{i}$. By altering the orientation of the triangle, we can always assume that $D=K_{1}^{+} \cap K_{2}^{+}$.

Given one more complex geodesic $S$ ultraparallel to $M_{1}$ and to $M_{2}$ such that the triangle $\Delta\left(S, M_{2}, M_{1}\right)$ is also transversal, denote $\mathrm{B}_{1}^{\prime}:=\mathrm{B}\left[M_{1}, S\right]$ and $\mathrm{B}_{2}^{\prime}:=\mathrm{B}\left[S, M_{2}\right]$. We say that the triangle $\Delta\left(S, M_{2}, M_{1}\right)$ is transversally adjacent to the triangle $\Delta\left(C, M_{1}, M_{2}\right)$ if some point in $S$ belongs to $D$ and if the bisectors $\prec \mathrm{B}_{i} \succ$ and $\prec \mathrm{B}_{i}^{\prime} \succ$ are transversal along $M_{i}$ for $i=1,2$.

Lemma 2.3.4. Let $\Delta\left(C, M_{1}, M_{2}\right)$ be a transversal triangle of bisectors oriented so that $D=K_{1}^{+} \cap K_{2}^{+}$, where $D$ stands for the interior sector at $C$. Then the segment $\mathrm{B}:=\mathrm{B}\left[M_{1}, M_{2}\right]$ is contained in $D$. Moreover, for the interior sector $D_{i}$ at $M_{i}$, we have $D_{i}=K^{+} \cap K_{i}^{+}$.

Suppose that some transversal triangle $\Delta\left(S, M_{2}, M_{1}\right)$ is transversally adjacent to $\Delta\left(C, M_{1}, M_{2}\right)$. Then ${ }^{4} \Delta\left(S, M_{2}, M_{1}\right) \subset D$.

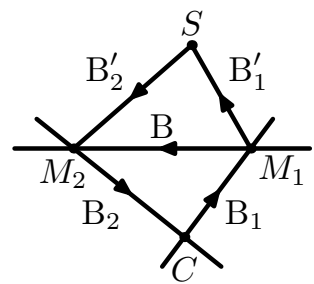

Proof. Since B is connected and intersects $\prec \mathrm{B}_{i} \succ$ only in $M_{i}$, the segment B is contained in one of the four sectors formed by the $\prec \mathrm{B}_{i} \succ$ 's. The only sector that contains both $M_{i}$ 's is $D$. This proves that $\mathrm{B} \subset D$.

Suppose that $D_{1}=K^{-} \cap K_{1}^{-}$. Then, by the above statement, we have $\mathrm{B}_{2} \subset D_{1}$, which implies $\mathrm{B}_{2} \subset K_{1}^{-}$. On the other hand, $\mathrm{B}_{2} \subset D \subset K_{1}^{+}$. A contradiction. The same works for $D_{2}$. So, $D_{i}=$ $K^{+} \cap K_{i}^{+}$.

The bisector $\prec \mathrm{B}_{1}^{\prime} \succ$ intersects $\prec \mathrm{B}_{1} \succ$ only in $M_{1}$. Hence, either $\mathrm{B}_{1}^{\prime} \succ \subset K_{1}^{+}$or $\mathrm{B}_{1}^{\prime} \succ \subset K_{1}^{-}$. Since some point of $S$ belongs to $D \subset K_{1}^{+}$and $S \subset \mathrm{B}_{1}^{\prime} \succ$, we conclude that $\mathrm{B}_{1}^{\prime} \succ \subset K_{1}^{+}$. By the same reason, $\mathrm{B}_{2}^{\prime-} \succ \subset K_{2}^{+}$. From $\mathrm{B} \subset D$, we derive that $\mathrm{B} \succ \subset K_{1}^{+}$and $\mathrm{B}^{-} \succ \subset K_{2}^{+}$. Consequently, $D_{1}^{\prime} \subset K_{1}^{+}$and $D_{2}^{\prime} \subset K_{2}^{+}$, where $D_{i}^{\prime}$ stands for the interior sector of $\Delta\left(S, M_{2}, M_{1}\right)$ at $M_{i}$. Since $\mathrm{B}_{2}^{\prime} \subset D_{1}^{\prime}$ and $\mathrm{B}_{1}^{\prime} \subset D_{2}^{\prime}$, we obtain $\mathrm{B}_{2}^{\prime} \subset K_{1}^{+}$and $\mathrm{B}_{1}^{\prime} \subset K_{2}^{+}$. Now, from $\mathrm{B}_{1}^{\prime} \succ \subset K_{1}^{+}$and $\mathrm{B}_{2}^{\prime-} \succ \subset K_{2}^{+}$, we deduce that $\mathrm{B}_{i}^{\prime} \subset D$. Therefore, $\Delta\left(S, M_{2}, M_{1}\right) \subset D$.

Definition 2.3.5. The orientation of the transversal triangle of bisectors $\Delta\left(C, M_{1}, M_{2}\right)$ dealt with in Lemma 2.3.4 is said to be counterclockwise.

Remark 2.3.6. Let $\Delta\left(C, M_{1}, M_{2}\right)$ and $\Delta\left(S, M_{2}, M_{1}\right)$ be counterclockwise-oriented transversal triangles. Suppose that $\Delta\left(S, M_{2}, M_{1}\right)$ is transversally adjacent to $\Delta\left(C, M_{1}, M_{2}\right)$. Then $\Delta\left(S, M_{2}, M_{1}\right)$ and $\Delta\left(C, M_{1}, M_{2}\right)$ lie in the distinct half-spaces bounded by $\prec \mathrm{B}\left[M_{1}, M_{2}\right] \succ$.

2.3.7. Centre of a configuration. Let $\left(\mathrm{B}_{1}, \ldots, \mathrm{B}_{n}\right)$ be a configuration of bisectors and let $C$ be a complex geodesic ultraparallel to every middle slice $M_{i}$. Denote $\mathrm{B}_{i}^{\prime}:=\mathrm{B}\left[C, M_{i}\right]$. If, for every $i$, the full bisectors $\prec \mathrm{B}_{i-1}^{\prime} \succ$ and $\prec \mathrm{B}_{i}^{\prime} \succ$ are transversal along their common slice $C$, then we call $C$ a centre of the configuration. Take $c \in C$. Denoting by $\beta_{i}$ the oriented angle from $\mathrm{B}_{i-1}^{\prime} \succ$ to $\mathrm{B}_{i}^{\prime} \succ$ at $c$, it is easy to see that $\beta:=\beta_{1}+\cdots+\beta_{n}$, the central angle of the configuration at $C$, does not depend on the choice

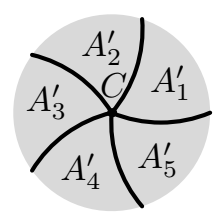
of $c \in C$ and is an integer multiple of $2 \pi$. The condition that a configuration possesses a centre does not seem too restrictive.

Lemma 2.3.8. Let $\mathrm{B}_{i}^{\prime}, 1 \leq i \leq n$, be segments of bisectors. Suppose that they all have a common slice $C$ (they all begin with $C$ ), that $\prec \mathrm{B}_{i-1}^{\prime} \succ$ and $\prec \mathrm{B}_{i}^{\prime} \succ$ are transversal along $C$ for all $i$, and that, for some $c \in C \cap \mathbb{B}$, the sum of the angles from $\mathrm{B}_{i-1}^{\prime} \succ$ to $\mathrm{B}_{i}^{\prime} \succ$ at $c$ equals $2 \pi$. Then the $\mathrm{B}_{i}^{\prime} \succ$ 's divide $\overline{\mathbb{B}}$ into $n$ sectors $A_{i}^{\prime}$ (from $\mathrm{B}_{i-1}^{\prime} \succ$ to $\mathrm{B}_{i}^{\prime} \succ$ ) such that $A_{i}^{\prime} \cap A_{j}^{\prime}=C$ if $j \notin\{i-1, i, i+1\}$ and $A_{i}^{\prime} \cap A_{i+1}^{\prime}=\mathrm{B}_{i}^{\prime} \succ$. In other words, there are no extra intersections besides the obvious ones.

${ }^{4}$ Note that $\Delta\left(S, M_{2}, M_{1}\right)$ can be 'inside' or 'outside' $\Delta\left(C, M_{1}, M_{2}\right)$ (see also Remark 2.3.6). 
Proof. Since $C \cap \mathbb{B}$ is connected, the mentioned sum of angles equals $2 \pi$ at every point in $C \cap \mathbb{B}$. Let $p \in A_{i}^{\prime} \cap A_{j}^{\prime}$ be a point that does not belong to the intersections of $A_{i}^{\prime}$ and $A_{j}^{\prime}$ listed above. Take the complex geodesic $S$ passing though $p$ and orthogonal to $C$. By Lemma 2.3.2, inside $S$, every pairwise intersection of the $\mathrm{B}_{i}^{\prime} \succ$ 's is the point $S \cap C$. Hence, inside $S$, the sectors $A_{i}^{\prime}$ 's can only intersect in the obvious way. A contradiction.

If a configuration is transversal, then $M_{i-1}$ and $M_{i}$ are ultraparallel by Lemma 2.3.2. In this case, assuming that the configuration possesses a centre $C$, we obtain the oriented triangles $\Delta_{i}:=$ $\Delta\left(C, M_{i-1}, M_{i}\right)$ and $\Delta_{i}^{\prime}:=\Delta\left(S_{i}, M_{i}, M_{i-1}\right)$.

To a certain extent, transversality implies simplicity:

Criterion 2.3.9. Let $\left(\mathrm{B}_{1}, \ldots, \mathrm{B}_{n}\right)$ be a transversal configuration of bisectors possessing a centre $C$. Suppose that the triangles $\Delta_{i}$ and $\Delta_{i}^{\prime}$ are transversal and that $\Delta_{i}^{\prime}$ is transversally adjacent to $\Delta_{i}$ for every $i$. If the central angle at some point is $2 \pi$ and the angle from $\mathrm{B}_{i-1}^{\prime} \succ$ to $\mathrm{B}_{i}^{\prime} \succ$ does not ${ }^{5}$ exceed $\pi$ for every $i$, then the cycle is simple.

Proof. By Lemma 2.3.8, the segments $\mathrm{B}_{i}^{\prime} \succ$ divide $\overline{\mathbb{B}}$ into $n$ sectors $A_{i}^{\prime}$ 's whose interiors are disjoint. The triangle $\Delta_{i}^{\prime}$ is transversally adjacent to the triangle $\Delta_{i}$, therefore, $\mathrm{B}_{i-1} \cap \mathrm{B}_{i-1}^{\prime} \succ=M_{i-1}$ and $\mathrm{B}_{i} \cap \mathrm{B}_{i}^{\prime} \succ=M_{i}$. By Lemma 2.3.4, $\mathrm{B}\left[M_{i-1}, S_{i}\right] \cup \mathrm{B}\left[S_{i}, M_{i}\right] \subset A_{i}^{\prime}$.

\subsection{Fibred polyhedra. Euler number}

Every complex hyperbolic disc bundle in the explicit series of examples we construct in Section 3 is glued from fibred polyhedra. The Euler number of the resulting bundles will be derived from 'fractional Euler quantities' of these parts.

We work in the PL category.

2.4.1. Fibred polyhedra. Let $P$ be the polyhedron of a simple configuration of bisectors (see Definition 2.2.2). In the situation described in the next lemma, $P$ is said to be fibred.

Lemma 2.4.2. Suppose that the polyhedron $P$ of a simple configuration of bisectors is a closed 4-ball, $P \simeq \mathbf{B}^{4}$. Then $P \simeq \mathbf{B}^{2} \times \mathbf{B}^{2}$ is a bundle with $\partial_{0} P \simeq \mathbb{S}^{1} \times \mathbf{B}^{2}$ being a subbundle if and only if $\partial_{\infty} P$ is a solid torus. In this case, the slice bundle structure of $\partial_{0} P$ is extendable to $P$.

Proof. The arguments are standard. If $P \simeq \mathbf{B}^{2} \times \mathbf{B}^{2}$, then $P \simeq \mathbf{B}^{4}$ and $\partial P$ is a sphere $\mathbb{S}^{3}$ decomposed into two solid tori $\partial \mathbf{B}^{2} \times \mathbf{B}^{2}$ and $\mathbf{B}^{2} \times \partial \mathbf{B}^{2}$ glued along the torus $\partial \mathbf{B}^{2} \times \partial \mathbf{B}^{2}$. Hence, $\partial_{0} P \simeq \partial \mathbf{B}^{2} \times \mathbf{B}^{2}$ implies that $\partial_{\infty} P \simeq \mathbf{B}^{2} \times \partial \mathbf{B}^{2}$.

Conversely, if $\partial_{\infty} P$ is a solid torus, then $\partial P \simeq \mathbb{S}^{3}$ is decomposed into two solid tori glued along the torus $T$. As is well known, ${ }^{6}$ such a decomposition of $\mathbb{S}^{3}$ is topologically unique. Arbitrarily fibering one of the solid tori by discs (such a fibration is isotopic to a standard one), we fiber $T$ by circles. Extending the latter circle bundle, we fiber the other solid torus by circles and obtain the compatible decompositions $T \simeq \mathbb{S}^{1} \times \mathbb{S}^{1}, \partial_{0} P \simeq \mathbb{S}^{1} \times \mathbf{B}^{2}$, and $\partial_{\infty} P \simeq \mathbf{B}^{2} \times \mathbb{S}^{1}$. Since $P \simeq \mathbf{B}^{4}$ is a cone over $\partial P \simeq \mathbb{S}^{3}$, we can readily extend these decompositions to a compatible decomposition $P \simeq \mathbf{B}^{2} \times \mathbf{B}^{2}$.

The Dehn Lemma immediately implies the

Remark 2.4.3. Let $P$ be the polyhedron of a simple configuration of bisectors. Then $P$ is fibred (hence, $P \simeq \mathbf{B}^{4}$ ) if and only if there exists some simple closed curve $c \subset T$ contractible in $\partial_{\infty} P$ such that $c$ intersects each slice of $\partial_{0} P$ exactly once, i.e., $[c]$ generates $\pi_{1}\left(\partial_{0} P\right)$.

The curve $c$ in Remark 2.4.3 is called trivializing.

\footnotetext{
${ }^{5}$ This condition is not too restrictive, since only one of these angles can exceed $\pi$.

${ }^{6}$ For example, one can use a particular case of Waldhausen's theorem [Sch].
} 
2.4.4. Gluing fibred polyhedra. Let $\left(\mathrm{B}_{1}, \ldots\right.$, $\left.\mathrm{B}_{i}, \mathrm{~B}_{i+1}, \ldots, \mathrm{B}_{n}\right), 1 \leq i<n$, and $\left(\mathrm{B}_{m}^{\prime}, \ldots, \mathrm{B}_{i+1}^{\prime}\right.$, $\left.\mathrm{B}_{i}^{-}, \ldots, \mathrm{B}_{1}^{-}\right), 1 \leq i<m$, be simple configurations of bisectors with a common sequence of bisectors (the segments common to both configurations have opposite orientations) such that the polyhedra $P_{1}$ and $P_{2}$ of the configurations intersect only in $\mathrm{B}_{1} \cup \cdots \cup \mathrm{B}_{i}$. Then we can glue $P_{1}$ and $P_{2}$ along $\mathrm{B}_{1} \cup \cdots \cup \mathrm{B}_{i}$, obtaining the gluing $P:=P_{1} \cup P_{2}$ which is the polyhedron of the simple configuration $\left(\mathrm{B}_{i+1}, \ldots, \mathrm{B}_{n}, \mathrm{~B}_{m}^{\prime}, \ldots, \mathrm{B}_{i+1}^{\prime}\right)$. The polyhedra $P$ and $R_{j} P$ in Corollary 2.2.6 yield an example of such a gluing: they are glued along $\mathrm{B}_{j}$. Suppose that $P_{1}$ and $P_{2}$ are fibred. Then the solid tori $\partial_{\infty} P_{1}$ and $\partial_{\infty} P_{2}$ intersect in the annulus $\partial_{\infty} \mathrm{B}_{1} \cup \cdots \cup$ $\partial_{\infty} \mathrm{B}_{i}$ which is an annular neighbourhood of a simple curve generating the fundamental group of each

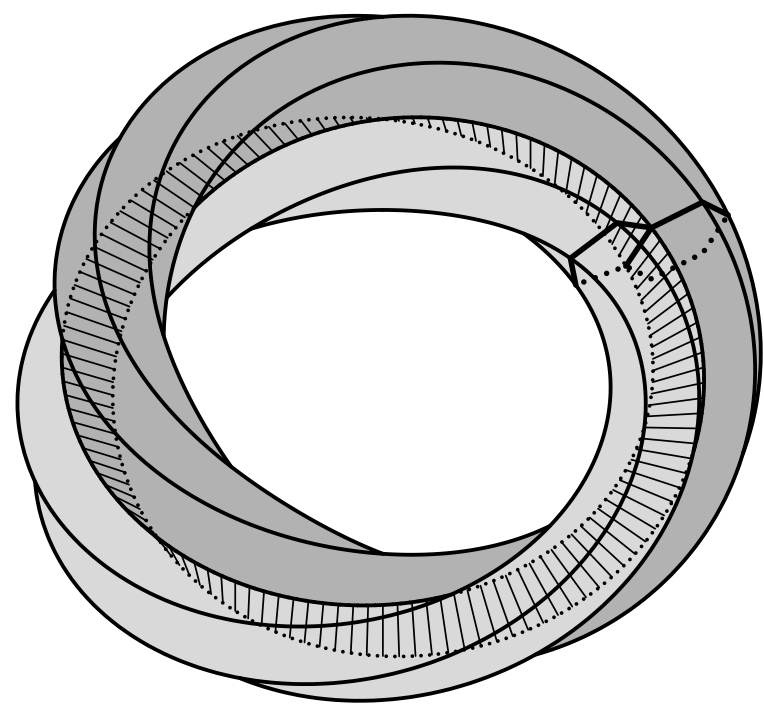
solid torus $\partial_{\infty} P_{k}, k=1,2$. Hence, we can choose a trivializing curve $c_{k}$ contractible in $\partial_{\infty} P_{k}$ so that $c_{1}$ and $c_{2}$ coincide along the annulus. Thus, we arrive at

Remark 2.4.5. Let $P_{1}$ and $P_{2}$ be fibred polyhedra and let $P:=P_{1} \cup P_{2}$ be their gluing. Then $P$ is fibred and a trivializing curve of $P$ can be obtained by gluing (and removing the common part from) trivializing curves $c_{1}$ of $P_{1}$ and $c_{2}$ of $P_{2}$ which coincide along the common sequence of bisectors.

2.4.6. Euler number. Let $P$ be a fibred polyhedron of a simple cycle, equipped with the facepairing given by reflections in middle slices, and let $b$ be a meridional curve of the cycle. Clearly, there exists a simple closed disc $D \subset P$ that intersects each fibre in $P$ exactly once and such that $b=\partial D$ and $\stackrel{\circ}{D} \subset \stackrel{\circ}{P}$ (where $\stackrel{\circ}{X}$ denotes the interior of $X$ ). We assume that meridional curves and trivializing curves are oriented with respect to the orientation of the cycle. We orient the twice fibred torus $T$ as follows: the first coordinate is the naturally oriented boundary of a slice and the second is an ideal meridional curve, already oriented. The orientation of $b$ orients $D$. We call $D$ an equivariant section of the fibred polyhedron $P$ with respect to reflections in the middle slices. For another meridional curve $b^{\prime}$ (clearly, $\left.b \cap b^{\prime}=\varnothing\right)$, we can find an equivariant section $D^{\prime}$ with $\partial D^{\prime}=b^{\prime}$ and choose $D$ and $D^{\prime}$ to be transversal. We call the number $e_{P}:=\# D \cap D^{\prime}$ (taking signs into account) the Euler number ${ }^{7}$ of the polyhedron $P$ equipped with its face-pairing (see also 2.4.8).

Clearly, $e_{P}$ measures the difference between two identifications of the slice bundle $\partial_{0} P$ with the product $\mathbb{S}^{1} \times \mathbf{B}^{2}$ : the one given by meridional curves and the one induced by the trivialization $P \simeq$ $\mathbf{B}^{2} \times \mathbf{B}^{2}$ (in the torus $T$, fibred by the boundaries of slices, the trivialization $P \simeq \mathbf{B}^{2} \times \mathbf{B}^{2}$ induces a bundle of trivializing curves). Obviously, such a difference can be measured in terms of $T$ :

Remark 2.4.7. Let $P$ be a fibred polyhedron of a simple cycle. Then $e_{P}=\# b \cap c$, where $b$ stands for an ideal meridional curve and $c$, for a trivializing curve. In other words, $[b]=e_{P} \cdot[s]$ in the group $H_{1}\left(\partial_{\infty} P, \mathbb{Z}\right)$ generated by $[s]$, where $[s]$ is represented by the boundary of a naturally oriented slice.

2.4.8. Let $P$ be a fibred polyhedron of a cycle of bisectors that satisfies the conditions of Corollary 2.2.6. Then the Euler number of the complex hyperbolic disc bundle associated to the corresponding discrete representation of $G_{n}$ or $T_{n}$ equals respectively $2 e_{P}$ or $4 e_{P}$ (see 2.1.9) since the Euler number of a polyhedron is additive with respect to gluing and the face-pairing respects the meridional curves.

\footnotetext{
${ }^{7}$ Standard arguments show that this number does not depend on the choice of the meridional curves $b$ and $b^{\prime}$ and of the equivariant sections $D$ and $D^{\prime}$.
} 
Actually, $e_{P}$ is the Euler number of the orbifold disc bundle over $\mathbb{S}^{2}(2, \ldots, 2)$ (see [BSi] for a definition). The Euler number of an orbifold disc bundle is known to be additive under finite covers.

Proposition 2.4.9. Let $\left(\mathrm{B}_{1}, \ldots, \mathrm{B}_{n}\right)$ be a simple transversal cycle of bisectors with total angle $2 \pi$ at some point and suppose that the polyhedron $P$ of the cycle is fibred. Then, for odd $n$, the complex hyperbolic manifold $\mathbb{B} / T_{n}$ is diffeomorphic to a disc bundle over a closed orientable surface of genus $n-3$ with Euler number $4 e_{P}$. For even $n$, the complex hyperbolic manifold $\mathbb{B} / G_{n}$ is diffeomorphic to a disc bundle over a closed orientable surface of genus $\frac{n}{2}-1$ with Euler number $2 e_{P}$.

\subsection{Transversal triangles}

Transversal triangles of bisectors turn out to be important geometrical objects. They can serve as building blocks for constructing (fibred) fundamental polyhedra, and not only in the way used in this paper. They are naturally equipped with an isometry of their vertices (complex geodesics), called holonomy. In this subsection, we prove that every transversal triangle provides a fibred polyhedron (Theorem 2.5.2).

2.5.1. Holonomy of a triangle. Let B be an oriented full bisector. The meridional curves induce an identification of the slices in B called the slice identification along B. By Lemma 2.1.4, for an oriented segment B $\left[S, S^{\prime}\right]$, the slice identification between $S$ and $S^{\prime}$ is induced by the reflection in the middle slice of $\mathrm{B}\left[S, S^{\prime}\right]$. Therefore, this identification is an isometry between the slices. Clearly, such an identification can also be described via a suitable one-parameter subgroup in PU.

Let $\left(\mathrm{B}_{1}, \mathrm{~B}_{2}, \mathrm{~B}_{3}\right)$ be a counterclockwise-oriented transversal triangle of bisectors (see Definition 2.3.5). Denote by $R_{i}$ the reflection in the middle slice of $\mathrm{B}_{i}$ and let $S_{1}$ stand for the initial slice of $\mathrm{B}_{1}$. We put $\varphi:=R_{3} R_{2} R_{1}$. Consider the identification of $S_{1}$ with itself given by the slice identification, first, along $\mathrm{B}_{1}$, then along $\mathrm{B}_{2}$, and, finally, along $\mathrm{B}_{3}$. This identification is obviously induced by $\varphi \in \mathrm{PU}, \varphi S_{1}=S_{1}$. We call $\varphi$ the holonomy of the triangle $\left(\mathrm{B}_{1}, \mathrm{~B}_{2}, \mathrm{~B}_{3}\right)$. The triangle is said to be elliptic, parabolic, hyperbolic, or trivial if $\varphi$, being restricted to $S_{1}$, is elliptic, parabolic, hyperbolic, or the identity.

Suppose that the triangle is elliptic. For every $s_{1} \in S_{1}$, following the counterclockwise orientation of the triangle, we can draw a meridional curve $b$ that begins at $s_{1}$. This curve ends at some $s_{1}^{\prime} \in S_{1}$. In other terms, $\varphi s_{1}=s_{1}^{\prime}$. We take $s_{1} \in \partial_{\infty} S_{1}$. Then $s_{1}^{\prime} \in \partial_{\infty} S_{1}$. Following the natural orientation of the circle $\partial_{\infty} S_{1}$, we can draw an arc $a \subset \partial_{\infty} S_{1}$ from $s_{1}^{\prime}$ to $s_{1}$ obtaining a closed oriented curve $c:=b \cup a \subset T$, where $T$ stands for the torus of the triangle. The curve $c$ is said to be standard.

In the case of a hyperbolic triangle, there are two points in $\partial_{\infty} S_{1}$ fixed by $\varphi$. They divide $\partial_{\infty} S_{1}$ into two $\varphi$-invariant parts: the R-part where $\varphi$ moves the points in the counterclockwise direction and the L-part where $\varphi$ moves the points in the clockwise direction. Let $s_{1} \in$ $\partial_{\infty} S_{1}$ be a point in the interior of the L-part. As above, we can draw a meridional curve $b$ beginning at $s_{1}$ and ending at $s_{1}^{\prime}=\varphi s_{1} \in \partial_{\infty} S_{1}$. Obviously, $s_{1}^{\prime}$ is also in the L-part. Again, we draw an arc a from

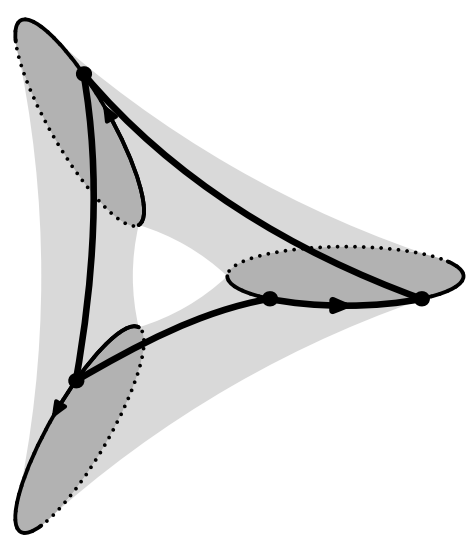
$s_{1}^{\prime}$ to $s_{1}$ in the counterclockwise direction. Clearly, $a$ is contained in the L-part of $\partial_{\infty} S_{1}$. We call the closed oriented curve $c:=b \cup a \subset T$ standard as well. Note that there are two closed meridional curves in $T$ (they correspond to the fixed points of $\varphi$ ), both isotopic to a standard one.

For a parabolic triangle, we distinguish the R-parabolic and L-parabolic cases. Exactly one point in $\partial_{\infty} S_{1}$ is fixed by $\varphi$. The isometry $\varphi$ moves all the other points in $\partial_{\infty} S_{1}$ in the same direction, counterclockwise for an R-parabolic triangle and clockwise for an L-parabolic triangle. As above, we define a standard curve for an L-parabolic triangle. In $T$, this curve is isotopic to the closed meridional curve. In the case of an R-parabolic or trivial triangle, we define no standard curve. 
We now extend the definition of the L-part of $\partial_{\infty} S_{1}$ to elliptic, parabolic, and trivial triangles. For an elliptic triangle, it is the entire $\partial_{\infty} S_{1}$. For an L-parabolic triangle, it is $\partial_{\infty} S_{1}$ without the fixed point of $\varphi$. For the other two cases, it is empty.

Counterclockwise-oriented transversal triangles of bisectors are simple configurations by Lemma 2.3.2. Hence, they fall under Definition 2.2.2. In order to prove that a polyhedron glued from transversal triangles is fibred and to be able to calculate its Euler number, we need the following

Theorem 2.5.2. Let $\left(\mathrm{B}_{1}, \mathrm{~B}_{2}, \mathrm{~B}_{3}\right)$ be a counterclockwise-oriented transversal triangle of bisectors. Then the triangle can be neither trivial nor R-parabolic. The polyhedron $P$ of the triangle is fibred and its standard curve is trivializing.

The proof of Theorem 2.5.2 is postponed until the end of this subsection.

Lemma 2.5.3 [Appendix, Lemma 4.4.18]. Let $C$ be a complex geodesic and let $\Delta_{0}:=\Delta\left(p_{1}, p_{2}, p_{3}\right)$ $\subset C$ be a counterclockwise-oriented geodesic triangle, $p_{1}, p_{2}, p_{3} \in C \cap \mathbb{B}$. Denote by $S_{1}$ the complex geodesic passing through $p_{1}$ and orthogonal to $C$ and by $R_{i}$, the reflection in the complex geodesic passing through the middle point of $\mathrm{G}\left[p_{i}, p_{i+1}\right]$ and orthogonal to $C$ (the indices are modulo 3 ). Then the isometry $\varphi:=R_{3} R_{2} R_{1}$, being restricted to $S_{1}$, is a rotation about $p_{1}$ by the angle -2 Area $\Delta_{0}$.

Lemma 2.5.4. Theorem 2.5.2 holds for every triangle of bisectors with common complex spine.

Proof. The first assertion in Theorem 2.5.2, in the case of common complex spine, follows from Lemma 2.5.3. It remains to prove the second assertion.

Every triangle of bisectors with common complex spine $C$ is built over a usual geodesic triangle $\Delta_{0}:=\Delta\left(p_{1}, p_{2}, p_{3}\right) \subset C \simeq \mathbb{H}_{\mathbb{C}}^{1}$. (The corresponding triangle of bisectors is simply the complex projective cone over $\Delta_{0}$ intersected with $\overline{\mathbb{B}}$; the vertex of the cone is the polar point to $C$.) Clearly, $\Delta_{0}$ is counterclockwise-oriented. Therefore, Area $\Delta_{0} \in\left(0, \frac{\pi}{4}\right)$ (the metric we use differs by the factor of 4 from Poincaré's one (see Corollary 4.1.18)). By Lemmas 2.1.4 and 2.5.3, the restriction of $\varphi$ to the slice $S_{1}$ is a rotation about $p_{1}$ by the angle -2 Area $\Delta_{0}$. Hence, $\ell a:=2$ Area $\Delta_{0} \in\left(0, \frac{\pi}{2}\right)$ is the angular measure (with respect to the centre $p_{1}$ ) of the arc $a$ ending at $s_{1} \in \partial_{\infty} S_{1}$. We can contract $\Delta_{0}$ to $p_{1}$ inside $\Delta_{0}$. We put $\Delta_{t}:=\Delta\left(p_{1}, p_{2}(t), p_{3}(t)\right), t \in[0,1]$, where $p_{i}(t) \in \mathrm{G}\left[p_{i}, p_{1}\right], p_{i}(0)=p_{i}$, and $p_{i}(1)=p_{1}, i=2,3$. For every $t \in[0,1]$, the triangle $\mathcal{C}_{t}$ of bisectors built over $\Delta_{t}$ contains the slice $S_{1}$. We draw the standard curve $c_{t}$ of $\mathcal{C}_{t}$ that starts with $s_{1}$. By Lemma 2.1.5, the meridional curve depends continuously on its initial point and on the bisector involved. Since $\partial_{\infty} P_{t} \subset \partial_{\infty} P_{0}$ and $\ell a_{t} \rightarrow 0$ while $t \rightarrow 1$, we can see now that $c_{0}$ contracts to $s_{1}$ inside $\partial_{\infty} P_{0}$.

2.5.5. Let $\Delta\left(S_{1}, S_{2}, S_{3}\right)$ be an oriented triangle of bisectors. Denote by $g_{i}$ the polar point to $S_{i}$, $i=1,2,3$, and define

$$
t_{i j}:=\sqrt{\operatorname{ta}\left(g_{i}, g_{j}\right)} \text { for } i \neq j, \quad \varkappa:=\frac{\left\langle g_{1}, g_{2}\right\rangle\left\langle g_{2}, g_{3}\right\rangle\left\langle g_{3}, g_{1}\right\rangle}{\left\langle g_{1}, g_{1}\right\rangle\left\langle g_{2}, g_{2}\right\rangle\left\langle g_{3}, g_{3}\right\rangle}, \quad \varepsilon:=\frac{\varkappa}{|\varkappa|}, \quad \varepsilon_{0}:=\operatorname{Re} \varepsilon, \quad \varepsilon_{1}:=\operatorname{Im} \varepsilon .
$$

The fact that the $S_{i}$ 's are ultraparallel implies $t_{i j}>1$. It follows from Sylvester's Criterion (see [KoM, p. 113]) that the determinant of the Gram matrix of $g_{1}, g_{2}, g_{3}$ is nonpositive, that is, $1+2 t_{12} t_{23} t_{31} \varepsilon_{0} \leq$ $t_{12}^{2}+t_{23}^{2}+t_{31}^{2}$, and that the equality occurs exactly when $g_{1}, g_{2}, g_{3}$ are in the same projective line. The same criterion immediately implies that the numbers $t_{i j}$ and $\varepsilon$ constitute a complete set of geometric invariants of an oriented triangle and that there exists an oriented triangle corresponding to any given values of $t_{i j}$ 's and $\varepsilon$ subject to the conditions $t_{i j}>1,|\varepsilon|=1$, and $1+2 t_{12} t_{23} t_{31} \varepsilon_{0} \leq t_{12}^{2}+t_{23}^{2}+t_{31}^{2}$.

Criterion 2.5.6 [Appendix, Criterion 4.4.2]. Suppose that $1<t_{12} \leq t_{23}, t_{31}$. Then the triangle $\Delta\left(S_{1}, S_{2}, S_{3}\right)$ is transversal if and only if $t_{12}^{2} \varepsilon_{0}^{2}+t_{23}^{2}+t_{31}^{2}<1+2 t_{12} t_{23} t_{31} \varepsilon_{0}$. A transversal triangle is counterclockwise-oriented if and only if $\varepsilon_{1}<0$. 
Lemma 2.5.7 [Appendix, Lemma 4.4.3]. The space of all counterclockwise-oriented transversal triangles of bisectors, i.e., the region in $\mathbb{R}^{4}$ given by the inequalities

$$
\left|\varepsilon_{0}\right|<1, \quad 1<t_{12} \leq t_{23}, t_{31}, \quad t_{12}^{2} \varepsilon_{0}^{2}+t_{23}^{2}+t_{31}^{2}<1+2 t_{12} t_{23} t_{31} \varepsilon_{0} \leq t_{12}^{2}+t_{23}^{2}+t_{31}^{2},
$$

is path-connected.

Lemma 2.5.8 [Appendix, Lemma 4.4.15]. A counterclockwise-oriented transversal triangle of bisectors can be neither R-parabolic nor trivial.

Proof of Theorem 2.5.2. The first assertion in the theorem corresponds to Lemma 2.5.8. We will prove the second assertion using a deformation of the parameters $\varepsilon_{0}, t_{12}, t_{23}, t_{31}$ of a given counterclockwise-oriented transversal triangle of bisectors. By Criterion 2.5.6, $\varepsilon:=\varepsilon_{0}-i \sqrt{1-\varepsilon_{0}^{2}}$. By Lemma 2.5.7, we can always reach a triangle of the type dealt with in Lemma 2.5.4. This continuous deformation of a triangle induces a continuous deformation of the holonomy $\varphi$, of the nonordered pair of the fixed points of $\varphi$ in the projective line of $S_{1}$, and, hence, of the L-part of $\partial_{\infty} S_{1}$ (nonempty by Lemma 2.5.8). Now we can choose $s_{1}$ in the L-part of $\partial_{\infty} S_{1}$ so that $s_{1}$ varies continuously during the deformation. The point $s_{1}$ generates a standard curve $c$. Since, by Lemma 2.1.5, the meridional curve depends continuously on its initial point and on the bisector involved, the curve $c$ varies continuously during the deformation.

In other words, we obtain an isotopy of a 3 -manifold $F\left(=\partial_{\infty} P\right)$ in $\mathbb{S}^{3}\left(=\partial_{\infty} \mathbb{B}\right)$ equipped with a simple curve $(=c)$ on its boundary $\left(=\partial \partial_{\infty} P\right)$ which is a simple torus $(=T)$. At the end of this isotopy, $F$ is a solid torus and the curve on its boundary is contractible in $F$. Therefore, the initial polyhedron $\partial_{\infty} P$ is a solid torus and its standard curve is contractible.

\section{Series of explicit examples}

In this section, we construct an explicit series of discrete groups with generators $U, W$ and defining relations $U^{n}=W^{n}=\left(U^{-1} W\right)^{2}=1$. Taking a subgroup of finite index, we arrive at a disc bundle over a closed orientable surface. Then we calculate the Toledo invariant and find the Euler number of these bundles. Finally, we list and discuss many particular examples.

\subsection{A couple of transversal triangles}

Following the line of Section 2, we construct two counterclockwise-oriented transversal triangles $\Delta\left(C, M_{1}, M_{2}\right)$ and $\Delta\left(S_{2}, M_{2}, M_{1}\right)$ such that $\Delta\left(S_{2}, M_{2}, M_{1}\right)$ is transversally adjacent to $\Delta\left(C, M_{1}, M_{2}\right)$. Such triangles will be used as building blocks for fundamental polyhedra. During this subsection, we elaborate some explicit and easily verifiable conditions, called the quadrangle conditions (see 3.1.17), providing the desired properties of the triangles in question (such as those listed in Proposition 3.1.18).

All isometries in this subsection are considered as belonging to SU.

3.1.1. First, we look for a regular elliptic isometry $W \in \mathrm{SU}$ (see [Gol, p. 203] for the definition) and for a reflection $R:=R(m) \in \mathrm{SU}$ in a complex geodesic $M_{1}:=\mathbb{P} m^{\perp} \cap \overline{\mathbb{B}}$ such that $U:=W R$ is a regular elliptic isometry. In what follows, the orthonormal basis $q_{1}, q_{2}, q_{3} \in V$ of signature -++ is formed by the eigenvectors of $W$. In this basis,

$$
m=\left(\begin{array}{c}
m_{1} \\
m_{2} \\
m_{3}
\end{array}\right), \quad R=\left(\begin{array}{ccc}
-2 m_{1}^{2}-1 & 2 m_{1} m_{2} & 2 m_{1} m_{3} \\
-2 m_{1} m_{2} & 2 m_{2}^{2}-1 & 2 m_{2} m_{3} \\
-2 m_{1} m_{3} & 2 m_{2} m_{3} & 2 m_{3}^{2}-1
\end{array}\right), \quad W=\left(\begin{array}{ccc}
w_{1}^{2} & 0 & 0 \\
0 & w_{2}^{2} & 0 \\
0 & 0 & w_{3}^{2}
\end{array}\right),
$$

where $m_{1}, m_{2}, m_{3}$ will be nonnegative real numbers such that $\langle m, m\rangle=1$ and the matrix form of $R$ is easily derivable from (2.1.3). We will denote by $h_{1}, h_{2}, h_{3}$ the eigenvectors of $U$ and by $u_{1}^{2}, u_{2}^{2}, u_{3}^{2}$ the corresponding eigenvalues. 
Take $k, l, n, p \in \mathbb{Z}$ subject to the conditions

$$
0 \leq k \leq l \leq n-3, \quad p=1,2
$$

and define

$$
\begin{gathered}
u_{1}:=\exp \frac{(2 n p-k) \pi i}{3 n}, \quad u_{2}:=\exp \frac{(2 n p-k-3) \pi i}{3 n}, \quad u_{3}:=\exp \frac{(2 n p+2 k+3) \pi i}{3 n}, \\
w_{1}:=\exp \frac{l \pi i}{3 n}, \quad w_{2}:=\exp \frac{(l+3) \pi i}{3 n}, \quad w_{3}:=\exp \frac{-(2 l+3) \pi i}{3 n}, \quad v:=\frac{u_{1}^{2}+u_{2}^{2}+u_{3}^{2}+w_{1}^{2}+w_{2}^{2}+w_{3}^{2}}{2} .
\end{gathered}
$$

Observe the following straightforward facts: $u_{1}^{2}, u_{2}^{2}, u_{3}^{2}$ are pairwise distinct; $w_{1}^{2}, w_{2}^{2}, w_{3}^{2}$ are pairwise distinct; $u_{1} u_{2} u_{3}=w_{1} w_{2} w_{3}=1 ; u_{1}^{2 n}=u_{2}^{2 n}=u_{3}^{2 n} ;$ and $w_{1}^{2 n}=w_{2}^{2 n}=w_{3}^{2 n}$.

The inequalities $0 \leq l \leq n-3$ imply the inequalities $0<\operatorname{Re}\left(\left(w_{1}^{2}-w_{2}^{2}\right) w_{2}\right)$ and $0<\operatorname{Re}\left(\left(w_{1}^{2}-w_{3}^{2}\right) w_{3}\right)$. Requiring that

$$
\operatorname{Re}\left(v w_{2}\right)<\operatorname{Re} w_{2}^{3}, \quad \operatorname{Re}\left(v w_{3}\right) \leq \operatorname{Re}\left(w_{1}^{2} w_{3}\right),
$$

we obtain $\operatorname{Re}\left(\left(w_{1}^{2}-w_{2}^{2}\right) w_{2}\right)<\operatorname{Re}\left(\left(w_{1}^{2}-v\right) w_{2}\right)$ and $0 \leq \operatorname{Re}\left(\left(w_{1}^{2}-v\right) w_{3}\right)$. Therefore, we can define

$$
m_{2}:=\sqrt{\frac{\operatorname{Re}\left(\left(w_{1}^{2}-v\right) w_{2}\right)}{\operatorname{Re}\left(\left(w_{1}^{2}-w_{2}^{2}\right) w_{2}\right)}}, \quad m_{3}:=\sqrt{\frac{\operatorname{Re}\left(\left(w_{1}^{2}-v\right) w_{3}\right)}{\operatorname{Re}\left(\left(w_{1}^{2}-w_{3}^{2}\right) w_{3}\right)}}, \quad m_{1}:=\sqrt{m_{2}^{2}+m_{3}^{2}-1}
$$

such that $m_{1}>0$ and $m_{2}>1$.

Lemma 3.1.4. Under conditions (3.1.2) and (3.1.3), we have $\operatorname{tr}(W R)=u_{1}^{2}+u_{2}^{2}+u_{3}^{2}$.

Proof. The equality in the lemma has the form

$$
\left(-2 m_{2}^{2}-2 m_{3}^{2}+1\right) w_{1}^{2}+\left(2 m_{2}^{2}-1\right) w_{2}^{2}+\left(2 m_{3}^{2}-1\right) w_{3}^{2}=u_{1}^{2}+u_{2}^{2}+u_{3}^{2}
$$

which is equivalent to

$$
m_{2}^{2}\left(w_{2}^{2}-w_{1}^{2}\right)+m_{3}^{2}\left(w_{3}^{2}-w_{1}^{2}\right)=v-w_{1}^{2}
$$

By definition,

$$
\begin{gathered}
m_{2}^{2}=\frac{w_{2}\left(v-w_{1}^{2}\right)+\bar{w}_{2}\left(\bar{v}-\bar{w}_{1}^{2}\right)}{w_{2}\left(w_{2}^{2}-w_{1}^{2}\right)+\bar{w}_{2}\left(\bar{w}_{2}^{2}-\bar{w}_{1}^{2}\right)}= \\
=\frac{\left(w_{2}^{2}\left(v-w_{1}^{2}\right)+\left(\bar{v}-\bar{w}_{1}^{2}\right)\right) w_{1}^{2} w_{2}^{2}}{w_{1}^{2} w_{2}^{4}\left(w_{2}^{2}-w_{1}^{2}\right)+\left(w_{1}^{2}-w_{2}^{2}\right)}=\frac{w_{1}^{2} w_{2}^{4}\left(v-w_{1}^{2}\right)+w_{1}^{2} w_{2}^{2}\left(\bar{v}-\bar{w}_{1}^{2}\right)}{\left(w_{1}^{2} w_{2}^{4}-1\right)\left(w_{2}^{2}-w_{1}^{2}\right)} .
\end{gathered}
$$

Similarly,

$$
m_{3}^{2}=\frac{w_{1}^{2} w_{3}^{4}\left(v-w_{1}^{2}\right)+w_{1}^{2} w_{3}^{2}\left(\bar{v}-\bar{w}_{1}^{2}\right)}{\left(w_{1}^{2} w_{3}^{4}-1\right)\left(w_{3}^{2}-w_{1}^{2}\right)} .
$$

It follows from $w_{1} w_{2} w_{3}=1$ that

$$
\frac{w_{1}^{2} w_{2}^{2}}{w_{1}^{2} w_{2}^{4}-1}+\frac{w_{1}^{2} w_{3}^{2}}{w_{1}^{2} w_{3}^{4}-1}=0, \quad \frac{w_{1}^{2} w_{2}^{4}}{w_{1}^{2} w_{2}^{4}-1}+\frac{w_{1}^{2} w_{3}^{4}}{w_{1}^{2} w_{3}^{4}-1}=1 .
$$

Now we can see that $\left(w_{2}^{2}-w_{1}^{2}\right) m_{2}^{2}+\left(w_{3}^{2}-w_{1}^{2}\right) m_{3}^{2}=v-w_{1}^{2}$. 
Obviously $R, W \in \mathrm{SU}$. Hence, $U:=W R \in \mathrm{SU}$. By [Gol, p. 204, Theorem 6.2.4] and Lemma 3.1.4, $U$ is a regular elliptic isometry with eigenvalues $u_{1}^{2}, u_{2}^{2}, u_{3}^{2}$. It follows from $u_{1}^{2 n}=u_{2}^{2 n}=u_{3}^{2 n}$ and $w_{1}^{2 n}=w_{2}^{2 n}=w_{3}^{2 n}$ that $U^{n}$ and $W^{n}$ belong to the centre of SU.

3.1.5. Let us find the eigenvector $h_{i}$ of $U$ corresponding to $u_{i}^{2}$. In order to exclude trivial cases, we require that

$$
u_{i}^{2}+w_{j}^{2} \neq 0 \quad \text { for all } i, j \text {. }
$$

Lemma 3.1.7. Under conditions (3.1.2), (3.1.3), and (3.1.6), $\left(\frac{\frac{m_{1}}{u_{i}^{2} w_{1}^{-2}+1}}{\frac{m_{2}^{2}}{u_{i}^{2} w_{2}^{-2}+1}} \frac{m_{3}^{3}}{u_{i}^{2} w_{3}^{-2}+1}\right)$ is the eigenvector of $U$ corresponding to $u_{i}^{2}$.

Proof. Let $x=\left(\begin{array}{l}x_{1} \\ x_{2} \\ x_{3}\end{array}\right) \neq 0$. We fix some $i=1,2,3$. The condition that $x$ is the eigenvector of $U=W R$ corresponding to $u_{i}^{2}$ is given by the equalities $2 w_{j}^{2} m_{j} f(x)=\left(u_{i}^{2}+w_{j}^{2}\right) x_{j}, j=1,2,3$, where $f(x):=-m_{1} x_{1}+m_{2} x_{2}+m_{3} x_{3}$. Thus, the inequalities (3.1.6) imply $f(x) \neq 0$ and we can take $x_{j}=\frac{m_{j}}{u_{i}^{2} w_{j}^{-2}+1}, j=1,2,3$.

3.1.8. In order to introduce the desired transversally adjacent triangles, we put

$$
h_{1}:=\left(\begin{array}{c}
\frac{m_{1}}{u_{1}^{2} w_{1}^{-2}+1} \\
\frac{m_{2}}{u_{1}^{2} w_{2}^{-2}+1} \\
\frac{m_{3}}{u_{1}^{2} w_{3}^{-2}+1}
\end{array}\right), \quad h_{2}:=\left(\begin{array}{c}
\frac{m_{1}}{u_{2}^{2} w_{1}^{-2}+1} \\
\frac{m_{2}}{u_{2}^{2} w_{2}^{-2}+1} \\
\frac{m_{3}}{u_{2}^{2} w_{3}^{-2}+1}
\end{array}\right), \quad M_{2}:=W M_{1}, \quad C:=\mathbb{P} q_{2}^{\perp} \cap \overline{\mathbb{B}}, \quad S_{2}:=\mathbb{P} h_{2}^{\perp} \cap \overline{\mathbb{B}}
$$

and require that

$$
\left\langle h_{1}, h_{1}\right\rangle<0, \quad 1<\operatorname{ta}(m, W m), \quad 1<\operatorname{ta}\left(m, h_{2}\right) .
$$

As is well known, the eigenvectors of a regular elliptic isometry are pairwise orthogonal. Hence, only one of the $h_{i}$ 's can be negative, implying that $h_{2} \notin \overline{\mathbb{B}}$. So, $S_{2}$ is a complex geodesic and $h_{1} \in S_{2}$. Obviously, $W m$ is the polar point to $M_{2}$. The last two inequalities in (3.1.9) respectively mean that the complex geodesics $M_{1}$ and $M_{2}$ are ultraparallel and that the complex geodesics $M_{1}$ and $S_{2}$ are ultraparallel [Gol, p. 100]. Since $\left\langle q_{2}, m\right\rangle=m_{2}$, we obtain $\operatorname{ta}\left(q_{2}, m\right)=m_{2}^{2}>1$ and conclude that the complex geodesics $C$ and $M_{1}$ are ultraparallel as well. Now, it follows from $W C=C$ and from $M_{2}=W M_{1}$ that $C$ and $M_{2}$ are ultraparallel. Also, from $R M_{1}=M_{1}$ and $U=W R$, we deduce that $M_{2}=W M_{1}=W R M_{1}=U M_{1}$. The facts that $M_{1}$ and $S_{2}$ are ultraparallel, that $M_{2}=U M_{1}$, and that $S_{2}=U S_{2}$ imply that $M_{2}$ and $S_{2}$ are ultraparallel. Thus, we get the triangles $\Delta\left(C, M_{1}, M_{2}\right)$ and $\Delta\left(S_{2}, M_{2}, M_{1}\right)$.

The points $q_{2}, m, h_{2}, W m$ are polar to the complex geodesics $C, M_{1}, S_{2}, M_{2}$. The equalities $M_{2}=$ $U M_{1}=W M_{1}, U S_{2}=S_{2}$, and $W C=C$ imply that $\operatorname{ta}\left(q_{2}, m\right)=\operatorname{ta}\left(W m, q_{2}\right)$ and $\operatorname{ta}\left(h_{2}, W m\right)=$ $\mathrm{ta}\left(m, h_{2}\right)$.

We introduce the invariants

$$
\begin{gathered}
t_{12}:=t_{31}:=\sqrt{\operatorname{ta}\left(q_{2}, m\right)}=\sqrt{\operatorname{ta}\left(W m, q_{2}\right)}, \quad t_{12}^{\prime}:=t_{31}^{\prime}:=\sqrt{\operatorname{ta}\left(h_{2}, W m\right)}=\sqrt{\operatorname{ta}\left(m, h_{2}\right)}, \\
t_{23}:=t_{23}^{\prime}:=\sqrt{\operatorname{ta}(m, W m)}, \\
\varkappa:=\left\langle q_{2}, m\right\rangle\langle m, W m\rangle\left\langle W m, q_{2}\right\rangle, \quad \varepsilon:=\frac{\varkappa}{|\varkappa|}, \quad \varepsilon_{0}:=\operatorname{Re} \varepsilon, \quad \varepsilon_{1}:=\operatorname{Im} \varepsilon,
\end{gathered}
$$




$$
\varkappa^{\prime}:=\left\langle h_{2}, W m\right\rangle\langle W m, m\rangle\left\langle m, h_{2}\right\rangle, \quad \varepsilon^{\prime}:=\frac{\varkappa^{\prime}}{\left|\varkappa^{\prime}\right|}, \quad \varepsilon_{0}^{\prime}:=\operatorname{Re} \varepsilon^{\prime}, \quad \varepsilon_{1}^{\prime}:=\operatorname{Im} \varepsilon^{\prime}
$$

of the oriented triangles $\Delta\left(C, M_{1}, M_{2}\right)$ and $\Delta\left(S_{2}, M_{2}, M_{1}\right)$ (see 2.5.5) and require that

$$
\begin{aligned}
& t_{12}^{2} \varepsilon_{0}^{2}+t_{23}^{2}+t_{31}^{2}<1+2 t_{12} t_{23} t_{31} \varepsilon_{0}, \quad t_{23}^{2} \varepsilon_{0}^{2}+t_{31}^{2}+t_{12}^{2}<1+2 t_{23} t_{31} t_{12} \varepsilon_{0}, \quad \varepsilon_{1}<0, \\
& t_{12}^{\prime 2} \varepsilon_{0}^{\prime 2}+t_{23}^{\prime 2}+t_{31}^{\prime 2}<1+2 t_{12}^{\prime} t_{23}^{\prime} t_{31}^{\prime} \varepsilon_{0}^{\prime}, \quad t_{23}^{\prime 2} \varepsilon_{0}^{\prime 2}+t_{31}^{\prime 2}+t_{12}^{\prime 2}<1+2 t_{23}^{\prime} t_{31}^{\prime} t_{12}^{\prime} \varepsilon_{0}^{\prime}, \quad \varepsilon_{1}^{\prime}<0 .
\end{aligned}
$$

Since $t_{12}=t_{31}$ and $t_{12}^{\prime}=t_{31}^{\prime}$, both triangles $\Delta\left(C, M_{1}, M_{2}\right)$ and $\Delta\left(S_{2}, M_{2}, M_{1}\right)$ are transversal and counterclockwise-oriented by Criterion 2.5.6.

Criterion 3.1.12 [Appendix, Criterion 4.3.3]. Let $C, C_{1}, C_{2}$ be complex geodesics and let $g, g_{1}, g_{2}$ be their polar points. Suppose that $C$ is ultraparallel to $C_{i}$ for all $i=1,2$. Then the full bisectors $\prec \mathrm{B}\left[C, C_{1}\right] \succ$ and $\prec \mathrm{B}\left[C, C_{2}\right] \succ$ are transversal along their common slice $C$ if and only if

$$
\left|\operatorname{Re} \frac{\left\langle g_{1}, g_{2}\right\rangle\langle g, g\rangle}{\left\langle g_{1}, g\right\rangle\left\langle g, g_{2}\right\rangle}-1\right|<\sqrt{1-\frac{1}{\operatorname{ta}\left(g, g_{1}\right)}} \cdot \sqrt{1-\frac{1}{\operatorname{ta}\left(g, g_{2}\right)}} .
$$

In the following lemma, we assume that Arg takes values in $[0,2 \pi)$.

Lemma 3.1.13 [Appendix, Corollary 4.3.2]. Let $\mathcal{E} \in \mathrm{SU}$ be a regular elliptic isometry and let $e_{1}, e_{2}, e_{3} \in \mathbb{P}$ be the points corresponding to the eigenvectors of $\mathcal{E}$ such that $e_{1} \in \mathbb{B}$. Denote by $E$ the complex geodesic with the polar point $e_{2}$. Let $D$ be a complex geodesic ultraparallel to $E$. Then the oriented angle from $\mathrm{B}[E, D]$ to $\mathrm{B}[E, \mathcal{E} D]$ at $e_{1} \in E$ equals $\operatorname{Arg}\left(\xi_{2} \xi_{1}^{-1}\right)$, where $\xi_{i}$ stands for the eigenvalue of $\mathcal{E}$ corresponding to $e_{i}, i=1,2$.

It follows from $M_{2}=W M_{1}, M_{1}=U^{-1} M_{2}$, and Lemma 3.1.13 that both the angle from $\mathrm{B}\left[C, M_{1}\right]$ to $\mathrm{B}\left[C, M_{2}\right]$ at $q_{1} \in C$ and the angle from $\mathrm{B}\left[S_{2}, M_{2}\right]$ to $\mathrm{B}\left[S_{2}, M_{1}\right]$ at $h_{1} \in S_{2}$ equal $\frac{2 \pi}{n}$.

We require that

$$
\left|\operatorname{Re} \frac{\left\langle h_{2}, q_{2}\right\rangle\langle m, m\rangle}{\left\langle h_{2}, m\right\rangle\left\langle m, q_{2}\right\rangle}-1\right|<\sqrt{1-\frac{1}{\operatorname{ta}\left(m, h_{2}\right)}} \cdot \sqrt{1-\frac{1}{\operatorname{ta}\left(m, q_{2}\right)}}
$$

(although it is possible to deduce (3.1.14) from (3.1.2) and (3.1.3)). By Criterion 3.1.12, the full bisectors $\prec \mathrm{B}\left[M_{1}, C\right] \succ$ and $\prec \mathrm{B}\left[M_{1}, S_{2}\right] \succ$ are transversal along their common slice $M_{1}$. It follows from $\prec \mathrm{B}\left[M_{1}, S_{2}\right] \succ=\prec \mathrm{B}\left[R S_{2}, M_{1}\right] \succ$ that the full bisectors $\prec \mathrm{B}\left[W M_{1}, W C\right] \succ$ and $\prec \mathrm{B}\left[W R S_{2}, W M_{1}\right] \succ$ are transversal along their common slice $M_{2}=W M_{1}$. Since $W C=C, W R=U$, and $U S_{2}=S_{2}$, the full bisectors $\prec \mathrm{B}\left[M_{2}, C\right] \succ$ and $\prec \mathrm{B}\left[S_{2}, M_{2}\right] \succ$ are transversal along their common slice $M_{2}$.

Lemma 3.1.15 [Appendix, Lemma 4.2.15]. Let $g_{1}, g_{2}$ be the polar points to the ultraparallel complex geodesics $C_{1}, C_{2}$. A point $x \in \mathbb{B}$ is on the side of the normal vector to the oriented bisector $\prec \mathrm{B}\left[C_{1}, C_{2}\right] \succ$ if and only if $\operatorname{Im} \frac{\left\langle g_{1}, x\right\rangle\left\langle x, g_{2}\right\rangle}{\left\langle g_{1}, g_{2}\right\rangle} \geq 0$.

We require that

$$
\operatorname{Im} \frac{\left\langle q_{2}, h_{1}\right\rangle\left\langle h_{1}, m\right\rangle}{\left\langle q_{2}, m\right\rangle} \geq 0, \quad \operatorname{Im} \frac{\left\langle W m, h_{1}\right\rangle\left\langle h_{1}, q_{2}\right\rangle}{\left\langle W m, q_{2}\right\rangle} \geq 0 .
$$

By Lemma 3.1.15, conditions (3.1.16) express the fact that $h_{1}$ belongs to the interior sector at $C$ of the triangle $\Delta\left(C, M_{1}, M_{2}\right)$. 
3.1.17. For brevity, we call conditions (3.1.2), (3.1.3), (3.1.6), (3.1.9), (3.1.10), (3.1.11), (3.1.14), (3.1.16) the quadrangle conditions. Put $s_{2}:=h_{1}$ and $q:=q_{1}$.

Proposition 3.1.18. From the quadrangle conditions, we conclude that both $U$ and $W$ are regular elliptic of order $n$ in $\mathrm{PU}$, that the counterclockwise-oriented transversal triangle $\Delta\left(S_{2}, M_{2}, M_{1}\right)$ is transversally adjacent to the counterclockwise-oriented transversal triangle $\Delta\left(C, M_{1}, M_{2}\right)$, and that both the angle from $\mathrm{B}\left[C, M_{1}\right]$ to $\mathrm{B}\left[C, M_{2}\right]$ at $q \in C$ and the angle from $\mathrm{B}\left[S_{2}, M_{2}\right]$ to $\mathrm{B}\left[S_{2}, M_{1}\right]$ at $s_{2} \in S_{2}$ equal $\frac{2 \pi}{n}$.

\subsection{Examples of disc bundles}

From the triangles $\Delta\left(C, M_{1}, M_{2}\right)$ and $\Delta\left(S_{2}, M_{2}, M_{1}\right)$ constructed in the previous subsection, we obtain a quadrangle $\mathcal{Q}$ whose polyhedron is fibred and constitutes a fundamental region for the group $K_{n}$ generated by $U, W$ with the defining relations $U^{n}=W^{n}=\left(U^{-1} W\right)^{2}=1$ in PU. Passing to a subgroup of index $n$, that is, gluing $n$ copies of the form $W^{i} \mathcal{Q}$, we arrive at a transversal simple cycle of bisectors which provides a complex hyperbolic disc bundle by Proposition 2.4.9. We calculate the Toledo invariant of the representation provided by the cycle. Using trivializing curves of the triangles, we find how to calculate the Euler number of the polyhedron of the cycle.

Actually, the quadrangle $\mathcal{Q}$ gives rise to a complex hyperbolic disc bundle over the turnover orbifold $\mathbb{S}^{2}(n, n, 2)$. One can define the (rational) Euler number of such bundles as in [BSi]. Since the turnover group $K_{n}$ possesses a discrete and cocompact action on the Poincaré disc, one can also define the Toledo invariant of representations $K_{n} \rightarrow$ PU. In our case, both numbers are easily derivable from those of the cycle glued from copies of $\mathcal{Q}$.

All isometries in this subsection are considered as belonging to SU, unless otherwise stated.

3.2.1. First, we list the facts following from the quadrangle condition 3.1.17 that are not included in Proposition 3.1.18 : The regular elliptic isometry $W$ stabilizes $C$, fixes the point $q \in C \cap \mathbb{B}$, and maps $M_{1}$ onto $M_{2}$. The regular elliptic isometry $U$ stabilizes $S_{2}$, fixes the point $s_{2} \in S_{2} \cap \mathbb{B}$, and maps $M_{1}$ onto $M_{2}$. The isometry $R:=W^{-1} U$ is the reflection in $M_{1}$. With a straightforward calculation, we obtain $W^{n} U^{-n}=\delta$, where $\delta:=\exp \frac{2(k+l+n p) \pi i}{3}$.

Note that the segment of bisector $W \mathrm{~B}\left[M_{1}, S_{2}\right]$ is a continuation of the segment $\mathrm{B}\left[S_{2}, M_{2}\right]$. Indeed, the reflection $R^{W}:=W R W^{-1}$ in $M_{2}$ maps $S_{2}$ onto $W R W^{-1} S_{2}=W U^{-1} S_{2}=W S_{2}$.

3.2.2. Discreteness of $K_{n}$. Let $\Delta\left(C, M_{1}, M_{2}\right)$ and $\Delta\left(S_{2}, M_{2}, M_{1}\right)$ be the triangles constructed in Subsection 3.1 and assume that the quadrangle conditions 3.1.17 are valid. We call the configuration

$$
\mathcal{Q}:=\left(\mathrm{B}\left[C, M_{1}\right], \mathrm{B}\left[M_{1}, S_{2}\right], \mathrm{B}\left[S_{2}, M_{2}\right], \mathrm{B}\left[M_{2}, C\right]\right)
$$

the quadrangle of bisectors. Since the transversal triangles $\Delta\left(C, M_{1}, M_{2}\right)$ and $\Delta\left(S_{2}, M_{2}, M_{1}\right)$ are transversally adjacent, the configuration $\mathcal{Q}$ is transversal. By Remark $2.3 .6, \mathcal{Q}$ is simple and the polyhedron $Q$ of $\mathcal{Q}$ is the gluing of the polyhedra of the triangles (see 2.4.4). By Theorem 2.5.2 and Remark 2.4.5, $Q$ is fibred.

Applying Theorem 2.2.5, we will show that $Q \cap \mathbb{B}$ is a fundamental polyhedron for $K_{n} \subset$ PU. Obviously, $W$ maps $\mathrm{B}\left[C, M_{1}\right]$ onto $\mathrm{B}\left[C, M_{2}\right]$ and $U$ maps $\mathrm{B}\left[S_{2}, M_{1}\right]$ onto $\mathrm{B}\left[S_{2}, M_{2}\right]$. There are three geometric cycles of edges (see 2.2.3 for the definition). The cycle of $C$ has total angle $2 \pi$ at $q \in C$ by Proposition 3.1.18. The same concerns the cycle of $S_{2}$ and $s_{2} \in S_{2}$. The geometric cycle of $M_{1}$ has length 4 due to the relation $U^{-1} W U^{-1} W=1$. In order to verify that the total angle at a point in $M_{1} \cap \mathbb{B}$ is $2 \pi$, we recall that $W$ sends $\mathrm{B}\left[C, M_{1}\right]$ onto $\mathrm{B}\left[C, M_{2}\right]$ and that $W \mathrm{~B}\left[M_{1}, S_{2}\right]$ is a continuation of $\mathrm{B}\left[S_{2}, M_{2}\right]$ (see 3.2.1). This implies that the sum of two consecutive angles at the point in question equals $\pi$ giving the total of $2 \pi$. 
3.2.3. Cycle $\mathcal{C}$, polyhedron $P$, and disc bundle $M(n, l, k, p)$. Gluing the copies $W^{i} \mathcal{Q}, i=$ $1,2, \ldots, n$, we obtain a simple transversal cycle of bisectors $\mathcal{C}:=\left(\mathrm{B}_{1}, \ldots, \mathrm{B}_{n}\right)$, where $\mathrm{B}_{i}:=\mathrm{B}\left[S_{i}, S_{i+1}\right]$ and $S_{i}:=W^{i-2} S_{2}$ (the indices are modulo $n$ ). Indeed, $S_{2}$ and $M_{2}$ are ultraparallel and the reflection in $M_{2}$ maps $S_{2}$ onto $S_{3}=W S_{2}$ by 3.2.1. Hence, $S_{2}$ and $S_{3}$ are ultraparallel and $M_{2}$ is the middle slice of $\mathrm{B}_{2}=\mathrm{B}\left[S_{2}, S_{3}\right]$. So, $R_{i}:=R^{W^{i-1}}$ is the reflection in the middle slice $M_{i}:=W^{i-2} M_{2}$ of $\mathrm{B}_{i}=W^{i-2} \mathrm{~B}_{2}$ and

$$
R_{n} \ldots R_{1}=W^{n}\left(W^{-1} R\right)^{n}=W^{n-1}\left(R W^{-1}\right)^{n} W=W^{n-1} U^{-n} W=W^{n} U^{-n}=\delta
$$

by 3.2.1. Since the triangle $\Delta\left(S_{2}, M_{2}, M_{1}\right)$ is transversal, the bisectors $\prec \mathrm{B}_{1} \succ$ and $\prec \mathrm{B}_{2} \succ$ are transversal along $S_{2}$. This implies the transversality of $\mathcal{C}$. The fact that $\Delta_{2}^{\prime}:=\Delta\left(S_{2}, M_{2}, M_{1}\right)$ is transversally adjacent to $\Delta_{2}:=\Delta\left(C, M_{1}, M_{2}\right)$ implies that $\Delta_{i}^{\prime}:=W^{i-2} \Delta_{2}^{\prime}$ is transversally adjacent to $\Delta_{i}:=W^{i-2} \Delta_{2}$ and that $C=W C$ is a centre of $\mathcal{C}$. By Proposition 3.1.18, the central angle at $q \in C$ is $2 \pi$ because $W$ fixes $q$. By Criterion 2.3.9, $\mathcal{C}$ is simple.

Denote by $P$ the polyhedron of the cycle $\mathcal{C}$. Being glued from fibred quadrangles, $P$ is fibred.

The total angle of $\mathcal{C}$ at $s_{2} \in S_{2} \cap \mathbb{B}$ equals $2 \pi$. Indeed, the oriented angle from $\mathrm{B}_{2} \succ$ to $\mathrm{B}_{1}^{-} \succ$ at $s_{2} \in S_{2}$ equals $\frac{2 \pi}{n}$ by Proposition 3.1.18. Put $s_{i}:=W^{i-2} s_{2}$. Then the oriented angle from $\mathrm{B}_{i} \succ$ to $\mathrm{B}_{i-1}^{-} \succ$ at $s_{i} \in S_{i}$ equals $\frac{2 \pi}{n}$. It remains to observe that $R_{i} s_{i}=W^{i-1} R W^{1-i} W^{i-2} s_{2}=W^{i-1} U^{-1} s_{2}=$ $W^{i-1} s_{2}=s_{i+1}$.

By Proposition 2.4.9, we arrive at the complex hyperbolic disc bundle $M(n, l, k, p)$ diffeomorphic to $\mathbb{B} / G_{n}$ if $n$ is even and to $\mathbb{B} / T_{n}$ if $n$ is odd, where $G_{n}$ or $T_{n}$ is a torsion-free subgroup of index 2 or 4 in $H_{n}$ (see 2.1.10), hence, of index $2 n$ or $4 n$ in $K_{n}$, respectively.

3.2.5. Toledo invariant of $\mathcal{C}$. As in 2.1.11 and Proposition 2.1.15, we can calculate the Toledo invariant of the representation of $H_{n}$ (see 2.1.10) defined by the cycle $\mathcal{C}$.

Proposition 3.2.6. Suppose that the quadrangle conditions 3.1 .17 are valid. Let $t$ be an integer such that $0 \leq t<3 n$ and $t \equiv 2 n p-k-l \bmod 3 n$. Then the Toledo invariant of the representation of $H_{n}$ defined by the cycle $\mathcal{C}$ equals $\frac{2}{3} t-n$.

Proof. The point $s_{2} \in S_{2}$ generates a meridional curve of $\mathcal{C}$ with vertices $s_{i+1}:=R_{i} s_{i}$, where $R_{i}:=R^{W^{i-1}}$ and $s_{n+1}=\delta s_{1}$ (see 3.2.4). Following the proof of Proposition 2.1.15, we take $c:=q$ and, using (2.1.16), obtain

$$
\tau=\frac{1}{\pi} \sum_{i=1}^{n}\left(\operatorname{Arg} \frac{\left\langle q, s_{i+1}\right\rangle}{\left\langle q, s_{i}\right\rangle}-\pi\right)
$$

We have

$$
s_{i}=R_{i-1} \ldots R_{2} s_{2}=W^{i-2}\left(R W^{-1}\right)^{i-2} s_{2}=W^{i-2} U^{2-i} s_{2}=u_{1}^{2(2-i)} W^{i-2} s_{2}
$$

because $s_{2}=h_{1}$ is an eigenvector of $U$ with eigenvalue $u_{1}^{2}$ (see 3.1.17 and 3.1.1). Since $q$ is an eigenvector of $W$ with eigenvalue $w_{1}^{2}$,

$$
\left\langle q, s_{i}\right\rangle=u_{1}^{2 i-4}\left\langle q, W^{i-2} s_{2}\right\rangle=u_{1}^{2 i-4}\left\langle W^{2-i} q, s_{2}\right\rangle=u_{1}^{2 i-4} w_{1}^{4-2 i}\left\langle q, s_{2}\right\rangle=\left(u_{1}^{2} w_{1}^{-2}\right)^{i-2}\left\langle q, s_{2}\right\rangle .
$$

Hence, $\operatorname{Arg} \frac{\left\langle q, s_{i+1}\right\rangle}{\left\langle q, s_{i}\right\rangle}=\operatorname{Arg}\left(u_{1}^{2} w_{1}^{-2}\right)=\frac{2 t \pi}{3 n}$ and $\tau=\frac{2}{3} t-n$.

3.2.7. Holonomy of the quadrangle $\mathcal{Q}$. In order to calculate the Euler number of $P$, we need to find explicitly a trivializing curve of the polyhedron $Q$. Theorem 2.5.2 provides trivializing curves of $\Delta\left(C, M_{1}, M_{2}\right)$ and $\Delta\left(S_{2}, M_{2}, M_{1}\right)$. Therefore, we only need to study the contribution coming from the mutual position of the triangles. 
Pick a generic point $z \in \partial_{\infty} C$ and introduce the following curves and points:

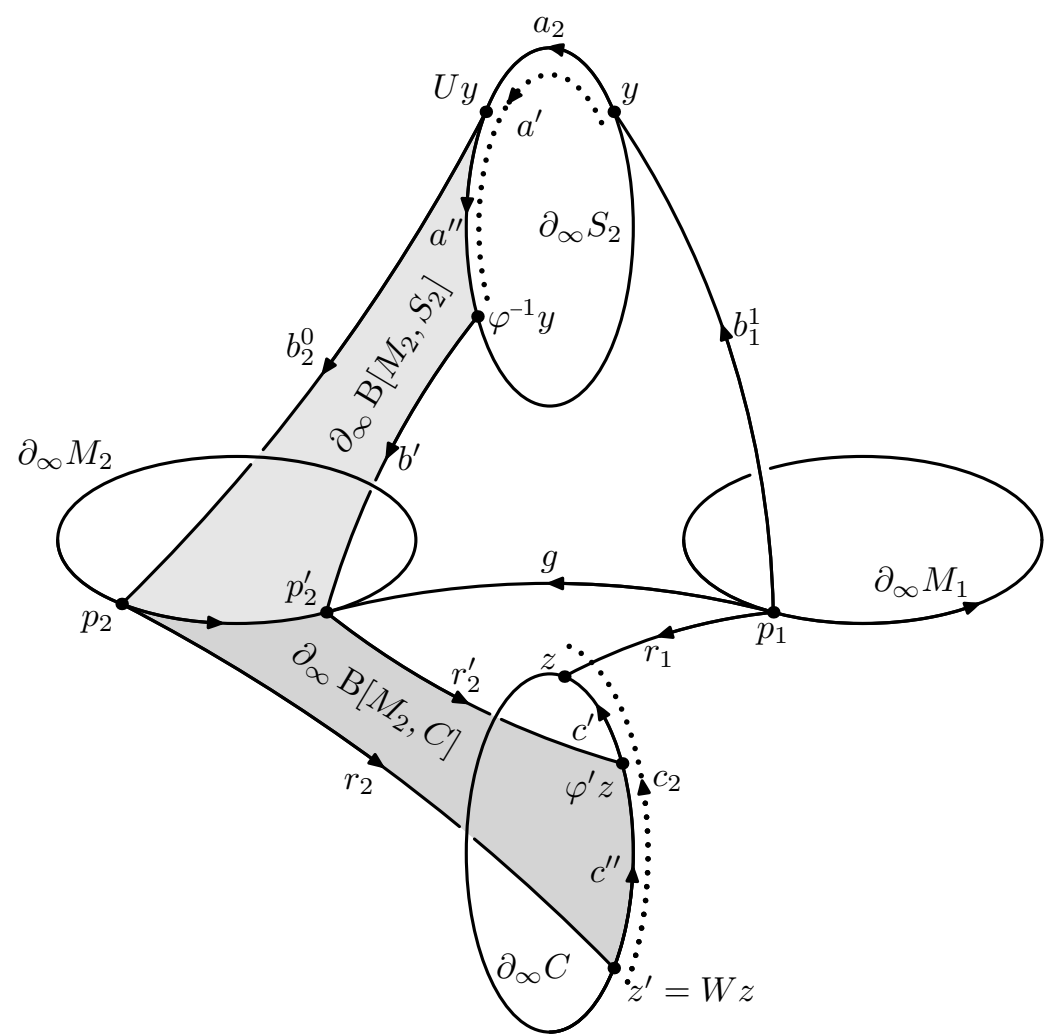

- the meridional ${ }^{8}$ curve $r_{1}^{-1} \subset \partial_{\infty} \mathrm{B}\left[C, M_{1}\right]$ that begins at $z$ and ends at $p_{1} \in \partial_{\infty} M_{1}$,

- the meridional curve $b_{1}^{1} \subset \partial_{\infty} \mathrm{B}\left[M_{1}, S_{2}\right]$ that begins at $p_{1}$ and ends at $y \in \partial_{\infty} S_{2}$,

- the naturally oriented simple arc $a_{2} \subset \partial_{\infty} S_{2}$ that begins at $y$ and ends at $U y \in \partial_{\infty} S_{2}$,

- the meridional curve $b_{2}^{0} \subset \partial_{\infty} \mathrm{B}\left[S_{2}, M_{2}\right]$ that begins at $U y$ and ends at $p_{2} \in \partial_{\infty} M_{2}$,

- the meridional curve $r_{2} \subset \partial_{\infty} \mathrm{B}\left[M_{2}, C\right]$ that begins at $p_{2}$ and ends at $z^{\prime} \in \partial_{\infty} C$,

- the naturally oriented simple arc $c_{2} \subset \partial_{\infty} C$ that begins at $z^{\prime}$ and ends at $z$.

Since $Q$ is fibred, $\partial_{\infty} Q$ is a solid torus and the group $H_{1}\left(\partial_{\infty} Q, \mathbb{Z}\right)$ is generated by $[c]$, where $c$ stands for the naturally oriented boundary of $C$. Hence, there exists $f \in \mathbb{Z}$ such that $\left[d_{2}\right]=f[c]$, where the closed curve $d_{2}$ is given by

$$
d_{2}:=r_{1}^{-1} \cup b_{1}^{1} \cup a_{2} \cup b_{2}^{0} \cup r_{2} \cup c_{2} .
$$

In order to express $f$ in terms of the holonomies $\varphi$ of $\Delta\left(S_{2}, M_{2}, M_{1}\right)$ and $\varphi^{\prime}$ of $\Delta\left(C, M_{1}, M_{2}\right)$, we introduce the following notation. Let $S$ be an oriented circle and let $t_{1}, t_{2}, t_{3} \in S$ be pairwise distinct. We define $o\left(t_{1}, t_{2}, t_{3}\right)=0$ if $t_{1}, t_{2}, t_{3}$ are in the cyclic order of $S$ and $o\left(t_{1}, t_{2}, t_{3}\right)=1$, otherwise. For an isometry $I$ of a complex geodesic $E$ and for $e \in \partial_{\infty} E$, we put $\lambda(e, I)=0$ if $e$ belongs to the L-part of $\partial_{\infty} E$ with respect to $I$ and $\lambda(e, I)=1$, otherwise.

Lemma 3.2.8. $f=\lambda(y, \varphi)+\lambda\left(z, \varphi^{\prime}\right)+o\left(y, U y, \varphi^{-1} y\right)-o\left(z, W z, \varphi^{\prime} z\right)$.

Proof. First, let us assume that $z$ belongs to the L-part of $\partial_{\infty} C$ and $y$, to the L-part of $\partial_{\infty} S_{2}$. We introduce a few more curves. The meridional curve $g \subset \partial_{\infty} \mathrm{B}\left[M_{1}, M_{2}\right]$ begins at $p_{1}$ and ends at some $p_{2}^{\prime} \in \partial_{\infty} M_{2}$. By definition, there exists a unique meridional curve $b^{\prime} \subset \partial_{\infty} \mathrm{B}\left[S_{2}, M_{2}\right]$ such that

${ }^{8}$ We denote by $x^{-1}$ the (not necessarily closed) curve $x$ taken with the opposite orientation. 
$b^{\prime} \cup g^{-1} \cup b_{1}^{1} \cup a^{\prime}$ is a trivializing curve of $\Delta\left(S_{2}, M_{2}, M_{1}\right)$, where $a^{\prime} \subset \partial_{\infty} S_{2}$ is a simple arc following the natural orientation of $\partial_{\infty} S_{2}$. Clearly, $b^{\prime}$ begins at $\varphi^{-1} y$ and ends at $p_{2}^{\prime}$. Similarly, there exists a unique meridional curve $r_{2}^{\prime} \subset \partial_{\infty} \mathrm{B}\left[M_{2}, C\right]$ such that $r_{1}^{-1} \cup g \cup r_{2}^{\prime} \cup c^{\prime}$ is a trivializing curve of $\Delta\left(C, M_{1}, M_{2}\right)$, where $c^{\prime} \subset \partial_{\infty} C$ is a simple arc drawn from $\varphi^{\prime} z$ to $z$ following the natural orientation of $\partial_{\infty} C$. So, $r_{2}^{\prime}$ ends at $\varphi^{\prime} z$.

By Remark 2.4.5, $r_{1}^{-1} \cup b_{1}^{1} \cup a^{\prime} \cup b^{\prime} \cup r_{2}^{\prime} \cup c^{\prime}$ is a trivializing curve of $Q$. In terms of 1-chains modulo boundaries, that is, in $C_{1}\left(\partial_{\infty} Q, \mathbb{Z}\right) / \partial C_{0}\left(\partial_{\infty} Q, \mathbb{Z}\right)$, this fact can be written as $-\left[r_{1}\right]+\left[b_{1}^{1}\right]+\left[a^{\prime}\right]+\left[b^{\prime}\right]+$ $\left[r_{2}^{\prime}\right]+\left[c^{\prime}\right]=0$.

Denote by $a^{\prime \prime} \subset \partial_{\infty} S_{2}$ the simple arc from $U y$ to $\varphi^{-1} y$ that follows the natural orientation of $\partial_{\infty} S_{2}$. It is easy to see that $\left[a_{2}\right]+\left[a^{\prime \prime}\right]-\left[a^{\prime}\right]=o\left(y, U y, \varphi^{-1} y\right)\left[\partial_{\infty} S_{2}\right]$ in terms of 1-chains modulo boundaries. Let $c^{\prime \prime} \subset \partial_{\infty} C$ stand for the simple arc from $z^{\prime}$ to $\varphi^{\prime} z$ that follows the natural orientation of $\partial_{\infty} C$. As above, $\left[c^{\prime \prime}\right]+\left[c^{\prime}\right]-\left[c_{2}\right]=o\left(z, z^{\prime}, \varphi^{\prime} z\right)[c]$. Since $-\left[r_{2}\right]-\left[b_{2}^{0}\right]+\left[a^{\prime \prime}\right]+\left[b^{\prime}\right]+\left[r_{2}^{\prime}\right]-\left[c^{\prime \prime}\right]=0,\left[\partial_{\infty} S_{2}\right]=[c]$, and $\left[d_{2}\right]=-\left[r_{1}\right]+\left[b_{1}^{1}\right]+\left[a_{2}\right]+\left[b_{2}^{0}\right]+\left[r_{2}\right]+\left[c_{2}\right]$, we have

$$
\begin{gathered}
{\left[d_{2}\right]=-\left[r_{1}\right]+\left[b_{1}^{1}\right]+\left[a_{2}\right]+\left[b_{2}^{0}\right]+\left[r_{2}\right]+\left[c_{2}\right]-\left[r_{2}\right]-\left[b_{2}^{0}\right]+\left[a^{\prime \prime}\right]+\left[b^{\prime}\right]+\left[r_{2}^{\prime}\right]-\left[c^{\prime \prime}\right]+} \\
+\left[r_{1}\right]-\left[b_{1}^{1}\right]-\left[a^{\prime}\right]-\left[b^{\prime}\right]-\left[r_{2}^{\prime}\right]-\left[c^{\prime}\right]=\left[a_{2}\right]+\left[a^{\prime \prime}\right]-\left[a^{\prime}\right]+\left[c_{2}\right]-\left[c^{\prime \prime}\right]-\left[c^{\prime}\right]=\left(o\left(y, U y, \varphi^{-1} y\right)-o\left(z, z^{\prime}, \varphi^{\prime} z\right)\right)[c] .
\end{gathered}
$$

We claim that $z^{\prime}=W z$. Indeed, $U$ maps $\mathrm{B}\left[S_{2}, M_{1}\right]$ onto $\mathrm{B}\left[S_{2}, M_{2}\right]$ and $y$ to $U y$. Hence, it maps $b_{1}^{1}$ onto $b_{2}^{0}$. In particular, $p_{2}=U p_{1}$. It follows from $R p_{1}=p_{1}$ and $U=W R$ that $U p_{1}=W p_{1}$. So, $p_{2}=W p_{1}$. Now, by similar arguments, $W$ maps $r_{1}$ onto $r_{2}$, implying $z^{\prime}=W z$.

When (say) $y$ belongs to the R-part of $\partial_{\infty} S_{2}$, one can readily understand that the required correction equals $\lambda(y, \varphi)$.

Clearly, the terms $\lambda(y, \varphi)$ and $\lambda\left(z, \varphi^{\prime}\right)$ vanish if the triangles $\Delta\left(S_{2}, M_{2}, M_{1}\right)$ and $\Delta\left(C, M_{1}, M_{2}\right)$ are both elliptic.

3.2.9. Euler number. In order to find the Euler number of $P$, we need a few auxiliary facts.

Put $U_{i}:=U^{W^{i-2}}$ and $s_{i}:=W^{i-2} s_{2}$. The equalities $U_{2} S_{2}=S_{2}$ and $U_{2} s_{2}=s_{2}$ imply $U_{i} S_{i}=S_{i}$ and $U_{i} s_{i}=s_{i}$ by 3.2.1.

Lemma 3.2.10. $R_{i} U_{i}^{j}=U_{i+1}^{j} R_{i}$.

Proof. It suffices to show that $R_{i} U_{i}=U_{i+1} R_{i}$. The relations $U=W R$ and $R^{2}=1$ imply the relations $R W^{-1} U=1$ and $U R=W$. Since $R_{i}=R^{W^{i-1}}$,

$$
\begin{gathered}
R_{i} U_{i}=W^{i-1} R W^{1-i} W^{i-2} U W^{2-i}=W^{i-1} R W^{-1} U W^{2-i}=W, \\
U_{i+1} R_{i}=W^{i-1} U W^{1-i} W^{i-1} R W^{1-i}=W^{i-1} U R W^{1-i}=W .
\end{gathered}
$$

Lemma 3.2.11. In the complex geodesic $C$, the isometry $W^{-1}$ is the rotation about $q \in C$ by the angle $\beta:=\frac{2(l+1) \pi}{n}$. In the complex geodesic $S_{i}$, the isometry $U_{i}$ is the rotation about $s_{i} \in S_{i}$ by the angle $\alpha:=\frac{2(k+1) \pi}{n}$.

Proof. In the basis $q, q_{2}, q_{3}$ of eigenvectors of $W$, the points in $C \cap \overline{\mathbb{B}}$ have the form $\left(\begin{array}{l}1 \\ 0 \\ z\end{array}\right)$, where $z \in \mathbb{C}$ and $|z| \leq 1$. In this way, we identify $C$ with the closed unit disc in $\mathbb{C}$ centred at 0 so that $q$ corresponds to 0 . Hence, in terms of $z, W^{-1}$ acts as the multiplication by $w_{1}^{2} w_{3}^{-2}$, implying the first assertion. The same arguments work for $U_{2}=U$ and $s_{2} \in S_{2}$, which implies the second assertion.

Definition 3.2.12. Let $a \subset \partial_{\infty} S_{i}$ be an arc (not necessarily simple) that begins at $y$ and ends at $x=U_{i}^{j} y, j \in \mathbb{Z}$. We call such an arc integer. Assign to an integer arc $a \subset \partial_{\infty} S_{i}$ its angular measure la (with respect to the centre $s_{i}$ ) by the following rules: 
- For $j=1$ and a simple integer arc $a \subset \partial_{\infty} S_{i}$ drawn in the counterclockwise direction, we put $\ell a:=\alpha$ (see Lemma 3.2.11).

- If $a \subset \partial_{\infty} S_{i}$ is integer, then $\ell a^{-1}=-\ell a$.

- If $a=a^{\prime} \cup a^{\prime \prime}$ with integer $a^{\prime}, a^{\prime \prime} \subset \partial_{\infty} S_{i}$, then $\ell a=\ell a^{\prime}+\ell a^{\prime \prime}$.

- Two integer arcs that are homotopic in $\partial_{\infty} S_{i}$ have the same angular measure.

Lemma 3.2.13. Let $a \subset \partial_{\infty} S_{i}$ be an integer arc. Then the arc $R_{i} a \subset \partial_{\infty} S_{i+1}$ is integer and $\ell R_{i} a=\ell a$.

Proof. The arc $a$ begins at $y$ and ends at $x=U_{i}^{j} y, j \in \mathbb{Z}$. Hence, the $\operatorname{arc} R_{i} a \subset \partial_{\infty} S_{i+1}$ begins at $R_{i} y$ and ends at $R_{i} x=R_{i} U_{i}^{j} y=U_{i+1}^{j} R_{i} y$ by Lemma 3.2.10. It remains to observe that the fact is valid for $j=1$ and any simple integer arc $a$ drawn in the counterclockwise direction.

Proposition 3.2.14. Under the quadrangle conditions 3.1.17, the Euler number of $P$ equals $n f-$ $k-l-2$, where $f$ is calculated in Lemma 3.2.8.

Proof. We introduce the following curves and points (see also 3.2.7) :

$$
\begin{array}{rlrl}
a_{i}:=W^{i-2} a_{2} \subset \partial_{\infty} S_{i}, & b_{i}^{0}:=W^{i-2} b_{2}^{0} \subset \partial_{\infty} \mathrm{B}\left[S_{i}, M_{i}\right], & & b_{i}^{1}:=W^{i-1} b_{1}^{1} \subset \partial_{\infty} \mathrm{B}\left[M_{i}, S_{i+1}\right], \\
c_{i}:=W^{i-2} c_{2} \subset \partial_{\infty} C, & d_{i}:=W^{i-2} d_{2} \subset W^{i-2} \partial_{\infty} Q, & r_{i}:=W^{i-1} r_{1} \subset \partial_{\infty} \mathrm{B}\left[M_{i}, C\right] .
\end{array}
$$

The facts that $W p_{1}=p_{2}, W \mathrm{~B}\left[M_{1}, C\right]=\mathrm{B}\left[M_{2}, C\right]$, and $r_{2}$ is the meridional curve of $\mathrm{B}\left[M_{2}, C\right]$ generated by $p_{2}$ imply that $W r_{1}=r_{2}$. So, the $r_{i}$ 's are well defined. For the same reason, we can define $p_{i}=$ $W^{i-1} p_{1} \in \partial_{\infty} M_{i}$. Note that $b_{i}^{0}$ ends at $p_{i}$ and $b_{i}^{1}$ begins at $p_{i}$. Since $b_{i}^{0} \subset \mathrm{B}\left[S_{i}, M_{i}\right]$ and $b_{i}^{1} \subset \mathrm{B}\left[M_{i}, S_{i+1}\right]$ are meridional curves, they form a meridional curve $b_{i}:=b_{i}^{0} \cup b_{i}^{1} \subset \mathrm{B}\left[S_{i}, S_{i+1}\right]$. We obtain a closed curve

$$
\gamma:=a_{1} \cup b_{1} \cup \cdots \cup a_{n} \cup b_{n} \subset T,
$$

where $T:=\partial \partial_{\infty} P$ is the torus of $P$ (see Definition 2.2.2).

Since $P$ is fibred, $\partial_{\infty} P$ is a solid torus and the group $H_{1}\left(\partial_{\infty} P, \mathbb{Z}\right)$ is generated by $[s]$, where $s$ stands for the naturally oriented boundary of an arbitrary slice of $\mathcal{C}$. Note also that $[s]=[c]$.

The fact that $W P=P$ and $W C=C$ implies $W[c]=[c]$. Therefore, $\left[d_{1} \cup \cdots \cup d_{n}\right]=\left[d_{1}\right]+\cdots+\left[d_{n}\right]=$ $n f[c]$. On the other hand, $\left[d_{1} \cup \cdots \cup d_{n}\right]=[\gamma]+\left[c_{1} \cup \cdots \cup c_{n}\right]$ and $\left[c_{1} \cup \cdots \cup c_{n}\right]=(l+1)[c]$ by Lemma 3.2.11 because the angular measure of every $c_{i}$ with respect to the centre $q$ equals $\beta$. Thus, $[\gamma]=(n f-l-1)[c]$.

Denote by $b$ the meridional curve of $\mathcal{C}$ (see 2.1.8) that includes $b_{1}$. We have $b=b_{1}^{\prime} \cup \cdots \cup b_{n}^{\prime}$, where $b_{i}^{\prime}$ is a meridional curve of $\mathrm{B}\left[S_{i}, S_{i+1}\right]$ and $b_{1}^{\prime}=b_{1}$. Let $a_{1}^{\prime} \subset \partial_{\infty} S_{1}$ be the initial point of $b_{1}$ and, by induction, let $a_{i+1}^{\prime}=R_{i} a_{i}^{\prime} \cup a_{i+1}$ for $i=1, \ldots, n$. Using Lemma 2.1.4, it is easy to see that the curves $a_{i}^{\prime} \cup b_{i}$ and $b_{i}^{\prime} \cup R_{i} a_{i}^{\prime}$ are well defined and homotopic in the cylinder $\partial_{\infty} \mathrm{B}\left[S_{i}, S_{i+1}\right]$. In terms of 1chains modulo boundaries in the solid torus $\partial_{\infty} P$, this implies that $\left[a_{i}^{\prime}\right]+\left[b_{i}\right]=\left[b_{i}^{\prime}\right]+\left[R_{i} a_{i}^{\prime}\right]$ for all $i$. Lemma 3.2.13 provides the equality $\ell a_{i}^{\prime}=(i-1) \alpha$ (see also Lemma 3.2.11). Indeed, since $W=U R$ and $a_{i+1}=W a_{i}$, it follows that $W=U_{i+1} R_{i}$ and $a_{i+1}=U_{i+1} R_{i} a_{i}$. The equality $\ell a_{i}=\alpha$ follows

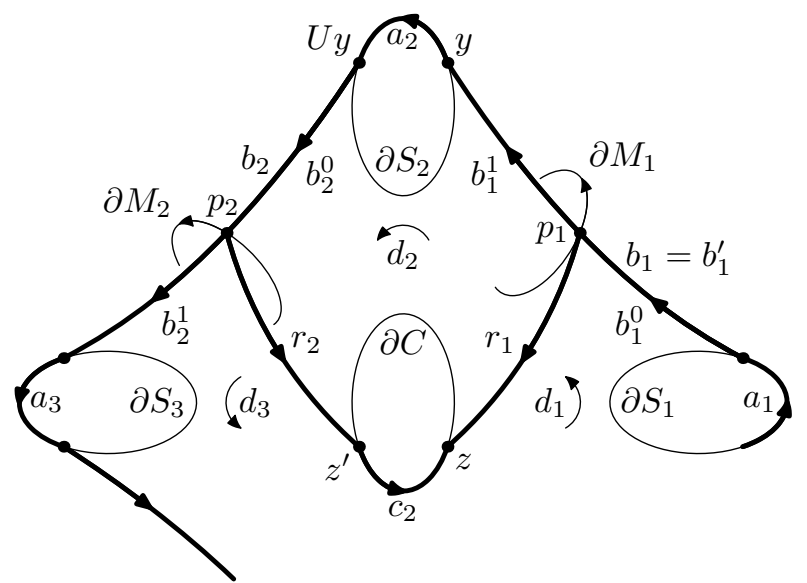
from the fact that $R_{i}$ and $U_{i+1}$ preserve the corresponding angular measures.

By induction, $\gamma$ is homotopic in $T$ to $b \cup a_{n+1}^{\prime}$. Hence, $(n f-l-1)[c]=[\gamma]=[b]+(k+1)[c]=$ $\left(e_{P}+k+1\right)[c]$. 
3.2.15. Propositions $2.4 .9,3.2 .14$, and 3.2 .6 have the following

Corollary 3.2.16. Under the quadrangle conditions 3.1.17, the Euler number $e$ of the disc bundle $M(n, l, k, p)$ over an orientable closed surface $\Sigma$ equals $4(n f-k-l-2)$ if $n$ is odd and $2(n f-k-l-2)$ if $n$ is even. Moreover, $2(\chi+e) \equiv 3 \tau \bmod 8 n$ for odd $n$ and $2(\chi+e) \equiv 3 \tau \bmod 4 n$ for even $n$, where $\chi$ is the Euler characteristic of $\Sigma$ and $\tau$ stands for the Toledo invariant of the representation of $\pi_{1} \Sigma$ associated to $M(n, l, k, p)$.

Remark 3.2.17. The rational Euler number of the disc bundle over the turnover orbifold $\mathbb{S}^{2}(n, n, 2)$ provided by the quadrangle equals $f-\frac{k+l+2}{n}$. The corresponding Toledo invariant is $\frac{2 t}{3 n}-1$ (see Proposition 3.2.6 for the definition of $t$ ).

\subsection{Some interesting examples.}

In this subsection, we list some explicit examples of complex hyperbolic disc bundles of the type $M(n, l, k, p)$ obtained with straightforward computer calculations. All we need is to find parameters $n, l, k, p$ satisfying the quadrangle conditions 3.1.17, a task easily achievable with the help of any computational tool. Our particular program has a wide margin of error, thus guaranteeing the validity of our results in the sense that we definitely prefer to lose an existing example rather than to get a doubtful one. In what follows, $e, g, \chi$, and $\tau$ stand respectively for the Euler number of the disc bundle $M(n, l, k, p) \rightarrow \Sigma$, the genus of $\Sigma$, the Euler characteristic of $\Sigma$, and the Toledo invariant of the corresponding representation $\pi_{1} \Sigma \rightarrow$ PU.

We have tested all $n \leq 1001$ and, for every $n$, all possible values of $l, k, p$. The $\mathbb{C}$-Fuchsian examples have been discarded by a direct use of Toledo's rigidity theorem [Tol] (see the beginning of Introduction). The results are as follows:

- There is no example for $n<9$ or for $n=11,12$. For any other $n \leq 1001$, there exists at least one example. The total number of examples is 308359. There are exactly 89546 examples with integer Toledo invariant. So, we obtain the first examples of discrete and faithful representations $\pi_{1} \Sigma \rightarrow$ PU with fractional Toledo invariant.

- For every example, both triangles $\Delta\left(C, M_{1}, M_{2}\right)$ and $\Delta\left(S_{2}, M_{2}, M_{1}\right)$ are elliptic, $o\left(z, W z, \varphi^{\prime} z\right)=0$, $f=o\left(y, U y, \varphi^{-1} y\right)$, and $p+f=2$. The last equality can be seen as some tiny evidence supporting the complex hyperbolic variant of the GLT-conjecture (see below).

- For every $\Sigma$ with $\chi \Sigma<0$ and for an arbitrary even integer $\tau$ subject to the Toledo necessary condition $|\tau| \leq|\chi|$, a complex hyperbolic disc bundle was constructed in [GKL]. Therefore, each of our examples with integer $\tau$ provides a couple of nonhomeomorphic complex hyperbolic disc bundles over the same $\Sigma$ and with the same $\tau$. This implies that there exist discrete and faithful representations $\pi_{1} \Sigma \rightarrow \mathrm{PU}(2,1)$ that lie in the same connected component of the space of representations [Xia] but not in the same connected component of the space of discrete and faithful representations.

- Every example satisfies the inequalities $\tau<0$ and $\frac{1}{2} \chi<e<0$. All previously known examples, including those constructed in [GKL], satisfy the inequalities $\chi \leq e \leq \frac{1}{2} \chi$.

- Every example satisfies the equality $2(\chi+e)=3 \tau$. This equality is a necessary condition for the existence of a holomorphic section of the bundle (in the sense that there exist a disc bundle structure on $M$ and a smooth holomorphic surface $\Sigma \subset M$ that intersects every fibre exactly once). It suggests the following conjecture: Every (or at least one) $M(n, l, k, p)$ possesses a disc bundle structure admitting a holomorphic section.

- The following table contains all examples with extreme values of $g, e$, and $e / \chi$ : 


\begin{tabular}{|l|r|r|r|r|r|}
\hline \multicolumn{1}{|c|}{ Manifold } & $g$ & \multicolumn{1}{c|}{$\chi$} & $e$ & \multicolumn{1}{c|}{$\tau$} & Comment \\
\hline$M(10,6,3,1)$ & 4 & -6 & -2 & $-5 \frac{1}{3}$ & minimal $g$, maximal $e$ \\
\hline$M(9,4,4,1)$ & 6 & -10 & -4 & $-9 \frac{1}{3}$ & next to minimal $g$, next to maximal $e$ \\
\hline$M(9,5,3,1)$ & 6 & -10 & -4 & $-9 \frac{1}{3}$ & next to minimal $g$, next to maximal $e$ \\
\hline$M(9,6,2,1)$ & 6 & -10 & -4 & $-9 \frac{1}{3}$ & next to minimal $g$, next to maximal $e$ \\
\hline$M(14,7,7,1)$ & 6 & -10 & -4 & $-9 \frac{1}{3}$ & next to minimal $g$, next to maximal $e$ \\
\hline$M(14,8,6,1)$ & 6 & -10 & -4 & $-9 \frac{1}{3}$ & next to minimal $g$, next to maximal $e$ \\
\hline$M(14,9,5,1)$ & 6 & -10 & -4 & $-9 \frac{1}{3}$ & next to minimal $g$, next to maximal $e$ \\
\hline$M(14,10,4,1)$ & 6 & -10 & -4 & $-9 \frac{1}{3}$ & next to minimal $g$, next to maximal $e$ \\
\hline$M(14,11,3,1)$ & 6 & -10 & -4 & $-9 \frac{1}{3}$ & next to minimal $g$, next to maximal $e$ \\
\hline$M(14,0,0,2)$ & 6 & -10 & -4 & $-9 \frac{1}{3}$ & next to minimal $g$, next to maximal $e$ \\
\hline$M(16,0,0,2)$ & 7 & -12 & -4 & $-10 \frac{2}{3}$ & next to maximal $e$ \\
\hline$M(18,0,0,2)$ & 8 & -14 & -4 & -12 & next to maximal $e$ \\
\hline$M(20,0,0,2)$ & 9 & -16 & -4 & $-13 \frac{1}{3}$ & next to maximal $e$ \\
\hline$M(22,0,0,2)$ & 10 & -18 & -4 & $-14 \frac{2}{3}$ & next to maximal $e$ \\
\hline$M(24,0,0,2)$ & 11 & -20 & -4 & -16 & next to maximal $e$ \\
\hline$M(26,0,0,2)$ & 12 & -22 & -4 & $-17 \frac{1}{3}$ & next to maximal $e$ \\
\hline$M(28,0,0,2)$ & 13 & -24 & -4 & $-18 \frac{2}{3}$ & minimal $e / \chi=\frac{1}{6}$, next to maximal $e$ \\
\hline
\end{tabular}

In principle, some examples in the above table could be isometric, for instance, those with $n=14$. However, this is not the case as it is easy to show that these bundles provide distinct points in the space $\mathcal{T}_{n}$ of discrete and faithful representations $\varrho: \pi_{1} \Sigma \rightarrow$ PU modulo conjugation by PU. Note that all examples with $n=14$ have the same known discrete invariants. We believe that those with $p=1$ are in the same connected component of $\mathcal{T}_{14}$. Moreover, we expect that there exist new Toledo-like discrete invariants that distinguish the cases $p=1$ and $p=2$ thus showing that the corresponding manifolds provide points in different connected components of $\mathcal{T}_{14}$. Such a behaviour should not be specific for $n=14$.

The fact that the congruences in Corollary 3.2.16 turn out to be equalities in our explicit examples is a sort of evidence in support of the complex hyperbolic GLT-conjecture analogous to the real hyperbolic one stated in [GLT] : A disc bundle over a closed orientable surface admits a complex hyperbolic structure if and only if $|e| \leq|\chi|$ and $\chi<0$.

- The two tables below display all 55 examples that satisfy the inequality $\frac{1}{3} \chi \leq e$ and, therefore, according to [Kui], admit a real hyperbolic structure: 


\begin{tabular}{|c|r|r|r|r|}
\hline Manifold & $g$ & \multicolumn{1}{c|}{$\chi$} & $e$ & $\tau$ \\
\hline$M(10,6,3,1)$ & 4 & -6 & -2 & $-5 \frac{1}{3}$ \\
\hline$M(16,0,0,2)$ & 7 & -12 & -4 & $-10 \frac{2}{3}$ \\
\hline$M(18,0,0,2)$ & 8 & -14 & -4 & -12 \\
\hline$M(20,0,0,2)$ & 9 & -16 & -4 & $-13 \frac{1}{3}$ \\
\hline$M(22,0,0,2)$ & 10 & -18 & -4 & $-14 \frac{2}{3}$ \\
\hline$M(22,1,0,2)$ & 10 & -18 & -6 & -16 \\
\hline$M(24,0,0,2)$ & 11 & -20 & -4 & -16 \\
\hline$M(24,1,0,2)$ & 11 & -20 & -6 & $-17 \frac{1}{3}$ \\
\hline$M(26,0,0,2)$ & 12 & -22 & -4 & $-17 \frac{1}{3}$ \\
\hline$M(26,1,0,2)$ & 12 & -22 & -6 & $-18 \frac{2}{3}$ \\
\hline$M(28,0,0,2)$ & 13 & -24 & -4 & $-18 \frac{2}{3}$ \\
\hline$M(28,1,0,2)$ & 13 & -24 & -6 & -20 \\
\hline$M(28,1,1,2)$ & 13 & -24 & -8 & $-21 \frac{1}{3}$ \\
\hline$M(28,2,0,2)$ & 13 & -24 & -8 & $-21 \frac{1}{3}$ \\
\hline$M(17,0,0,2)$ & 14 & -26 & -8 & $-22 \frac{2}{3}$ \\
\hline$M(30,1,1,2)$ & 14 & -26 & -8 & $-22 \frac{2}{3}$ \\
\hline$M(30,2,0,2)$ & 14 & -26 & -8 & $-22 \frac{2}{3}$ \\
\hline$M(32,1,1,2)$ & 15 & -28 & -8 & -24 \\
\hline$M(32,2,0,2)$ & 15 & -28 & -8 & -24 \\
\hline$M(19,0,0,2)$ & 16 & -30 & -8 & $-25 \frac{1}{3}$ \\
\hline$M(34,1,1,2)$ & 16 & -30 & -8 & $-25 \frac{1}{3}$ \\
\hline$M(34,2,1,2)$ & 16 & -30 & -10 & $-26 \frac{2}{3}$ \\
\hline$M(34,3,0,2)$ & 16 & -30 & -10 & $-26 \frac{2}{3}$ \\
\hline$M(36,1,1,2)$ & 17 & -32 & -8 & $-26 \frac{2}{3}$ \\
\hline$M(36,2,1,2)$ & 17 & -32 & -10 & -28 \\
\hline$M(21,0,0,2)$ & 18 & -34 & -8 & -28 \\
\hline$M(38,2,1,2)$ & 18 & -34 & -10 & $-29 \frac{1}{3}$ \\
\hline$M(40,2,2,2)$ & 19 & -36 & -12 & -32 \\
\hline
\end{tabular}

\begin{tabular}{|c|c|c|r|c|}
\hline Manifold & $g$ & $\chi$ & $e$ & $\tau$ \\
\hline$M(40,3,1,2)$ & 19 & -36 & -12 & -32 \\
\hline$M(23,0,0,2)$ & 20 & -38 & -8 & $-30 \frac{2}{3}$ \\
\hline$M(23,1,0,2)$ & 20 & -38 & -12 & $-33 \frac{1}{3}$ \\
\hline$M(42,2,2,2)$ & 20 & -38 & -12 & $-33 \frac{1}{3}$ \\
\hline$M(44,2,2,2)$ & 21 & -40 & -12 & $-34 \frac{2}{3}$ \\
\hline$M(25,0,0,2)$ & 22 & -42 & -8 & $-33 \frac{1}{3}$ \\
\hline$M(25,1,0,2)$ & 22 & -42 & -12 & -36 \\
\hline$M(46,3,2,2)$ & 22 & -42 & -14 & $-37 \frac{1}{3}$ \\
\hline$M(27,0,0,2)$ & 24 & -46 & -8 & -36 \\
\hline$M(27,1,0,2)$ & 24 & -46 & -12 & $-38 \frac{2}{3}$ \\
\hline$M(52,3,3,2)$ & 25 & -48 & -16 & $-42 \frac{2}{3}$ \\
\hline$M(29,1,0,2)$ & 26 & -50 & -12 & $-41 \frac{1}{3}$ \\
\hline$M(29,1,1,2)$ & 26 & -50 & -16 & -44 \\
\hline$M(29,2,0,2)$ & 26 & -50 & -16 & -44 \\
\hline$M(31,1,1,2)$ & 28 & -54 & -16 & $-46 \frac{2}{3}$ \\
\hline$M(31,2,0,2)$ & 28 & -54 & -16 & $-46 \frac{2}{3}$ \\
\hline$M(33,1,1,2)$ & 30 & -58 & -16 & $-49 \frac{1}{3}$ \\
\hline$M(35,1,1,2)$ & 32 & -62 & -16 & -52 \\
\hline$M(35,2,1,2)$ & 32 & -62 & -20 & $-54 \frac{2}{3}$ \\
\hline$M(37,1,1,2)$ & 34 & -66 & -16 & $-54 \frac{2}{3}$ \\
\hline$M(37,2,1,2)$ & 34 & -66 & -20 & $-57 \frac{1}{3}$ \\
\hline$M(41,2,2,2)$ & 38 & -74 & -24 & $-65 \frac{1}{3}$ \\
\hline$M(41,3,1,2)$ & 38 & -74 & -24 & $-65 \frac{1}{3}$ \\
\hline$M(43,2,2,2)$ & 40 & -78 & -24 & -68 \\
\hline$M(45,2,2,2)$ & 42 & -82 & -24 & $-70 \frac{2}{3}$ \\
\hline$M(47,3,2,2)$ & 44 & -86 & -28 & -76 \\
\hline$M(53,3,3,2)$ & 50 & -98 & -32 & $-86 \frac{2}{3}$ \\
\hline
\end{tabular}

- The following tables show all examples for $n=101(g=98, \chi=-194)$. They illustrate a typical behaviour of $l, k, p$, and the Euler number for some fixed $n$ : 


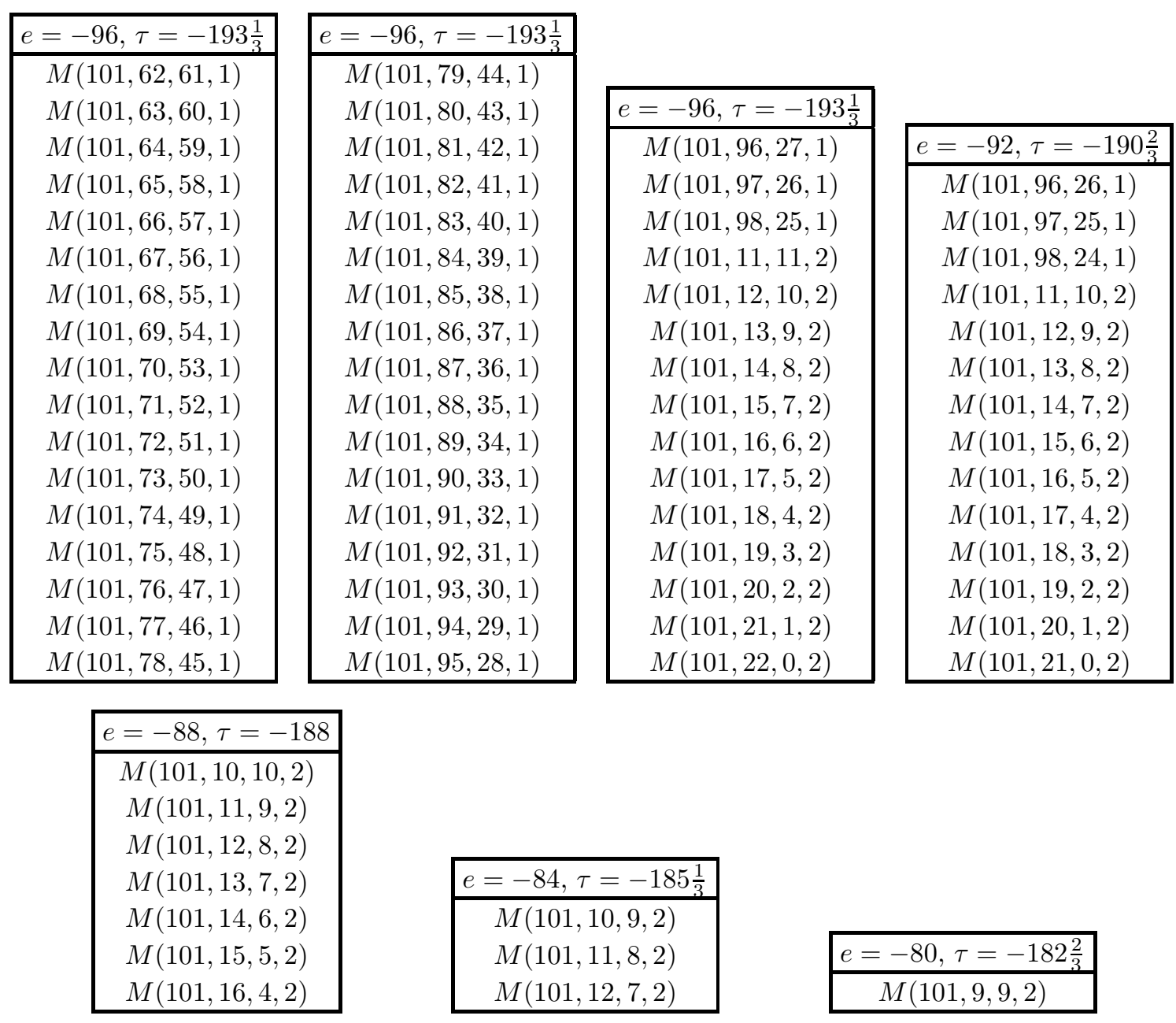

\section{Appendix: technical tools, elementary algebraic and geometric background}

This section contains proofs of all facts that were just stated in the previous sections. The core of the method we use here is a coordinate-free approach in complex hyperbolic geometry. It originates mainly from the works [San] and [HSa]. With this approach, we significantly reduce the amount of calculations involved in the proofs. It happened to be convenient to work in a slightly extended generality, using extensively the positive part of $\mathbb{P}$. This either does not alter or indeed simplifies the proofs. It is worthwhile mentioning that the pseudo-Riemannian geometry of the positive part is important per se, as it is nothing but the geometry of complex geodesics; it is studied more systematically in [AGr1].

Behind several results of this section there is an important and curious geometrical interpretation that we plan to discuss in other articles. It is related to some kind of parallel displacement. (The explicit formulae can be found in [AGr1] and [AGr2].) This concerns the slice identification, the angle between cotranchal bisectors, and the Kähler primitive, for instance.

Sometimes, we prove well-known results. In these cases, the direct references do not simplify the exposition since, usually, we need something developed in the proof.

For the convenience of the reader unfamiliar with complex hyperbolic geometry, we have collected in Subsection 4.1 a series of simple well-known facts. Most of them can be found in [Gol] or [San]. Some of the notation and definitions introduced in this subsection differ from the standard ones. 


\subsection{Preliminaries: hermitian metric, complex geodesics, geodesics, $\mathbb{R}$-planes, and bisectors}

4.1.1. Fix, once and for all, a three-dimensional $\mathbb{C}$-vector space $V$ equipped with a hermitian form $\langle-,-\rangle$ of signature ++- . (In order to deal with the Poincaré disc, one can take $\operatorname{dim}_{\mathbb{C}} V=2$ and signature +-.) Depending on the context, we will use the elements of $V$ to denote the points in the complex projectivization $\mathbb{P}$ of $V$. In general, $\mathbb{P} W$ denotes the complex projectivization of $W \subset V$.

As in 2.1.1, we define

$$
\mathbb{B}:=\{p \in \mathbb{P} \mid\langle p, p\rangle<0\}, \quad \partial_{\infty} \mathbb{B}:=\{p \in \mathbb{P} \mid\langle p, p\rangle=0\}, \quad \overline{\mathbb{B}}:=\mathbb{B} \cup \partial_{\infty} \mathbb{B} .
$$

We recall that every linear map $\varphi \in \operatorname{Lin}_{\mathbb{C}}(\mathbb{C} p, V)$ can be regarded as a tangent vector $t_{\varphi} \in \mathrm{T}_{p} \mathbb{P}$ by defining

$$
t_{\varphi} f:=\left.\frac{d}{d \varepsilon}\right|_{\varepsilon=0} \hat{f}(p+\varepsilon \varphi(p))
$$

for a local smooth function $f$ on $\mathbb{P}$ and its lift $\hat{f}$ to $V$. Indeed, for small $\varepsilon \in \mathbb{R}$, we have a smooth curve $c(\varepsilon) \in \mathbb{P}$ whose lift to $V$ is given by $c_{0}(\varepsilon):=p+\varepsilon \varphi(p)$. The above $t_{\varphi} f$ is nothing but $\dot{c}(0) f$. In this way,

$$
\mathrm{T}_{p} \mathbb{P} \simeq \operatorname{Lin}_{\mathbb{C}}(\mathbb{C} p, V / \mathbb{C} p) .
$$

Let $p$ be nonisotropic, i.e., $p \notin \partial_{\infty} \mathbb{B}$. We have the orthogonal decomposition

$$
V=\mathbb{C} p \oplus p^{\perp}, \quad v=\pi^{\prime}[p] v+\pi[p] v,
$$

where the orthogonal projections

$$
\pi^{\prime}[p] v:=\frac{\langle v, p\rangle}{\langle p, p\rangle} p \in \mathbb{C} p, \quad \pi[p] v:=v-\frac{\langle v, p\rangle}{\langle p, p\rangle} p \in p^{\perp}
$$

do not depend on the choice of a representative $p \in V$. The natural identification

$$
\mathrm{T}_{p} \mathbb{P} \simeq\langle-, p\rangle p^{\perp}
$$

provides a nondegenerate hermitian form on the tangent space $\mathrm{T}_{p} \mathbb{P}$ :

$$
\left\langle v_{p}, w_{p}\right\rangle:=-\langle p, p\rangle\langle v, w\rangle,
$$

where $v, w \in p^{\perp}$ and, for a chosen representative $p \in V$,

$$
v_{p}:=\langle-, p\rangle v \in \mathrm{T}_{p} \mathbb{P}
$$

Obviously, this hermitian form depends smoothly on a nonisotropic $p$.

Lemma 4.1.4. Let $c:[a, b] \rightarrow \mathbb{P}$ be a smooth curve and let $c_{0}:[a, b] \rightarrow V$ be a smooth lift of $c$. If $c\left(t_{0}\right) \notin \partial_{\infty} \mathbb{B}$, then $\dot{c}\left(t_{0}\right)=\left\langle-, c_{0}\left(t_{0}\right)\right\rangle \frac{\pi\left[c\left(t_{0}\right)\right] \dot{c}_{0}\left(t_{0}\right)}{\left\langle c_{0}\left(t_{0}\right), c_{0}\left(t_{0}\right)\right\rangle}$ is the tangent vector to $c$ at $c\left(t_{0}\right)$.

Proof. Denoting $k:=\frac{\left\langle\dot{c}_{0}\left(t_{0}\right), c_{0}\left(t_{0}\right)\right\rangle}{\left\langle c_{0}\left(t_{0}\right), c_{0}\left(t_{0}\right)\right\rangle}$, we have

$$
\dot{c}\left(t_{0}\right) f=\left.\frac{d}{d t}\right|_{t=t_{0}} \hat{f}\left(c_{0}(t)\right)=\left.\frac{d}{d \varepsilon}\right|_{\varepsilon=0} \hat{f}\left(c_{0}\left(t_{0}\right)+\varepsilon \dot{c}_{0}\left(t_{0}\right)\right)=
$$




$$
\begin{gathered}
=\left.\frac{d}{d \varepsilon}\right|_{\varepsilon=0} \hat{f}\left(c_{0}\left(t_{0}\right)+\varepsilon \pi^{\prime}\left[c\left(t_{0}\right)\right] \dot{c}_{0}\left(t_{0}\right)+\varepsilon \pi\left[c\left(t_{0}\right)\right] \dot{c}_{0}\left(t_{0}\right)\right)=\left.\frac{d}{d \varepsilon}\right|_{\varepsilon=0} \hat{f}\left((1+\varepsilon k) c_{0}\left(t_{0}\right)+\varepsilon \pi\left[c\left(t_{0}\right)\right] \dot{c}_{0}\left(t_{0}\right)\right)= \\
=\left.\frac{d}{d \varepsilon}\right|_{\varepsilon=0} \hat{f}\left(c_{0}\left(t_{0}\right)+\frac{\varepsilon}{1+\varepsilon k} \pi\left[c\left(t_{0}\right)\right] \dot{c}_{0}\left(t_{0}\right)\right)=\left.\frac{d}{d \varepsilon}\right|_{\varepsilon=0} \hat{f}\left(c_{0}\left(t_{0}\right)+\varepsilon \pi\left[c\left(t_{0}\right)\right] \dot{c}_{0}\left(t_{0}\right)\right) .
\end{gathered}
$$

4.1.5. Tance. For $p_{1}, p_{2} \in \mathbb{P}$, we define

$$
\operatorname{ta}\left(p_{1}, p_{2}\right):=\frac{\left\langle p_{1}, p_{2}\right\rangle\left\langle p_{2}, p_{1}\right\rangle}{\left\langle p_{1}, p_{1}\right\rangle\left\langle p_{2}, p_{2}\right\rangle}
$$

When one of $p_{1}, p_{2}$ is isotropic, our convention is that $\operatorname{ta}\left(p_{1}, p_{2}\right):=+\infty$ if $\left\langle p_{1}, p_{2}\right\rangle \neq 0$ and $\operatorname{ta}\left(p_{1}, p_{2}\right):=1$ if $\left\langle p_{1}, p_{2}\right\rangle=0$. The concept of tance can be used for expressing distances (see, for example, Corollary 4.1.18 and Lemma 4.1.8).

Let $p_{1}, p_{2} \in \mathbb{B}$. Then $\operatorname{ta}\left(p_{1}, p_{2}\right) \geq 1$ and $\operatorname{ta}\left(p_{1}, p_{2}\right)=1$ if and only if $p_{1}=p_{2}$. Given subsets $X, Y \subset \mathbb{B}$, define the tance between $X$ and $Y$ as

$$
\operatorname{ta}(X, Y):=\inf \{\operatorname{ta}(x, y) \mid x \in X, y \in Y\} .
$$

4.1.6. Polar points to projective lines. For every projective line $\mathrm{L} \subset \mathbb{P}$, there exists a unique point $p \in \mathbb{P}$ such that $\mathrm{L}=\mathbb{P} p^{\perp}$. We call $p$ the polar point to $\mathrm{L}$. By definition, the signature of $\mathrm{L}$ is simply that of the hermitian form on the $\mathbb{C}$-vector subspace in $V$ corresponding to L. The signature of $\mathbb{P} p^{\perp}$ is respectively,,+++-+0 in the cases $p \in \mathbb{B}, p \notin \bar{B}, p \in \partial_{\infty} \mathbb{B}$. Denote by

$$
\mathrm{L}\left\{p_{1}, p_{2}\right\}:=\mathbb{P}\left(\mathbb{C} p_{1}+\mathbb{C} p_{2}\right)
$$

the projective line spanned by two distinct points $p_{1}, p_{2} \in \mathbb{P}$.

Lemma 4.1.7 [Gol, p. 100]. Let $p_{1}, p_{2} \in \mathbb{P}$ be distinct. Then $\mathbb{P} p_{1}^{\perp}$ and $\mathbb{P} p_{2}^{\perp}$ intersect in the polar point $p$ to $\mathrm{L} 2 p_{1}, p_{2} 2$. Moreover,

- $p \in \partial_{\infty} \mathbb{B}$ if and only if $\operatorname{ta}\left(p_{1}, p_{2}\right)=1$;

- $p \in \mathbb{B}$ if and only if $\operatorname{ta}\left(p_{1}, p_{2}\right)<1$ and $p_{1}, p_{2} \notin \overline{\mathbb{B}}$.

Proof. The first assertion is obvious. Sylvester's Criterion [KoM, p. 113] and the definition of $\operatorname{ta}\left(p_{1}, p_{2}\right)$ imply that the signature of $\mathrm{L} 2 p_{1}, p_{2}$ is +0 if and only if $\operatorname{ta}\left(p_{1}, p_{2}\right)=1$. This proves the second assertion. Suppose that $p \in \mathbb{B}$. Then $p_{1}, p_{2} \notin \overline{\mathbb{B}}$ because $p_{1}, p_{2} \in p^{\perp}$. Applying Sylvester's Criterion to the Gram matrix of $p_{1}, p_{2}, p$, we infer that $\operatorname{ta}\left(p_{1}, p_{2}\right)<1$. Conversely, $\operatorname{ta}\left(p_{1}, p_{2}\right)<1$ and $p_{1}, p_{2} \notin \overline{\mathbb{B}}$ imply $p \in \mathbb{B}$ by the same criterion.

The complex geodesics we deal with in Sections 1-3 are by definition of the form $\mathbb{P} p^{\perp} \cap \overline{\mathbb{B}}$ with positive $p$. Two distinct complex geodesics are said to be ultraparallel if they do not intersect. By Lemma 4.1.7, this is equivalent to the inequality $\operatorname{ta}\left(p_{1}, p_{2}\right)>1$ for their polar points $p_{1}$ and $p_{2}$.

The tance between a point and a complex geodesic is given in the following

Lemma 4.1.8 [Gol, p. 197, Theorem 6.1.1]. Let $p \notin \overline{\mathbb{B}}$ and let $q \in \mathbb{B}$. Then $\operatorname{ta}\left(q, \mathbb{P} p^{\perp} \cap \mathbb{B}\right)=$ $1-\operatorname{ta}(p, q)$.

Proof. It follows from $\langle\pi[p] q, \pi[p] q\rangle=\langle\pi[p] q, q\rangle=\langle q, q\rangle(1-\operatorname{ta}(p, q))<0$ that $\pi[p] q \in \mathbb{P} p^{\perp} \cap \mathbb{B}$ and that ta $(\pi[p] q, q)=1-\operatorname{ta}(p, q)$. We choose an orthonormal basis $e_{1}, e_{2}, e_{3}$ in $V$ such that $e_{1}$ and $e_{2}$ represent $p$ and $\pi[p] q$, respectively. We can assume that $q=c e_{1}+e_{2},|c|<1$. Every point in $\mathbb{P} p^{\perp} \cap \mathbb{B}$ can be written in the form $p(z):=e_{2}+z e_{3},|z|<1$. It remains to observe that

$$
\operatorname{ta}(p(z), q)=\frac{1}{\left(-1+|z|^{2}\right)\left(|c|^{2}-1\right)}=\frac{1}{\left(1-|z|^{2}\right)\left(1-|c|^{2}\right)} .
$$


4.1.9. Geodesics and $\mathbb{R}$-planes. Let $S \subset V$ be an $\mathbb{R}$-vector subspace such that the hermitian form is real and nondegenerate on $S$. It is immediate that $\mathbb{C} S \simeq \mathbb{C} \otimes_{\mathbb{R}} S$. Therefore, $\mathbb{P} S=\mathbb{P}_{\mathbb{R}} S$. If $\operatorname{dim}_{\mathbb{R}} S=2$, we call

$$
\mathrm{G} S:=\mathbb{P} S=\mathbb{P}_{\mathbb{R}}^{1} S \simeq \mathbb{S}^{1}
$$

an extended geodesic (or simply a geodesic) [San]. If $\operatorname{dim}_{\mathbb{R}} S=3$, we call

$$
\mathrm{R} S:=\mathbb{P} S=\mathbb{P}_{\mathbb{R}}^{2} S
$$

an extended $\mathbb{R}$-plane (or simply an $\mathbb{R}$-plane) [Gol, p. 80]. When writing G $S$ or $\mathrm{R} S$ (or even $\mathbb{P} S$ ) for a geodesic or real plane, we assume that $S$ is an $\mathbb{R}$-vector subspace such that the hermitian form is real and nondegenerate on $S$. Every geodesic G $S$ spans its projective line L G $S:=\mathbb{P}(\mathbb{C} S)$. By definition, the signature of G $S$ is that of L G $S$. It coincides with the signature of the (hermitian) form on $S$ and can be ++ or +- .

Remark 4.1.10. Let $\mathrm{L}$ be a projective line and let $p \in \mathrm{L}$ be nonisotropic. Then there exists a unique $q \in \mathrm{L}$ orthogonal to $p$, i.e., $\langle p, q\rangle=0$. If $\mathrm{G}$ is a geodesic such that $p \in \mathrm{G} \subset \mathrm{L}$, then $q \in G$.

Sylvester's Criterion implies the following

Remark 4.1.11. Suppose that $\operatorname{ta}\left(g_{1}, g_{2}\right) \neq 0,1$ for some $g_{1}, g_{2} \in \mathbb{P}$. Then there exists a unique extended geodesic $\mathrm{G} \imath g_{1}, g_{2}$ l containing $g_{1}$ and $g_{2}$.

When using the notation $\mathrm{G} 2 g_{1}, g_{2} 2$, we assume by default that $\operatorname{ta}\left(g_{1}, g_{2}\right) \neq 0,1$.

4.1.12. In order to write down the equation of the geodesic $\mathrm{G} 2 g_{1}, g_{2} 2$, we put

$$
b\left(x, g_{1}, g_{2}\right):=\frac{\left\langle g_{1}, x\right\rangle\left\langle x, g_{2}\right\rangle}{\left\langle g_{1}, g_{2}\right\rangle}-\frac{\left\langle g_{2}, x\right\rangle\left\langle x, g_{1}\right\rangle}{\left\langle g_{2}, g_{1}\right\rangle} .
$$

Note that $b\left(x, g_{1}, g_{2}\right)$ does not depend on the choice of representatives $g_{1}, g_{2} \in V$.

Lemma 4.1.13. The geodesic $\mathrm{G}\left\{g_{1}, g_{2}\right\urcorner$ is given in $\mathrm{L}\left\{g_{1}, g_{2}\right\}$ by the equation $b\left(x, g_{1}, g_{2}\right)=0$.

Proof. G $\left\{g_{1}, g_{2} \imath=\mathrm{G} S\right.$ for a suitable real subspace $S \subset V$. The equality $b\left(x, g_{1}, g_{2}\right)=0$ holds for every $x \in S$ since the hermitian form is real on $S$ and we can take $g_{1}, g_{2} \in S$.

Conversely, suppose that $\frac{\left\langle g_{1}, x\right\rangle\left\langle x, g_{2}\right\rangle}{\left\langle g_{1}, g_{2}\right\rangle} \in \mathbb{R}$ for some $x \in \mathrm{L}\left\{g_{1}, g_{2}\right.$. We can assume that $g_{1}, g_{2} \in S$ and that $x=g_{1}+c g_{2}$ for some $c \in \mathbb{C}$. Then $0 \neq\left\langle g_{1}, g_{2}\right\rangle \in \mathbb{R}$ and

$$
0 \equiv\left\langle g_{1}, x\right\rangle\left\langle x, g_{2}\right\rangle \equiv c\left\langle g_{1}, g_{1}\right\rangle\left\langle g_{2}, g_{2}\right\rangle+\bar{c}\left\langle g_{1}, g_{2}\right\rangle\left\langle g_{1}, g_{2}\right\rangle \bmod \mathbb{R} .
$$

If $c \notin \mathbb{R}$, then $\left\langle g_{1}, g_{1}\right\rangle\left\langle g_{2}, g_{2}\right\rangle=\left\langle g_{1}, g_{2}\right\rangle\left\langle g_{1}, g_{2}\right\rangle$. In other words, $\operatorname{ta}\left(g_{1}, g_{2}\right)=1$.

4.1.14. Every geodesic $\mathrm{G}$ of signature +- possesses exactly two isotropic points $v_{1}, v_{2} \in \mathrm{G}$ called the vertices of $\mathrm{G}$. There is a canonical way to describe such a geodesic in terms of its vertices:

Lemma 4.1.15 [Gol, p. 155]. Let $\mathrm{G}$ be a geodesic of signature +-, let $v_{1}, v_{2}$ stand for the vertices of $\mathrm{G}$, and let $g \in \mathrm{G}$ be a point of signature \pm . Then we can choose representatives $v_{1}, v_{2}, g \in V$ such that $g=v_{1}+v_{2}$ and $\left(\begin{array}{cc}0 & \pm \frac{1}{2} \\ \pm \frac{1}{2} & 0\end{array}\right)$ is the Gram matrix of $v_{1}, v_{2}$. The formula $g(t):=t^{-1} v_{1}+t v_{2}, t>0$, describes all points in $\mathrm{G}$ of signature \pm . For all $t$, we have $\langle g(t), g(t)\rangle= \pm 1$.

Proof. We can always choose representatives $v_{1}, v_{2}, g \in V$ providing the required Gram matrix, $g \in \mathbb{R} v_{1}+\mathbb{R} v_{2}$, and $\langle g, g\rangle= \pm 1$. Replacing $g$ by $-g$ if necessary, we obtain $g=t^{-1} v_{1}+t v_{2}$ for a suitable $t>0$. It remains to take $t^{-1} v_{1}, t v_{2}$ for $v_{1}, v_{2}$. 
Lemma 4.1.16. If $p, g_{1}, g_{2} \in \mathbb{B}$, then $\frac{\left\langle g_{1}, p\right\rangle\left\langle p, g_{2}\right\rangle}{\left\langle g_{1}, g_{2}\right\rangle}$ cannot be real nonnegative.

Proof. It suffices to show that $\operatorname{Re} \frac{\left\langle g_{1}, p\right\rangle\left\langle p, g_{2}\right\rangle}{\left\langle g_{1}, g_{2}\right\rangle}<0$ assuming that $g_{1} \neq g_{2} \neq p$. Taking the negative point $\pi[q] p$ in place of $p$, where $q \notin \overline{\mathbb{B}}$ denotes the polar point to $\mathrm{L}\left\{g_{1}, g_{2} \prec\right.$, we can assume that $p \in \mathrm{L}\left\{g_{1}, g_{2}\right.$. Choose representatives such that $\left\langle g_{i}, g_{i}\right\rangle=-1,\left\langle g_{1}, g_{2}\right\rangle=a>1$, and $p=g_{1}+c g_{2}$ for some $c \in \mathbb{C}$. Since $\langle p, p\rangle<0$, we have $2 a \operatorname{Re} c<1+|c|^{2}$. Now,

$$
\operatorname{Re}\left(\left\langle g_{1}, p\right\rangle\left\langle p, g_{2}\right\rangle\right)=\left(a^{2}+1\right) \operatorname{Re} c-a\left(1+|c|^{2}\right)<\frac{\left(1-a^{2}\right)\left(1+|c|^{2}\right)}{2 a}<0
$$

4.1.17. Length of geodesics. Let $\mathrm{G}\left[g_{1}, g_{2}\right] \subset \mathbb{B}$ denote the oriented segment of geodesic joining the points $g_{1}, g_{2} \in \mathbb{B}$. By Lemma 4.1.15, we can parameterize a lift of $\mathrm{G}\left[g_{1}, g_{2}\right]$ to $V$ as $c_{0}(t)=$ $e^{-t} v_{1}+e^{t} v_{2}, t \in[0, a]$, for some $a \geq 0$. It is easy to see that $\dot{c}_{0}(t)=-e^{-t} v_{1}+e^{t} v_{2}$ is orthogonal to $c_{0}(t)$. By Lemma 4.1.4, $\langle\dot{c}(t), \dot{c}(t)\rangle=1$. Therefore, $\ell \mathrm{G}\left[g_{1}, g_{2}\right]=\int_{0}^{a} \sqrt{\langle\dot{c}(t), \dot{c}(t)\rangle} d t=a$. A straightforward calculation shows that $\operatorname{ta}\left(g_{1}, g_{2}\right)=\left(\frac{e^{-a}+e^{a}}{2}\right)^{2}$. We obtain the following result.

Corollary 4.1.18. For $g_{1}, g_{2} \in \mathbb{B}$, we have $\cosh ^{2}\left(\operatorname{dist}\left(g_{1}, g_{2}\right)\right)=\operatorname{ta}\left(g_{1}, g_{2}\right)$.

Now, we can see that the metric on $\mathbb{B}$ coincides, up to the scale factor of 4 , with the one introduced in [Gol, p. 77].

4.1.19. Bisectors. Let $\mathrm{G} S$ be a geodesic and let $f$ stand for the polar point to its projective line L G $S$. The projective cone B over G $S$ with vertex $f$ is said to be an extended bisector (or simply a bisector). The nonisotropic point $f$ is the focus of B. Clearly, $f$ is the only singular point in $\mathrm{B}$. The geodesic $\mathrm{G} S$ is the real spine of $\mathrm{B}$, the projective line $\mathrm{L} \mathrm{G} S$ is the complex spine of $\mathrm{B}$, and the projective line $\mathrm{L} g g, f l$ is the slice of B containing $g \in \mathrm{G} S$. In this way, we obtain the slice decomposition of $\mathrm{B}$ : every point in $\mathrm{B}$ different from the focus belongs to a unique slice of B. By Remark 4.1.10, the polar point to every slice of $B$ belongs to the real spine of $B$. The vertices of the real spine of $\mathrm{B}$ (if exist) are the vertices of $\mathrm{B}$.

We can also describe the bisector $\mathrm{B}$ as $\mathrm{B}=\mathbb{P} W$, where $W:=S+\mathbb{C} f$ is a 4 -dimensional real subspace in $V$. This description immediately provides the meridional decomposition of B : Let us fix some representative $f \in V$. For every $0 \neq c \in \mathbb{C}$, the hermitian form on $S^{\prime}:=S+\mathbb{R} c f$ is real and nondegenerate. The $\mathbb{R}$-plane $\mathrm{R} S^{\prime} \subset \mathrm{B}$ is said

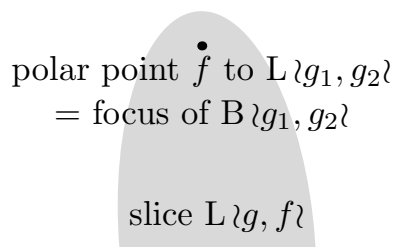

bisector $\mathrm{B}\left\{g_{1}, g_{2}\right\}$

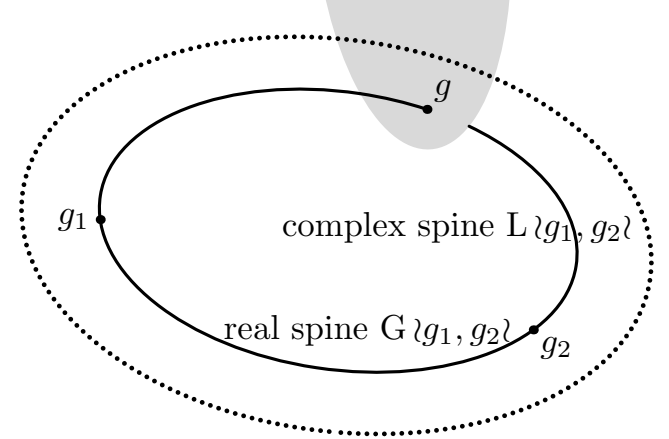
to be a meridian of $\mathrm{B}$. Two different meridians intersect exactly in the focus and in the real spine. Obviously, every point of a bisector belongs to some meridian. Such a meridian is unique if the point is different from the focus and does not lie on the real spine.

It is easy to see that the intersection of a meridian of B with the slice $\mathrm{L}\{g, f<$ of B is a geodesic (unless $g$ is a vertex of the real spine of B) that contains $g$.

It is also possible to define a bisector as the hypersurface equidistant (equitant) from two different points. In this article, we do not need such a definition. 
4.1.20. Remark. Our definition of a bisector differs slightly from the common one. A standardly defined bisector is, in our terms, $\mathrm{B} \cap \overline{\mathrm{B}}$, where $\mathrm{B}$ is a bisector with positive focus. In terms of [Gol, p. 248], the bisectors in our sense are extors, however, not every extor is a bisector in our sense.

We denote by $\mathrm{B}\left\{g_{1}, g_{2}\right.$ ? the bisector with the real spine $\mathrm{G}\left\{g_{1}, g_{2} \imath\right.$. When using the notation $\mathrm{B}\left\{g_{1}, g_{2} 2\right.$, we assume by default that $\operatorname{ta}\left(g_{1}, g_{2}\right) \neq 0,1$.

Lemma 4.1.13 implies the following

Proposition 4.1.21. The bisector $\mathrm{B} l g_{1}, g_{2} l$ is given in $\mathbb{P}$ by the equation $b\left(x, g_{1}, g_{2}\right)=0$.

Lemma 4.1.22 [Gol, p. 165, Theorem 5.2.4]. Let $p_{1}, p_{2} \in \mathbb{P}$ be such that $\operatorname{ta}\left(p_{1}, p_{2}\right) \neq 0,1$ and let $S_{i}:=\mathbb{P} p_{i}^{\perp}, i=1,2$. Then there exists a unique bisector B such that $S_{1}$ and $S_{2}$ are slices of $\mathrm{B}$. The real spine of such $\mathrm{B}$ is $\mathrm{G} \imath p_{1}, p_{2}$.

Proof. The intersection $f$ of the $S_{i}$ 's has to be the focus of the bisector B in question. By Lemma 4.1.7, $\mathrm{L} 2 p_{1}, p_{2}$ is necessarily the complex spine of B. Since the polar point to every

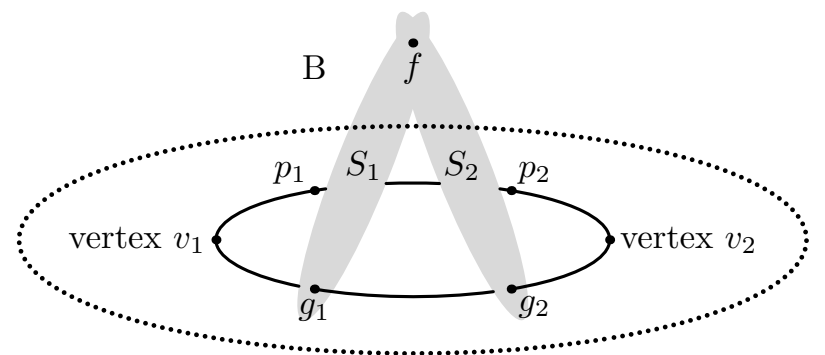
slice of $\mathrm{B}$ belongs to the real spine of $\mathrm{B}$, this real spine contains the points $p_{1}$ and $p_{2}$. By Remark 4.1.11, the real spine of B has to equal $\mathrm{G} \imath p_{1}, p_{2}$. By Remark 4.1.10, the point $g_{i} \in \mathrm{L} \imath p_{1}, p_{2}$ l orthogonal to $p_{i}$ belongs to $\mathrm{G}\left\{p_{1}, p_{2} \imath, i=1,2\right.$. In other words, $S_{i}=\mathrm{L} \imath g_{i}, f \imath$ is a slice of $\mathrm{B}\left\{p_{1}, p_{2}\right.$.

In particular, two ultraparallel complex geodesics $S_{1}$ and $S_{2}$ are slices of a unique bisector B. Obviously, the focus $f$ of such B is positive. The projective cone over $\mathrm{G}\left[g_{1}, g_{2}\right]$ with vertex $f$ is the segment $\mathrm{B}\left[S_{1}, S_{2}\right]$ of $\mathrm{B}$, where $g_{i}$ stands for the intersection of $S_{i}$ with the real spine of B. Using the parameterization given in Lemma 4.1.15 of (positive) polar points to the slices of B, it is easy to show that there exists a complex geodesic $M$ called the middle slice of the segment $\mathrm{B}\left[S_{1}, S_{2}\right]$ such that the reflection in $M$ exchanges $S_{1}$ and $S_{2}$.

Lemma 4.1.23. Suppose that the Gram matrix of $p_{1}, p_{2} \in V$ has the form $\left(\begin{array}{ll}1 & t \\ t & 1\end{array}\right)$ with $t>1$. Then $m:=\frac{p_{1}+p_{2}}{\sqrt{2 t+2}}$ is the polar point to the middle slice $M$ of the segment $\mathrm{B}\left[S_{1}, S_{2}\right]$ and $\langle m, m\rangle=1$, where $S_{i}:=\mathbb{P} p_{i}^{\perp}, i=1,2$.

Proof. It is easy to see that $\langle m, m\rangle=1$. We have (see (2.1.3) for the definition of $R(m)$ )

$$
R(m) p_{1}=2\left\langle p_{1}, m\right\rangle m-p_{1}=\frac{2\left\langle p_{1}, p_{1}+p_{2}\right\rangle}{2 t+2}\left(p_{1}+p_{2}\right)-p_{1}=p_{2} .
$$

For different points $v_{1}, v_{2} \in \partial_{\infty} \mathbb{B}$ and $p \notin \partial_{\infty} \mathbb{B}$, the $\eta$-invariant [Gol, p. 231] is defined as

$$
\eta\left(v_{1}, v_{2}, p\right):=\frac{\left\langle v_{1}, p\right\rangle\left\langle p, v_{2}\right\rangle}{\left\langle v_{1}, v_{2}\right\rangle\langle p, p\rangle} .
$$

The tance between a point and a bisector can be expressed in terms of the $\eta$-invariant:

Lemma 4.1.25 [San, p. 97, Example 2]. Let $\mathrm{B} \imath v_{1}, v_{2}$ l be a bisector with vertices $v_{1}, v_{2} \in \partial_{\infty} \mathbb{B}$ and let $p \in \mathbb{B} \backslash \mathrm{B}\left\{v_{1}, v_{2} 2\right.$. Then ta $\left(p, \mathrm{~B} \prec v_{1}, v_{2} \imath \cap \mathbb{B}\right)=1-\operatorname{Re} \eta\left(v_{1}, v_{2}, p\right)+\left|\eta\left(v_{1}, v_{2}, p\right)\right|$.

Proof. We can assume that $\langle p, p\rangle=-1,\left\langle v_{1}, v_{2}\right\rangle=\frac{1}{2}$, and $r:=\left|z_{1}\right|=\left|z_{2}\right|$, where $z_{i}:=\left\langle p, v_{i}\right\rangle$. By Lemma 4.1.15, the polar points to the slices of signature +- are parameterized by $g(t):=t^{-1} v_{1}+t v_{2}$, $t>0$, with $\langle g(t), g(t)\rangle=1$. By Lemma 4.1.8,

$$
\text { ta }\left(p, \mathbb{P} g(t)^{\perp} \cap \mathbb{B}\right)=1-\operatorname{ta}(p, g(t))=1+2 \operatorname{Re}\left(\bar{z}_{1} z_{2}\right)+t^{-2} r^{2}+t^{2} r^{2} .
$$


This function has a unique minimum at $t=1$. It remains to observe that $\eta\left(v_{1}, v_{2}, p\right)=-2 \bar{z}_{1} z_{2}$.

\subsection{Bisectors}

In this subsection, we explicitly describe the tangent space (at generic points) to subspaces of the form $\mathbb{P} W$, where $W \subset V$ is an $\mathbb{R}$-vector subspace. This tool provides a handy description of the normal vector to a bisector. Then we prove some known facts concerning transversalities. Finally, we observe that a meridional curve (see 2.1.1) depends continuously on its segment of bisector and initial point.

4.2.1. Let $W \subset V$ be an $\mathbb{R}$-vector subspace. We define

$$
d:=\min _{0 \neq w \in W} \operatorname{dim}_{\mathbb{R}} \mathbb{C} w \cap W, \quad D:=\left\{w \in W \mid \operatorname{dim}_{\mathbb{R}} \mathbb{C} w \cap W=d\right\} .
$$

It is easy to show that $D$ is open in $W$, that $\mathbb{P} D$ is smooth and open in $\mathbb{P} W$, and that $\operatorname{dim}_{\mathbb{R}} \mathbb{P} D=$ $\operatorname{dim}_{\mathbb{R}} W-d$.

Lemma 4.2.2. Let $p \in D$ and let $\varphi \in \operatorname{Lin}_{\mathbb{C}}(\mathbb{C} p, V)$. Then $t_{\varphi} \in \mathrm{T}_{p} \mathbb{P} W$ if and only if $\varphi(p) \in \mathbb{C} p+W$.

Proof. Suppose that $\varphi(p) \in W$. For small $\varepsilon \in \mathbb{R}$, we have a smooth curve $c(\varepsilon) \in \mathbb{P} D$ whose lift to $V$ is given by $c_{0}(\varepsilon):=p+\varepsilon \varphi(p) \in W$. Therefore, the tangent vector $t_{\varphi}$ to $c$ belongs to $\mathrm{T}_{p} \mathbb{P} D=\mathrm{T}_{p} \mathbb{P} W$. Such tangent vectors form the whole $\mathrm{T}_{p} \mathbb{P} D$ since

$$
\operatorname{dim}_{\mathbb{R}} \mathbb{P} D=\operatorname{dim}_{\mathbb{R}} W-d=\operatorname{dim}_{\mathbb{R}} W / \mathbb{C} p \cap W .
$$

Corollary 4.2.3. Let $\mathrm{L}$ be a projective line, let $p \in \mathrm{L}$ be nonisotropic, and let $q \in \mathrm{L}$ be orthogonal to $p$. Then $\mathrm{T}_{p} \mathrm{~L}=\langle-, p\rangle \mathbb{C} q$. Let $\mathbb{P} S$ be an $\mathbb{R}$-plane or geodesic and let $p \in S$ be nonisotropic. Then $\mathrm{T}_{p} \mathbb{P} S=\langle-, p\rangle\left(p^{\perp} \cap S\right)$.

As it is easy to see, Lemma 2.1.13 follows from the second part of Corollary 4.2.3.

4.2.4. Orthogonal projective lines. Let $\mathrm{L}_{1}$ be a projective line and let $p \in \mathrm{L}_{1}$ be nonisotropic. Using Corollary 4.2.3, it is easy to show that there exists a unique projective line $\mathrm{L}_{2}$ passing through $p$ and orthogonal to $\mathrm{L}_{1}$ in the sense that $\mathrm{T}_{p} \mathrm{~L}_{1}$ and $\mathrm{T}_{p} \mathrm{~L}_{2}$ are orthogonal. Obviously, $\mathrm{L}_{2}=\mathbb{P} p_{1}^{\perp}$ for some $p_{1} \in \mathrm{L}_{1}$. This means that two distinct projective lines intersecting in a nonisotropic point $p$ are orthogonal at $p$ if and only if their polar points are orthogonal. In this case, $\mathrm{T}_{p} \mathbb{P}=\mathrm{T}_{p} \mathrm{~L}_{1} \oplus \mathrm{T}_{p} \mathrm{~L}_{2}$. In particular, the complex spine of a bisector is orthogonal to every slice of signature different from +0 . This implies that the intersection of such a slice with a meridian is a geodesic orthogonal to the real spine.

4.2.5. Some simple transversalities. In order to show a few simple facts concerning transversalities, we need the following

Lemma 4.2.6. Let $p \in \partial_{\infty} \mathbb{B}$ and let $\varphi \in \operatorname{Lin}_{\mathbb{C}}(\mathbb{C} p, V)$. Then $t_{\varphi} \in \mathrm{T}_{p} \partial_{\infty} \mathbb{B}$ if and only if $\operatorname{Re}\langle\varphi(p), p\rangle=0$.

Proof. We take $q \in V$ such that $\langle p, q\rangle \neq 0$. In some neighbourhood of $p$, the 3 -sphere $\partial_{\infty} \mathbb{B}$ is given by the equation $h(x):=\frac{\langle x, x\rangle}{\langle q, x\rangle\langle x, q\rangle}=0$. The tangent vector $t_{\varphi}$ is simply $\dot{c}(0)$, where $c(\varepsilon)$ is a smooth curve in $\mathbb{P}$ whose lift to $V$ is given by $c_{0}(\varepsilon)=p+\varepsilon \varphi(p)$. It follows from

$$
\dot{c}(0) h=\left.\frac{d}{d \varepsilon}\right|_{\varepsilon=0} h(p+\varepsilon \varphi(p))=\left.\frac{d}{d \varepsilon}\right|_{\varepsilon=0} \frac{\langle p+\varepsilon \varphi(p), p+\varepsilon \varphi(p)\rangle}{\langle q, p+\varepsilon \varphi(p)\rangle\langle p+\varepsilon \varphi(p), q\rangle}=\frac{\langle\varphi(p), p\rangle+\langle p, \varphi(p)\rangle}{\langle p, q\rangle\langle q, p\rangle}
$$

that $\dot{c}(0) h=0$ if and only if $\operatorname{Re}\langle\varphi(p), p\rangle=0$. It remains to observe that such $t_{\varphi}$ 's form a 3 -dimensional $\mathbb{R}$-vector subspace in $\mathrm{T}_{p} \mathbb{P}$. 
Remark 4.2.7 (folklore). Every projective line of signature +- is transversal to $\partial_{\infty} \mathbb{B}$.

Proof. Let $p \notin \overline{\mathbb{B}}$ and let $q \in \mathbb{P} p^{\perp} \cap \partial_{\infty} \mathbb{B}$. Fix some point $q^{\prime} \in \mathbb{P} p^{\perp}$ different from $q$. So, $\mathbb{P} p^{\perp}=\mathrm{L} \prec q, q^{\prime} \prec$ and we can assume that $0 \neq\left\langle q, q^{\prime}\right\rangle \in \mathbb{R}$. Suppose that $t_{\varphi} \in \mathrm{T}_{q} \mathbb{P} p^{\perp} \cap \mathrm{T}_{q} \partial_{\infty} \mathbb{B}$, where $\varphi \in \operatorname{Lin}_{\mathbb{C}}(\mathbb{C} q, V)$. By Lemma $4.2 .2, \varphi(q) \in p^{\perp}=\mathbb{C} q+\mathbb{C} q^{\prime}$. We can change $\varphi$ by adding to $\varphi(q)$ an arbitrary element in $\mathbb{C} q$ since this does not alter $t_{\varphi}$. Hence, we can assume that $\varphi(q) \in \mathbb{C} q^{\prime}$. By Lemma 4.2.6, $\varphi(q) \in \mathbb{R} i q^{\prime}$

Corollary 4.2.8. Every bisector $\mathrm{B}$ is transversal to $\partial_{\infty} \mathbb{B}$.

Proof. Denote by G $S$ the real spine of $\mathrm{B}$ and by $f$, the focus of B. Let $p \in \mathrm{B} \cap \partial_{\infty} \mathbb{B}$. Obviously, $p \neq f$. We can assume $p=s+c f$ for some $0 \neq s \in S$ and $c \in \mathbb{C}$. It follows from $p \in \partial_{\infty} \mathbb{B}$ that $\langle s, s\rangle+|c|^{2}\langle f, f\rangle=0$. Take some $s^{\prime} \in S \backslash \mathbb{R} s$.

Suppose that $t_{\varphi} \in \mathrm{T}_{p} \mathrm{~B} \cap \mathrm{T}_{p} \partial_{\infty} \mathbb{B}$, where $\varphi \in \operatorname{Lin}_{\mathbb{C}}(\mathbb{C} p, V)$. By Lemmas 4.2 .2 and 4.2.6, $\varphi(p) \in$ $\mathbb{C} s+\mathbb{R} s^{\prime}+\mathbb{C} f$ and $\operatorname{Re}\langle\varphi(p), p\rangle=0$. Adding to $\varphi(p)$ an arbitrary element from $\mathbb{C} p$ does not change $t_{\varphi}$. Therefore, we can assume that $\varphi(p) \in \mathbb{R} s^{\prime}+\mathbb{C} f$.

If $c=0$, then $p=s$ is isotropic and $\left\langle s^{\prime}, s\right\rangle \neq 0$. In this case, the equality $\operatorname{Re}\langle\varphi(p), p\rangle=0$ implies $\varphi(p) \in \mathbb{C} f$ and we conclude that $\operatorname{dim}_{\mathbb{R}}\left(\mathrm{T}_{p} \mathrm{~B} \cap \mathrm{T}_{p} \partial_{\infty} \mathbb{B}\right) \leq 2$. If $c \neq 0$, then $s$ is nonisotropic and we can assume that $c=1$ and take $s^{\prime} \in S \backslash \mathbb{R} s$ orthogonal to $s$. In this case, the equality $\operatorname{Re}\langle\varphi(p), p\rangle=0$ implies $\varphi(p) \in \mathbb{R} s^{\prime}+\mathbb{R} i f$.

4.2.9. We now introduce the equation of the tangent space to the bisector $\mathrm{B}\left\{g_{1}, g_{2}\right.$. Define

$$
t\left(v, p, g_{1}, g_{2}\right):=\frac{\left\langle g_{1}, v\right\rangle\left\langle p, g_{2}\right\rangle+\left\langle g_{1}, p\right\rangle\left\langle v, g_{2}\right\rangle}{\left\langle g_{1}, g_{2}\right\rangle}-\frac{\left\langle g_{2}, v\right\rangle\left\langle p, g_{1}\right\rangle+\left\langle g_{2}, p\right\rangle\left\langle v, g_{1}\right\rangle}{\left\langle g_{2}, g_{1}\right\rangle}
$$

Note that $t\left(v, p, g_{1}, g_{2}\right)$ does not depend on the choice of representatives $v, p, g_{1}, g_{2} \in V$ that provides the same linear map $\langle-, p\rangle v$.

Lemma 4.2.10. Let $p \in \mathrm{B} \imath g_{1}, g_{2} \nmid$ be different from the focus $f$ of $\left.\mathrm{B} \imath g_{1}, g_{2}\right)$ and let $\varphi \in \operatorname{Lin}_{\mathbb{C}}(\mathbb{C} p, V)$. Then $\left.t_{\varphi} \in \mathrm{T}_{p} \mathrm{~B} \imath g_{1}, g_{2}\right)$ if and only if $t\left(\varphi(p), p, g_{1}, g_{2}\right)=0$.

Proof. The equality $t\left(\varphi(p), p, g_{1}, g_{2}\right)=0$ remains valid if we choose other representatives $g_{1}, g_{2}, p$ $\in V$. Hence, we can assume that $g_{1}, g_{2} \in S$ and $p \in W$, where $\mathrm{G}\left\{g_{1}, g_{2}\right\}=\mathrm{G} S, \mathrm{~B}\left\{g_{1}, g_{2}\right\}=\mathbb{P} W$, and $W:=S+\mathbb{C} f$. By Lemma 4.2.2, $t_{\varphi} \in \mathrm{T}_{p} \mathrm{~B} \imath g_{1}, g_{2} \imath$ if and only if $\varphi(p) \in \mathbb{C} p+W$.

Suppose that $\varphi(p) \in \mathbb{C} p+W$. Let us show that $t\left(\varphi(p), p, g_{1}, g_{2}\right)=0$. Clearly, $t\left(s, p, g_{1}, g_{2}\right)=0$ for all $s \in S$. Since $\left\langle g_{i}, f\right\rangle=0$ for $i=1,2$, we obtain $t\left(x, p, g_{1}, g_{2}\right)=0$ for all $x \in \mathbb{C} f$. For every $c \in \mathbb{C}$,

$$
t\left(c p, p, g_{1}, g_{2}\right)=\frac{\bar{c}\left\langle g_{1}, p\right\rangle\left\langle p, g_{2}\right\rangle+c\left\langle g_{1}, p\right\rangle\left\langle p, g_{2}\right\rangle}{\left\langle g_{1}, g_{2}\right\rangle}-\frac{\bar{c}\left\langle g_{2}, p\right\rangle\left\langle p, g_{1}\right\rangle+c\left\langle g_{2}, p\right\rangle\left\langle p, g_{1}\right\rangle}{\left\langle g_{2}, g_{1}\right\rangle}=(c+\bar{c}) b\left(p, g_{1}, g_{2}\right)=0
$$

by Proposition 4.1.21. Hence, $t\left(\varphi(p), p, g_{1}, g_{2}\right)=0$.

Conversely, suppose that $t\left(\varphi(p), p, g_{1}, g_{2}\right)=0$. The point $p$ is different from $f$. Interchanging $g_{1}$ and $g_{2}$ if necessary, we can assume that $p=g_{1}+r g_{2}+c^{\prime} f$ for some $r \in \mathbb{R}$ and $c^{\prime} \in \mathbb{C}$. As was shown, $t\left(\mathbb{C} p+\mathbb{C} f, p, g_{1}, g_{2}\right)=0$. It is easy to see that $V=\mathbb{C} g_{2}+\mathbb{C} p+\mathbb{C} f$. Hence, we can assume that $\varphi(p)=c g_{2}$ with $c \in \mathbb{C}$. Since $t\left(c g_{2}, g_{2}, g_{1}, g_{2}\right)=(c+\bar{c}) b\left(g_{2}, g_{1}, g_{2}\right)=0$ and $\left\langle g_{1}, g_{2}\right\rangle \in \mathbb{R}$, we obtain

$$
t\left(c g_{2}, p, g_{1}, g_{2}\right)=t\left(c g_{2}, g_{1}, g_{1}, g_{2}\right)=(\bar{c}-c) \frac{\left\langle g_{1}, g_{2}\right\rangle\left\langle g_{2}, g_{1}\right\rangle-\left\langle g_{1}, g_{1}\right\rangle\left\langle g_{2}, g_{2}\right\rangle}{\left\langle g_{1}, g_{2}\right\rangle}
$$

By Sylvester's Criterion, $t\left(c g_{2}, p, g_{1}, g_{2}\right)=0$ implies $c \in \mathbb{R}$. 
Proposition 4.2.11. Let $p \in \mathrm{B}\left\{g_{1}, g_{2}\right\}$ be nonisotropic and different from the focus $f$ of $\mathrm{B}\left\{g_{1}, g_{2}\right\}$ and let $g \in \mathrm{G} 2 g_{1}, g_{2}$ stand for the polar point to the slice of $\mathrm{B}\left\{g_{1}, g_{2} l\right.$ containing $p$. Then

$$
0 \neq n\left(p, g_{1}, g_{2}\right):=\langle-, p\rangle i\left(\frac{\left\langle p, g_{2}\right\rangle}{\left\langle g_{1}, g_{2}\right\rangle} g_{1}-\frac{\left\langle p, g_{1}\right\rangle}{\left\langle g_{2}, g_{1}\right\rangle} g_{2}\right) \in\langle-, p\rangle \mathbb{C} g
$$

is a normal vector to $\mathrm{B} 2 g_{1}, g_{2}$ at $p$.

Proof. If $n\left(p, g_{1}, g_{2}\right)=0$, then $\left\langle p, g_{i}\right\rangle=0$ for $i=1,2$, implying $p=f$. A contradiction.

Obviously, $n\left(p, g_{1}, g_{2}\right) \in\langle-, p\rangle f^{\perp}$. By Proposition 4.1.21, $b\left(p, g_{1}, g_{2}\right)=0$, implying $n\left(p, g_{1}, g_{2}\right) \in$ $\langle-, p\rangle p^{\perp}$. Clearly, $\mathrm{L}(p, f)$ is the slice of $\mathrm{B}\left\langle g_{1}, g_{2}\right.$ l containing $p$. Therefore, $p^{\perp} \cap f^{\perp}=\mathbb{C} g$.

Take an arbitrary vector $v_{p} \in \mathrm{T}_{p} \mathbb{P}, v \in p^{\perp}$ (see (4.1.3) for the definition). By Lemma 4.2.10, $v_{p} \in \mathrm{T}_{p} \mathrm{~B} \imath g_{1}, g_{2} \imath$ if and only if $t\left(v, p, g_{1}, g_{2}\right)=0$. Since

$$
\left\langle n\left(p, g_{1}, g_{2}\right), v_{p}\right\rangle=-\langle p, p\rangle i\left(\frac{\left\langle g_{1}, v\right\rangle\left\langle p, g_{2}\right\rangle}{\left\langle g_{1}, g_{2}\right\rangle}-\frac{\left\langle g_{2}, v\right\rangle\left\langle p, g_{1}\right\rangle}{\left\langle g_{2}, g_{1}\right\rangle}\right)
$$

and $p$ is nonisotropic, the equality $t\left(v, p, g_{1}, g_{2}\right)=0$ is equivalent to $\operatorname{Re}\left\langle n\left(p, g_{1}, g_{2}\right), v_{p}\right\rangle=0$.

4.2.12. We introduce the orientation of $\mathrm{G}\left\{g_{1}, g_{2}\right\}$ as follows. Let $i=1,2$. If $g_{i} \in \mathbb{B}$, then define $q_{i} \in \mathrm{G}\left\{g_{1}, g_{2}\right\}$ as the point orthogonal to $g_{i}$. Otherwise, put $q_{i}:=g_{i}$. Denote by $a \subset \mathrm{G}\left\{g_{1}, g_{2}\right\}$ the open arc from $q_{2}$ to $q_{1}$ that contains no point orthogonal to $q_{i}, i=1,2$, and such that $a \cap \overline{\mathbb{B}}=\varnothing$. The orientation of $\mathrm{G}\left\{g_{1}, g_{2} 2\right.$ is that of $a$.

Remark 4.2.13. Let $\mathrm{G} \imath g_{1}, g_{2}$ be a geodesic, let $p_{i} \in \mathrm{G} \prec g_{1}, g_{2}$ be orthogonal to $g_{i}$, and let $p$ denote the polar point to $\mathrm{L}\left\{g_{1}, g_{2}\right.$. Suppose that $p_{1}, p_{2} \notin \mathbb{B}$. We have the following cases:

- $p \in \mathbb{B}$. Then $g_{1}, g_{2}, p_{1}, p_{2}$ are in a cyclic order in the circle $\mathrm{G} 2 g_{1}, g_{2}$.

- $p \notin \overline{\mathbb{B}}$. Then $g_{1}, g_{2}, v_{2}, p_{2}, p_{1}, v_{1}$ are in a cyclic order in the circle $\mathrm{G} 2 g_{1}, g_{2} 2$, where $v_{1}, v_{2}$ are the vertices of $\mathrm{G}\left\{g_{1}, g_{2}\right.$ l taken in an appropriate order. (Note that we admit $g_{i}=v_{i}$ or $p_{i}=v_{i}$.)

The bisector $\mathrm{B}\left\{g_{1}, g_{2}\right\}$ is oriented with respect to the orientation of its real spine $\mathrm{G}\left\{g_{1}, g_{2}\right\}$ and the natural (complex) orientation of its slices. Using Remark 4.2.13, it is easy to see that the introduced orientation of a bisector is compatible with the one introduced in 2.1.1.

4.2.14. We will show that the normal vector in Proposition 4.2.11 is in fact normal to the oriented bisector $\mathrm{B}\left\{g_{1}, g_{2}\right.$. It follows from Proposition 4.1.21 that every bisector B $2 g_{1}, g_{2}$ l divides $\mathbb{P}$ into two extended half-spaces given by the inequalities

$$
\operatorname{Im} \frac{\left\langle g_{1}, x\right\rangle\left\langle x, g_{2}\right\rangle}{\left\langle g_{1}, g_{2}\right\rangle} \geq 0, \quad \operatorname{Im} \frac{\left\langle g_{1}, x\right\rangle\left\langle x, g_{2}\right\rangle}{\left\langle g_{1}, g_{2}\right\rangle} \leq 0 .
$$

Lemma 4.2.15. Let $\mathrm{B}\left\{g_{1}, g_{2} \nmid\right.$ be an oriented bisector, let $p_{i} \in \mathrm{G}\left\{g_{1}, g_{2} \nmid\right.$ be the point orthogonal to $g_{i}, i=1,2$, and let $p \in \mathrm{B}\left\{g_{1}, g_{2}\right\}$ be a nonisotropic point different from the focus $f$ of $\mathrm{B}\left\{g_{1}, g_{2} 2\right.$. Denote by $\sigma_{f}$ the signature of $f$. Then

- the inequalities $\operatorname{Im} \frac{\left\langle g_{1}, x\right\rangle\left\langle x, g_{2}\right\rangle}{\left\langle g_{1}, g_{2}\right\rangle} \geq 0$ and $\operatorname{Im} \frac{\left\langle p_{1}, x\right\rangle\left\langle x, p_{2}\right\rangle}{\left\langle p_{1}, p_{2}\right\rangle} \geq 0$ are equivalent,

- the vector $n\left(p, g_{1}, g_{2}\right)$ is normal to the oriented bisector $\mathrm{B} 2 g_{1}, g_{2} 2$,

- the extended half-space given by $\sigma_{f} \operatorname{Im} \frac{\left\langle g_{1}, x\right\rangle\left\langle x, g_{2}\right\rangle}{\left\langle g_{1}, g_{2}\right\rangle} \geq 0$ lies on the side of $n\left(p, g_{1}, g_{2}\right)$.

Proof. By Remark 4.2.13, G $2 g_{1}, g_{2} \curlywedge$ and $\mathrm{G} 2 p_{1}, p_{2} \curlywedge$ coincide as oriented geodesics. By Proposition 4.1.21, we can exclude the case $x \in \mathrm{B}\left\{g_{1}, g_{2}\right\}=\mathrm{B}\left\{p_{1}, p_{2}\right\}$ and reduce the first assertion to the last two. 
Fix $x \notin \mathrm{B}<g_{1}, g_{2}$. We will vary $p, g_{1}, g_{2}$ keeping the following conditions: $p$ belongs to the bisector and satisfies $f \neq p \notin \partial_{\infty} \mathbb{B} ; g_{1}, g_{2}$ belong to the real spine and satisfy ta $\left(g_{1}, g_{2}\right) \neq 0,1$. During the deformation, the orientation of $\mathrm{B}\left\{g_{1}, g_{2}\right.$ l remains the same, the normal vector $n\left(p, g_{1}, g_{2}\right)$ never vanishes, and $\operatorname{Im} \frac{\left\langle g_{1}, x\right\rangle\left\langle x, g_{2}\right\rangle}{\left\langle g_{1}, g_{2}\right\rangle} \neq 0$. Therefore, we can assume that $p \in \mathrm{G}\left\langle g_{1}, g_{2} \imath\right.$, that $g_{1}, g_{2}$ are vertices if $\sigma_{f}=+1$, and that $p=g_{2}$ if $\sigma_{f}=-1$.

The case $\sigma_{f}=+1$. By Lemma 4.1.15, we can choose representatives such that $\left\langle g_{1}, g_{2}\right\rangle= \pm \frac{1}{2}$ and $p=g_{1}+g_{2}$. By Proposition 4.2.11, $n\left(p, g_{1}, g_{2}\right)=\langle-, p\rangle i\left(g_{1}-g_{2}\right)$. By Lemma 4.1.15, a lift of the oriented geodesic $\mathrm{G}\left\{g_{1}, g_{2}\right.$ l is given by $c_{0}(t):=e^{ \pm t} g_{1}+e^{\mp t} g_{2}, t \in \mathbb{R}$. Obviously, $c_{0}(0)=p$. By Lemma 4.1.4, $\dot{c}(0)=\langle-, p\rangle\left(g_{1}-g_{2}\right)$. Since $i \dot{c}(0)=n\left(p, g_{1}, g_{2}\right)$, the vector $n\left(p, g_{1}, g_{2}\right)$ is normal to the oriented bisector $\left.\mathrm{B} \prec g_{1}, g_{2}\right\}$.

The curve $n_{0}(t):=p+t i\langle p, p\rangle\left(g_{1}-g_{2}\right)=(1 \pm t i) g_{1}+(1 \mp t i) g_{2}$ is a lift of a smooth curve $n(t) \in \mathbb{P}$ with $\dot{n}(0)=n\left(p, g_{1}, g_{2}\right)$. It remains to observe that $\operatorname{Im} \frac{\left\langle g_{1}, n(t)\right\rangle\left\langle n(t), g_{2}\right\rangle}{\left\langle g_{1}, g_{2}\right\rangle}=t$.

The case $\sigma_{f}=-1$. We can choose representatives such that $\left\langle g_{i}, g_{i}\right\rangle=1$ and $\left\langle g_{1}, g_{2}\right\rangle=r>0$. By Sylvester's Criterion, $r<1$. By Proposition 4.2.11, $n\left(p, g_{1}, g_{2}\right)=\langle-, p\rangle i\left(\frac{1}{r} g_{1}-g_{2}\right)$. A lift of the oriented geodesic $\mathrm{G}\left\{g_{1}, g_{2}\right\}$ is given by $c_{0}(t):=(1-t) g_{2}+t g_{1}, t \in[0,1]$. (Note that $\left\langle c_{0}(t), g_{2}\right\rangle=0$ would imply $t=\frac{1}{1-r}>1$.) By Lemma 4.1.4, $\dot{c}(0)=\langle-, p\rangle\left(g_{1}-r g_{2}\right)$. The vector $n\left(p, g_{1}, g_{2}\right)$ is normal to the oriented bisector $\mathrm{B} ? g_{1}, g_{2}$ because $i \dot{c}(0)=r n\left(p, g_{1}, g_{2}\right)$.

The curve $n_{0}(t):=g_{2}+t i\left(g_{1}-r g_{2}\right)=t i g_{1}+(1-r t i) g_{2}$ is a lift of a smooth curve $n(t) \in \mathbb{P}$ with $\dot{n}(0)=r n\left(p, g_{1}, g_{2}\right)$. It remains to observe that $\operatorname{Im} \frac{\left\langle g_{1}, n(t)\right\rangle\left\langle n(t), g_{2}\right\rangle}{\left\langle g_{1}, g_{2}\right\rangle}=-t\left(\frac{1-r^{2}}{r}\right)$.

Lemma 4.2.16. An (ideal) meridional curve depends continuously on its segment of bisector and initial point.

Proof. Let $\mathrm{B}\left[g_{1}, g_{2}\right]$ be a segment of bisector with focus $f \notin \overline{\mathbb{B}}$ and $g_{1}, g_{2} \in \mathbb{B}$, let $S_{1}$ denote the initial slice of $\mathrm{B}\left[g_{1}, g_{2}\right]$, i.e., the one containing $g_{1}$, let $p_{1} \in \mathrm{G}\left\{g_{1}, g_{2}\right.$ l be orthogonal to $g_{1}$, and let $m_{0} \in \mathrm{G} z g_{1}, g_{2}$ l denote the polar point to the middle slice of $\mathrm{B}\left[g_{1}, g_{2}\right]$. The meridional curve $b$ of $\mathrm{B}\left[g_{1}, g_{2}\right]$ generated by $p \in S_{1} \cap \overline{\mathbb{B}}$ can be parameterized by $\mathrm{G}\left[g_{1}, g_{2}\right]$ as well as by $\mathrm{G}\left[p_{1}, m_{0}\right]$, where $m \in \mathrm{G}\left[p_{1}, m_{0}\right]$ is the polar point to the middle slice of $\mathrm{B}\left[g_{1}, g\right]$ (in other words, the parameters $g, m$ are related by the equality $\left.g=R(m) g_{1}\right)$. So, by Lemma 2.1.4,

$$
b(g):=-R(m) p .
$$

Since $p \neq f$, we can take $p$ in the form $p=c f+g_{1}$ with $c \in \mathbb{C}$. We assume that $\left\langle g_{1}, g_{1}\right\rangle=-1$. Then $\langle g, g\rangle=-1$ because $g=R(m) g_{1}$. It follows from $\langle m, m\rangle>0$ and (2.1.3) that

$$
\left\langle g, g_{1}\right\rangle=\left\langle R(m) g_{1}, g_{1}\right\rangle=2 \frac{\left\langle g_{1}, m\right\rangle\left\langle m, g_{1}\right\rangle}{\langle m, m\rangle}-\left\langle g_{1}, g_{1}\right\rangle=2 \frac{\left\langle g_{1}, m\right\rangle\left\langle m, g_{1}\right\rangle}{\langle m, m\rangle}+1>0,
$$

implying $\sqrt{\operatorname{ta}\left(g, g_{1}\right)} \frac{\left\langle p, g_{1}\right\rangle}{\left\langle g, g_{1}\right\rangle}=-1$. We have $R(m) f=-f$ since $\langle m, f\rangle=0$. It follows that $b(g)=$ $-R(m) p=c f-g=\pi\left[g_{1}\right] p-g$. Therefore,

$$
b(g)=\pi\left[g_{1}\right] p+\sqrt{\operatorname{ta}\left(g, g_{1}\right)} \frac{\left\langle p, g_{1}\right\rangle}{\left\langle g, g_{1}\right\rangle} g .
$$

Note that this formula does not depend on the choice of representatives $g_{1}, g \in V$ and is linear in $p$. 


\subsection{Bisectors with common slice}

Using the formula for the normal vector to a bisector, we calculate in this subsection the angle between two cotranchal (= having a common slice) bisectors at a point in their common slice (for a geometric interpretation of this angle, see [AGr1]). Then we present a numerical criterion of transversality of such a pair of bisectors. Finally, we prove some properties of transversal cotranchal bisectors including Condition (3) of [AGr3, Theorem 3.5].

Lemma 4.3.1. Let $g \in \mathbb{P} \backslash \partial_{\infty} \mathbb{B}$ and $g_{1}, g_{2} \in \mathbb{P}$ be such that ta $\left(g, g_{i}\right) \neq 0,1$ and let $p \in \mathbb{P} g^{\perp}$ be nonisotropic and different from the foci of $\mathrm{B}\left\{g, g_{i}, i=1,2\right.$. Then

$$
\left\langle n\left(p, g, g_{2}\right), n\left(p, g, g_{1}\right)\right\rangle=-\langle p, p\rangle\langle g, g\rangle \frac{\left\langle g_{1}, p\right\rangle\left\langle p, g_{2}\right\rangle}{\left\langle g_{1}, g\right\rangle\left\langle g, g_{2}\right\rangle} .
$$

Proof. By Proposition 4.2.11, $n\left(p, g, g_{1}\right)=\langle-, p\rangle i \frac{\left\langle p, g_{1}\right\rangle}{\left\langle g, g_{1}\right\rangle} g$ and $n\left(p, g, g_{2}\right)=\langle-, p\rangle i \frac{\left\langle p, g_{2}\right\rangle}{\left\langle g, g_{2}\right\rangle} g$. The result follows from (4.1.2).

The normal vectors $n\left(p, g, g_{1}\right)$ and $n\left(p, g, g_{2}\right)$ are both tangent to the naturally oriented projective line that passes through $p$ and is orthogonal to the common slice $\mathbb{P} g^{\perp}$ of the oriented bisectors $\mathrm{B}\left\{g, g_{i}\right.$, $i=1,2$. Hence, the oriented angle from $n\left(p, g, g_{1}\right)$ to $n\left(p, g, g_{2}\right)$ is simply $\operatorname{Arg}\left\langle n\left(p, g, g_{2}\right), n\left(p, g, g_{1}\right)\right\rangle$. (Recall that the function Arg takes values in $[0,2 \pi)$.)

Corollary 4.3.2. Let $\mathcal{E} \in \mathrm{SU}$ be a regular elliptic isometry and let $e_{1}, e_{2}, e_{3} \in \mathbb{P}$ be the points corresponding to the eigenvectors of $\mathcal{E}$ such that $e_{1} \in \mathbb{B}$. Denote by $E$ the complex geodesic with the polar point $e_{2}$. Let $D$ be a complex geodesic ultraparallel to $E$. Then the oriented angle from $\mathrm{B}[E, D]$ to $\mathrm{B}[E, \mathcal{E} D]$ at $e_{1} \in E$ equals $\operatorname{Arg}\left(\xi_{2} \xi_{1}^{-1}\right)$, where $\xi_{i}$ stands for the eigenvalue of $\mathcal{E}$ corresponding to $e_{i}$, $i=1,2$.

Proof. Denote by $d$ the polar point to $D$. The points $d$ and $e_{1} \in E$ cannot be orthogonal, nor can $d$ and $e_{2}$, since $D$ and $E$ are ultraparallel. Therefore, we can choose representatives $e_{1}, e_{2} \in V$ such that $\left\langle d, e_{1}\right\rangle>0$ and $\left\langle d, e_{2}\right\rangle>0$. By Lemma 4.3.1, the angle in question equals

$$
\operatorname{Arg} \frac{\left\langle d, e_{1}\right\rangle\left\langle e_{1}, \mathcal{E} d\right\rangle}{\left\langle d, e_{2}\right\rangle\left\langle e_{2}, \mathcal{E} d\right\rangle}=\operatorname{Arg} \frac{\left\langle\mathcal{E}^{-1} e_{1}, d\right\rangle}{\left\langle\mathcal{E}^{-1} e_{2}, d\right\rangle}=\operatorname{Arg} \frac{\xi_{1}^{-1}\left\langle e_{1}, d\right\rangle}{\xi_{2}^{-1}\left\langle e_{2}, d\right\rangle}=\operatorname{Arg}\left(\xi_{2} \xi_{1}^{-1}\right)
$$

Criterion 4.3.3. Let $g \notin \overline{\mathbb{B}}$ and $g_{1}, g_{2} \in \mathbb{P}$ be such that $\operatorname{ta}\left(g, g_{i}\right)>1$ for $i=1,2$. The bisectors $\mathrm{B}\left\{g, g_{1}\right\}$ and $\mathrm{B}\left\{g, g_{2}\right.$ lare transversal along $\mathbb{P} g^{\perp} \cap \overline{\mathbb{B}}$ if and only if

$$
\left|\operatorname{Re} \frac{\left\langle g_{1}, g_{2}\right\rangle\langle g, g\rangle}{\left\langle g_{1}, g\right\rangle\left\langle g, g_{2}\right\rangle}-1\right|<\sqrt{1-\frac{1}{\operatorname{ta}\left(g, g_{1}\right)}} \cdot \sqrt{1-\frac{1}{\operatorname{ta}\left(g, g_{2}\right)}} .
$$

Proof. We can choose representatives such that $\langle g, g\rangle=\left\langle g_{1}, g\right\rangle=\left\langle g, g_{2}\right\rangle=1$. Hence, $g_{i}=g+b_{i}$ with $b_{i} \in g^{\perp}$. (Since $b_{i} \in \mathrm{G}\left\{g, g_{i} \cap \cap \mathbb{P} g^{\perp}\right.$, the slice of $\mathrm{B}\left\{g, g_{i} l\right.$ containing $b_{i}$ is $\mathbb{P} g^{\perp}$.) The inequality $\operatorname{ta}\left(g, g_{i}\right)>1$ implies $\left\langle b_{i}, b_{i}\right\rangle<0$. Normalizing the $b_{i}$ 's, we obtain $d_{i}:=\frac{b_{i}}{\sqrt{-\left\langle b_{i}, b_{i}\right\rangle}}$ with $\left\langle d_{i}, d_{i}\right\rangle=-1$. Let $d \in g^{\perp}$ be orthogonal to $d_{2}$, normalized so that $\langle d, d\rangle=1$. Since $d, d_{2}$ form an orthonormal basis in $g^{\perp}$, we have $d_{1}=r d+c d_{2}$ for suitable $r, c \in \mathbb{C}$. Choosing an appropriate representative $d \in V$, we can assume that $r \geq 0$. From $\left\langle d_{1}, d_{1}\right\rangle=-1$, we obtain $r=\sqrt{|c|^{2}-1}$,

$$
d_{1}=\sqrt{|c|^{2}-1} d+c d_{2}, \quad|c| \geq 1 .
$$


Every point $p \in \mathbb{P} g^{\perp} \cap \overline{\mathbb{B}}$ has the form $p=z d+d_{2}$ with $|z| \leq 1$. It follows from $\left\langle p, g_{2}\right\rangle=\left\langle z d+d_{2}, g+b_{2}\right\rangle=$ $\left\langle d_{2}, b_{2}\right\rangle$ that $0 \neq\left\langle p, g_{2}\right\rangle \in \mathbb{R}$. Note that

$$
\frac{\left\langle p, g_{1}\right\rangle}{\sqrt{-\left\langle b_{1}, b_{1}\right\rangle}}=\frac{\left\langle p, b_{1}\right\rangle}{\sqrt{-\left\langle b_{1}, b_{1}\right\rangle}}=\left\langle p, d_{1}\right\rangle=\left\langle z d+d_{2}, \sqrt{|c|^{2}-1} d+c d_{2}\right\rangle=z \sqrt{|c|^{2}-1}-\bar{c}
$$

Let $\varphi \in \operatorname{Lin}_{\mathbb{C}}(\mathbb{C} p, V)$. By Lemma $4.2 .10, t_{\varphi} \in \mathrm{T}_{p} \mathrm{~B} \imath g, g_{i}\left(\right.$ if and only if $t\left(\varphi(p), p, g, g_{i}\right)=0$. In view of $p \in g^{\perp}$, this is equivalent to

$$
\langle g, \varphi(p)\rangle\left\langle p, g_{i}\right\rangle \in \mathbb{R}
$$

By Lemma 4.2.2, $t_{\varphi} \in \mathrm{T}_{p} \mathbb{P} g^{\perp}$ if and only if $\varphi(p) \in g^{\perp}$. Therefore, considering $t_{\varphi}$ modulo $\mathrm{T}_{p} \mathbb{P} g^{\perp}$, we can assume that $\varphi(p) \in \mathbb{C} g$.

Suppose that $\varphi(p)=a g \neq 0, a \in \mathbb{C}$. Since $0 \neq\left\langle p, g_{2}\right\rangle \in \mathbb{R}$, the condition $\langle g, \varphi(p)\rangle\left\langle p, g_{2}\right\rangle \in \mathbb{R}$ is equivalent to $a \in \mathbb{R}$. Consequently, the condition $t_{\varphi} \in \mathrm{T}_{p} \mathrm{~B}\left\{g, g_{1} \imath \cap \mathrm{T}_{p} \mathrm{~B}\left\{g, g_{2} \zeta\right.\right.$ is equivalent to the requirements that $a \in \mathbb{R}$ and $\left\langle p, g_{1}\right\rangle \in \mathbb{R}$. In terms of $z$, the latter takes the form $z \sqrt{|c|^{2}-1}-\bar{c} \in \mathbb{R}$. The existence of $z$ with $|z| \leq 1$ meeting the last condition means that $\sqrt{|c|^{2}-1} \geq|\operatorname{Im} c|$. This is equivalent to $|\operatorname{Re} c| \geq 1$. We can see that the bisectors $\mathrm{B}\left\{g, g_{1}\right.$ l and $\mathrm{B}\left\{g, g_{2}\right.$ lare transversal along $\mathbb{P} g^{\perp} \cap \overline{\mathbb{B}}$ if and only if $|\operatorname{Re} c|<1$.

It remains to observe that $\sqrt{1-\frac{1}{\operatorname{ta}\left(g, g_{i}\right)}}=\sqrt{1-\left\langle g_{i}, g_{i}\right\rangle}=\sqrt{-\left\langle b_{i}, b_{i}\right\rangle}$ and that

$$
-c \sqrt{-\left\langle b_{1}, b_{1}\right\rangle} \sqrt{-\left\langle b_{2}, b_{2}\right\rangle}=\left\langle d_{1}, d_{2}\right\rangle \sqrt{-\left\langle b_{1}, b_{1}\right\rangle} \sqrt{-\left\langle b_{2}, b_{2}\right\rangle}=\left\langle b_{1}, b_{2}\right\rangle=\left\langle g_{1}, g_{2}\right\rangle-1=\frac{\left\langle g_{1}, g_{2}\right\rangle\langle g, g\rangle}{\left\langle g_{1}, g\right\rangle\left\langle g, g_{2}\right\rangle}-1
$$

In order to prove some fundamental properties of transversal cotranchal bisectors, we need the following technical

Remark 4.3.4. Let $v_{1}, v_{1}^{\prime}, v_{2}, v_{2}^{\prime} \in \partial_{\infty} \mathbb{B}$ be such that $v_{i} \neq v_{i}^{\prime}$ for all $i=1,2$ and let $g \in\left(\mathrm{G} \imath v_{1}, v_{1}^{\prime} \prec \cap\right.$ $\mathrm{G}\left\langle v_{2}, v_{2}^{\prime} \imath\right) \backslash \overline{\mathbb{B}}$. Then there exist representatives $v_{1}, v_{1}^{\prime}, v_{2}, v_{2}^{\prime}, g \in V$ such that $\left\langle v_{i}, v_{i}^{\prime}\right\rangle=\frac{1}{2}$ and $g=v_{i}+v_{i}^{\prime}$ for all $i$. Such representatives satisfy the condition $\operatorname{Re}\left\langle v_{1}, v_{2}\right\rangle \leq \frac{1}{4}$ or $\operatorname{Re}\left\langle v_{1}^{\prime}, v_{2}\right\rangle \leq \frac{1}{4}$. Moreover, the inequality $\operatorname{Re}\left\langle v_{1}, v_{2}\right\rangle \leq 2\left|\left\langle v_{1}, v_{2}\right\rangle\right|^{2}$ holds, the equality being valid exactly when $v_{1}, v_{1}^{\prime}, v_{2}, v_{2}^{\prime}, g$ belong to a same projective line.

Proof. By Lemma 4.1.15, we take representatives $g_{1}, g_{2} \in V$ of $g$ and representatives $v_{i}, v_{i}^{\prime} \in V$ such that $\left\langle v_{i}, v_{i}^{\prime}\right\rangle=\frac{1}{2}$ and $g_{i}=v_{i}+v_{i}^{\prime}$ for $i=1,2$. Since $\left|g_{1}\right|=\left|g_{2}\right|$, we have $g_{1}=c g_{2}$ with $|c|=1$. It remains to take $c v_{2}, c v_{2}^{\prime}$ in place of $v_{2}, v_{2}^{\prime}$.

It follows from $\left\langle g, v_{2}\right\rangle=\frac{1}{2}$ that $\left\langle v_{1}, v_{2}\right\rangle+\left\langle v_{1}^{\prime}, v_{2}\right\rangle=\frac{1}{2}$, implying that $\operatorname{Re}\left\langle v_{1}, v_{2}\right\rangle \leq \frac{1}{4}$ or $\operatorname{Re}\left\langle v_{1}^{\prime}, v_{2}\right\rangle \leq \frac{1}{4}$.

The rest follows from Sylvester's Criterion: the determinant of the Gram matrix of $g, v_{1}, v_{2}$ equals $\frac{1}{2} \operatorname{Re}\left\langle v_{1}, v_{2}\right\rangle-\left|\left\langle v_{1}, v_{2}\right\rangle\right|^{2}$.

Lemma 4.3.5 (compare with [Hsi, p. 97, Theorem 3.3]). Let $\mathrm{B}_{1}$ and $\mathrm{B}_{2}$ be two bisectors with positive foci and let $S$ be a common slice of $\mathrm{B}_{1}$ and $\mathrm{B}_{2}$ of signature +- . Suppose that $\mathrm{B}_{1}$ and $\mathrm{B}_{2}$ are transversal along $S \cap \overline{\mathbb{B}}$. Then $\mathrm{B}_{1} \cap \overline{\mathbb{B}}, \mathrm{B}_{2} \cap \overline{\mathbb{B}}$, and $\partial_{\infty} \mathbb{B}$ are transversal and $\mathrm{B}_{1} \cap \mathrm{B}_{2} \cap \overline{\mathbb{B}}=S \cap \overline{\mathbb{B}}$.

Proof. Let $g \notin \overline{\mathbb{B}}$ denote the polar point to $S$ and let $v_{i}, v_{i}^{\prime} \in \partial_{\infty} \mathbb{B}$ denote the vertices of $\mathrm{B}_{i}, i=1,2$. By Remark 4.3.4, we can assume that $\operatorname{Re} u \leq 2|u|^{2}, \operatorname{Re} u \leq \frac{1}{4},\left\langle v_{i}, v_{i}^{\prime}\right\rangle=\frac{1}{2}$, and $g=v_{i}+v_{i}^{\prime}$ for $i=1,2$, where $u:=\left\langle v_{1}, v_{2}\right\rangle$. Since $\mathrm{B}_{i}=\mathrm{B} \imath g, v_{i}$, we have $\operatorname{Re} u>0$ by Criterion 4.3.3.

Every point in $\mathrm{G} ? g, v_{i}$ but $v_{i}$ has the form

$$
g_{i}\left(t_{i}\right):=g+\left(t_{i}-1\right) v_{i}
$$


with $t_{i} \in \mathbb{R}$. It is easy to see that $\left\langle g_{i}\left(t_{i}\right), g_{i}\left(t_{i}\right)\right\rangle=t_{i}$. Therefore, $g_{i}\left(t_{i}\right), t_{i}>0$, runs over all polar points to the slices of $\mathrm{B}_{i}$ of signature +- .

Let us show that the slices of signature +- of the bisectors $B_{1}$ and $B_{2}$ that are different from their common slice $S$ do not intersect in $\overline{\mathbb{B}}$. By Lemma 4.1.7, it suffices to prove that the inequality $\operatorname{ta}\left(g_{1}\left(t_{1}\right), g_{2}\left(t_{2}\right)\right)>1$ is valid for $t_{1}, t_{2}>0$ unless $t_{1}=t_{2}=1$. Since

$$
2\left\langle g_{1}\left(t_{1}\right), g_{2}\left(t_{2}\right)\right\rangle=t_{1}+t_{2}+2\left(t_{1}-1\right)\left(t_{2}-1\right) u
$$

and $\left\langle g_{i}\left(t_{i}\right), g_{i}\left(t_{i}\right)\right\rangle=t_{i}$, we obtain

$$
4 t_{1} t_{2} \operatorname{ta}\left(g_{1}\left(t_{1}\right), g_{2}\left(t_{2}\right)\right)=\left(t_{1}+t_{2}\right)^{2}+4\left(t_{1}+t_{2}\right)\left(t_{1}-1\right)\left(t_{2}-1\right) \operatorname{Re} u+4\left(t_{1}-1\right)^{2}\left(t_{2}-1\right)^{2}|u|^{2} .
$$

Hence, the inequality ta $\left(g_{1}\left(t_{1}\right), g_{2}\left(t_{2}\right)\right)>1$ is equivalent to the inequality

$$
\left(t_{1}-t_{2}\right)^{2}+4\left(t_{1}+t_{2}\right)\left(t_{1}-1\right)\left(t_{2}-1\right) \operatorname{Re} u+4\left(t_{1}-1\right)^{2}\left(t_{2}-1\right)^{2}|u|^{2}>0
$$

which can be rewritten in the form

$$
\left(t_{1}-t_{2}\right)^{2}+2\left(t_{1}^{2}-1\right)\left(t_{2}^{2}-1\right) \operatorname{Re} u+2\left(t_{1}-1\right)^{2}\left(t_{2}-1\right)^{2}\left(2|u|^{2}-\operatorname{Re} u\right)>0 .
$$

Since $\operatorname{Re} u>0$ and $2|u|^{2} \geq \operatorname{Re} u$, this inequality is valid if $t_{1}, t_{2}>1$ or if $0<t_{1}, t_{2}<1$. Therefore, we can assume that $0<t_{1}<1<t_{2}$. In this case, taking into account the inequalities $\operatorname{Re} u \leq \frac{1}{4}$ and $2|u|^{2} \geq \operatorname{Re} u$, it suffices to observe that $\left(t_{1}-t_{2}\right)^{2}>\frac{1}{2}\left(1-t_{1}^{2}\right)\left(t_{2}^{2}-1\right)$. We have shown that the slices of signature +- of $\mathrm{B}_{1}$ and $\mathrm{B}_{2}$ do not intersect.

The bisectors $\mathrm{B}_{1}$ and $\mathrm{B}_{2}$ cannot have a common vertex $v$. Otherwise, they would have the same real spine $\mathrm{G} r g, v 2$ and would coincide.

Note that $u$ cannot be real. This would contradict the inequalities $0<\operatorname{Re} u \leq \frac{1}{4}$ and $\operatorname{Re} u \leq 2|u|^{2}$. It is easy to see that no vertex of one bisector belongs to the other bisector. Indeed, $v_{2} \in \mathrm{B}_{1}$ means that $\left\langle g_{1}\left(t_{1}\right), v_{2}\right\rangle=0$ for a suitable $t_{1}>0$. This can be written as $\frac{1}{2}+\left(t_{1}-1\right) u=0$, implying that $u$ is real. Similarly, $v_{2}^{\prime} \in \mathrm{B}_{1}$ implies that $\frac{t_{1}}{2}+\left(1-t_{1}\right) u=0$ for a suitable $t_{1}>0$.

Thus, $\mathrm{B}_{1} \cap \mathrm{B}_{2} \cap \overline{\mathbb{B}}=S \cap \overline{\mathbb{B}}$.

The transversality follows from Corollary 4.2.8 and Remark 4.2.7.

Lemma 4.3.6. Let $\mathrm{B}_{1}$ and $\mathrm{B}_{2}$ be bisectors with positive foci. Suppose that they possess a common slice $S$ of signature +- and that they are transversal along $S \cap \overline{\mathbb{B}}$. Then, for any $\vartheta>0$, there exists some $\varepsilon>0$ such that, for every $p \in \mathrm{B}_{2} \cap \mathbb{B}$, the inequality $\operatorname{ta}\left(p, \mathrm{~B}_{1} \cap \mathbb{B}\right)<1+\varepsilon^{2}$ implies the inequality $\operatorname{ta}(p, S \cap \mathbb{B})<1+\vartheta^{2}$.

Proof. Let $g \notin \overline{\mathbb{B}}$ denote the polar point to $S$ and let $v_{i}, v_{i}^{\prime} \in \partial_{\infty} \mathbb{B}$ denote the vertices of $\mathrm{B}_{i}, i=1,2$. By Remark 4.3.4, we can assume that $\operatorname{Re} u \leq 2|u|^{2}$, Re $u \leq \frac{1}{4},\left\langle v_{i}, v_{i}^{\prime}\right\rangle=\frac{1}{2}$, and $g=v_{i}+v_{i}^{\prime}$ for $i=1,2$, where $u:=\left\langle v_{1}, v_{2}\right\rangle$. In particular, we have $\left\langle v_{i}, g\right\rangle=\left\langle v_{i}^{\prime}, g\right\rangle=\frac{1}{2}$. (The equality $\operatorname{Re} u=2|u|^{2}$ means that $\mathrm{B}_{1}$ and $\mathrm{B}_{2}$ have the same complex spine. In this case, the fact is easier and will be proven later.) Since $\mathrm{B}_{i}=\mathrm{B}\left\{g, v_{i}\right.$, we have $\operatorname{Re} u>0$ by Criterion 4.3.3.

We assume that $\operatorname{Re} u<2|u|^{2}$. Therefore, $g, v_{1}, v_{2}$ are $\mathbb{C}$-linearly independent. The point

$$
f:=v_{1}+(4 u-1) v_{2}-2 u g
$$

is the focus of $\mathrm{B}_{2}$ because $\left\langle f, v_{2}\right\rangle=\langle f, g\rangle=0$. Define

$$
k:=\sqrt{4|u|^{2}-2 \operatorname{Re} u} .
$$


It is easy to see that

$$
\langle f, f\rangle=\left\langle v_{1}, f\right\rangle=(4 \bar{u}-1) u-\bar{u}=k^{2}, \quad\left\langle f, v_{1}^{\prime}\right\rangle=\left\langle f, g-v_{1}\right\rangle=-k^{2} .
$$

Let us parameterize the points in $\mathrm{B}_{2} \cap \mathbb{B}$. By Lemma 4.1.15, the negative points in the real spine $\mathrm{G}\left\langle v_{2}, v_{2}^{\prime}\right\}$ of $\mathrm{B}_{2}$ are parameterized by $g(t):=t^{-1} v_{2}-t v_{2}^{\prime}, t>0$. Since $\left\langle\frac{f}{k}, \frac{f}{k}\right\rangle=1$, every point in $\mathrm{B}_{2} \cap \mathbb{B}$ takes the form $p(t, z):=\frac{z}{k} f+g(t)$, where $t>0$ and $|z|<1$. (The point $p(t, z)$ belongs to the slice of $\mathrm{B}_{2}$ that contains $g(t)$.) Calculating straightforwardly, we obtain

$$
\begin{gathered}
\langle p(t, z), g\rangle=\langle g(t), g\rangle=\frac{t^{-1}-t}{2}, \quad\left\langle v_{1}, g(t)\right\rangle=\left\langle v_{1}, t^{-1} v_{2}-t\left(g-v_{2}\right)\right\rangle=\left(t^{-1}+t\right) u-\frac{t}{2}, \\
\left\langle g(t), v_{1}^{\prime}\right\rangle=\left\langle g(t), g-v_{1}\right\rangle=\frac{t^{-1}}{2}-\left(t^{-1}+t\right) \bar{u}, \quad\langle g(t), g(t)\rangle=-1, \quad\langle p(t, z), p(t, z)\rangle=|z|^{2}-1, \\
\left\langle v_{1}, p(t, z)\right\rangle=k \bar{z}+\left(t^{-1}+t\right) u-\frac{t}{2}, \quad\left\langle p(t, z), v_{1}^{\prime}\right\rangle=-k z+\frac{t^{-1}}{2}-\left(t^{-1}+t\right) \bar{u} .
\end{gathered}
$$

By Lemma 4.1.8,

$$
\operatorname{ta}(p(t, z), S \cap \mathbb{B})=1-\operatorname{ta}(p(t, z), g)=1+\frac{\left(t^{-1}-t\right)^{2}}{4\left(1-|z|^{2}\right)}=1+h^{2}, \quad h:=\frac{\left|t^{-1}-t\right|}{2 \sqrt{1-|z|^{2}}} .
$$

In order to express explicitly the tance ta $\left(p(t, z), \mathrm{B}_{1} \cap \mathbb{B}\right)$, we need to calculate the $\eta$-invariant $(4.1 .24)$ :

$$
\eta\left(v_{1}, v_{1}^{\prime}, p(t, z)\right)=\frac{\left(2\left(t^{-1}+t\right) u+2 k \bar{z}-t\right)\left(2\left(t^{-1}+t\right) \bar{u}+2 k z-t^{-1}\right)}{2\left(1-|z|^{2}\right)}=a+i b,
$$

where

$$
\begin{gathered}
a:=\frac{1+\left(t^{-1}+t\right)^{2} k^{2}+4 k^{2}|z|^{2}+2\left(t^{-1}+t\right) k\left(\left(4 u_{0}-1\right) z_{0}-4 u_{1} z_{1}\right)}{2\left(1-|z|^{2}\right)} \\
b:=\left(t^{-1}-t\right) \cdot \frac{\left(t^{-1}+t\right) u_{1}-k z_{1}}{1-|z|^{2}}, \quad u=u_{0}-i u_{1}, \quad z=z_{0}-i z_{1}, \quad u_{0}, u_{1}, z_{0}, z_{1} \in \mathbb{R} .
\end{gathered}
$$

We have to estimate the tance ta $\left(p(t, z), \mathrm{B}_{1} \cap \mathbb{B}\right)$ in terms of $h$. To this end, we introduce

$$
c:=\left|4 u_{0}-1\right|+4\left|u_{1}\right|, \quad d:=\left(4 u_{0}-1\right) z_{0}-4 u_{1} z_{1}, \quad e:=\sqrt{1-|z|^{2}}
$$

rewrite $a$ and $b$ (using the equality $t^{-1}+t=2 \sqrt{e^{2} h^{2}+1}$ ) as

$$
a=\frac{1+8 k^{2}+4 k^{2} e^{2} h^{2}-4 k^{2} e^{2}+4 k d \sqrt{e^{2} h^{2}+1}}{2 e^{2}}, \quad b= \pm h \frac{4 u_{1} \sqrt{e^{2} h^{2}+1}-2 k z_{1}}{e},
$$

and observe that the inequalities

$$
0<c, \quad|d| \leq c, \quad 0<e \leq 1, \quad 0 \leq h, \quad 0<k<2\left|u_{1}\right|
$$

are valid. Only the last inequality requires a proof: It follows from $0<u_{0} \leq \frac{1}{4}<\frac{1}{2}$ that $\left(\frac{1}{2}-2 u_{0}\right)^{2}<\frac{1}{4}$. Hence, $k=\sqrt{4 u_{1}^{2}+4 u_{0}^{2}-2 u_{0}}=\sqrt{4 u_{1}^{2}+\left(\frac{1}{2}-2 u_{0}\right)^{2}-\frac{1}{4}}<2\left|u_{1}\right|$. 
Suppose that ta $\left(p(t, z), \mathrm{B}_{1} \cap \mathbb{B}\right)<1+\varepsilon^{2}$ for some $\varepsilon>0$. By Lemma 4.1.25, ta $\left(p(t, z), \mathrm{B}_{1} \cap \mathbb{B}\right)=$ $1-a+|a+i b|$ in view of (4.3.7). Therefore, $b^{2}<\varepsilon^{4}+2 a \varepsilon^{2}$, which can be written in the form

$$
\left(4 h u_{1} \sqrt{e^{2} h^{2}+1}-2 h k z_{1}\right)^{2}-\left(2 \varepsilon k \sqrt{e^{2} h^{2}+1}+\varepsilon d\right)^{2}<\varepsilon^{2}\left(1+4 k^{2}+\varepsilon^{2} e^{2}-4 k^{2} e^{2}-d^{2}\right)
$$

taking the expressions (4.3.8) into account. Using an inequality of the type $(|A|-|B|)^{2}-(|C|+|D|)^{2} \leq$ $(A-B)^{2}-(C+D)^{2}$ and the fact that $|d| \leq c$ and $e^{2} \leq 1$, we conclude that

$$
\left(4 h\left|u_{1}\right| \sqrt{e^{2} h^{2}+1}-2 h k\left|z_{1}\right|\right)^{2}-\left(2 \varepsilon k \sqrt{e^{2} h^{2}+1}+\varepsilon c\right)^{2}<\varepsilon^{2}\left(1+4 k^{2}+\varepsilon^{2}\right) .
$$

In view of $\left|z_{1}\right|<1 \leq \sqrt{e^{2} h^{2}+1}$ and $k<2\left|u_{1}\right|$, this implies that

$$
\left(4 h\left|u_{1}\right| \sqrt{e^{2} h^{2}+1}-2 h k\right)^{2}-\left(2 \varepsilon k \sqrt{e^{2} h^{2}+1}+\varepsilon c\right)^{2}<\varepsilon^{2}\left(1+4 k^{2}+\varepsilon^{2}\right) .
$$

This inequality can be converted into

$$
\left(\left(4 h\left|u_{1}\right|-2 \varepsilon k\right) \sqrt{e^{2} h^{2}+1}-(2 h k+\varepsilon c)\right)\left(\left(4 h\left|u_{1}\right|+2 \varepsilon k\right) \sqrt{e^{2} h^{2}+1}-(2 h k-\varepsilon c)\right)<\varepsilon^{2}\left(1+4 k^{2}+\varepsilon^{2}\right) .
$$

Given $\vartheta>0$, we have to find some $\varepsilon>0$ such that the inequality ta $\left(p, \mathrm{~B}_{1} \cap \mathbb{B}\right)<1+\varepsilon^{2}$ implies $h<\vartheta$. First, we require that $\varepsilon \leq \vartheta$. We assume now that $4 h\left|u_{1}\right|-2 \varepsilon k \geq 0$ because, otherwise, the inequality $h<\varepsilon$ follows from $0<k<2\left|u_{1}\right|$ and implies that $h<\vartheta$. Next, we require that $\varepsilon \leq \frac{4\left|u_{1}\right|-2 k}{c+2 k} \vartheta$. Now, we assume that $4 h\left|u_{1}\right|-2 \varepsilon k \geq 2 h k+\varepsilon c$ (otherwise, the inequality $h<\vartheta$ follows) and conclude that

$$
\left(4 h\left|u_{1}\right|-2 \varepsilon k\right) \sqrt{e^{2} h^{2}+1}-(2 h k+\varepsilon c) \geq 0
$$

in view of $\sqrt{e^{2} h^{2}+1} \geq 1$. Requiring that $\varepsilon \leq 1$, we can deduce from (4.3.9) that

$$
\left(\left(4 h\left|u_{1}\right|-2 \varepsilon k\right) \sqrt{e^{2} h^{2}+1}-(2 h k+\varepsilon c)\right)^{2}<\varepsilon^{2}\left(2+4 k^{2}\right)
$$

which, in its turn, implies $4 h\left|u_{1}\right|-2 \varepsilon k-2 h k-\varepsilon c<\varepsilon \sqrt{2+4 k^{2}}$ due to $1 \leq \sqrt{e^{2} h^{2}+1}$ and $4 h\left|u_{1}\right|-2 \varepsilon k \geq$ $2 h k+\varepsilon c$. We obtain $h\left(4\left|u_{1}\right|-2 k\right)<\varepsilon\left(\sqrt{2+4 k^{2}}+2 k+c\right)$ and, therefore, $h<\frac{\sqrt{2+4 k^{2}}+2 k+c}{4\left|u_{1}\right|-2 k} \varepsilon$. Finally, we require that $\varepsilon \leq \frac{4\left|u_{1}\right|-2 k}{\sqrt{2+4 k^{2}}+2 k+c} \vartheta$.

The complete list of requirements concerning $\varepsilon$ is the following:

$$
\varepsilon \leq \vartheta, \quad \varepsilon \leq \frac{4\left|u_{1}\right|-2 k}{c+2 k} \vartheta, \quad \varepsilon \leq 1, \quad \varepsilon \leq \frac{4\left|u_{1}\right|-2 k}{\sqrt{2+4 k^{2}}+2 k+c} \vartheta
$$

(Note that none of these inequalities involves $t$ or $z$.)

Now, we consider the case when $\mathrm{B}_{1}$ and $\mathrm{B}_{2}$ have the same complex spine. Let $f$ be the common focus of $\mathrm{B}_{1}$ and $\mathrm{B}_{2}$. We assume $\langle f, f\rangle=1$. Every point in $\mathrm{B}_{2} \cap \mathbb{B}$ has the form $p(t, z):=z f+t^{-1} v_{2}-t v_{2}^{\prime}$, $t>0,|z|<1$. We have

$$
\langle p(t, z), g\rangle=\frac{t^{-1}-t}{2}, \quad\left\langle v_{1}, p(t, z)\right\rangle=\left\langle v_{1}, t^{-1} v_{2}-t\left(g-v_{2}\right)\right\rangle=\left(t^{-1}+t\right) u-\frac{t}{2},
$$




$$
\left\langle p(t, z), v_{1}^{\prime}\right\rangle=\left\langle p(t, z), g-v_{1}\right\rangle=\frac{t^{-1}}{2}-\left(t^{-1}+t\right) \bar{u}, \quad\langle p(t, z), p(t, z)\rangle=|z|^{2}-1 .
$$

By Lemma 4.1.8,

$$
\operatorname{ta}(p(t, z), S \cap \mathbb{B})=1-\operatorname{ta}(p(t, z), g)=1+\frac{\left(t^{-1}-t\right)^{2}}{4\left(1-|z|^{2}\right)}=1+h^{2}, \quad h:=\frac{\left|t^{-1}-t\right|}{2 \sqrt{1-|z|^{2}}}
$$

Taking into account that $\operatorname{Re} u=2|u|^{2}$, we calculate the $\eta$-invariant (4.1.24) :

$$
\eta\left(v_{1}, v_{1}^{\prime}, p(t, z)\right)=\frac{\left(2\left(t^{-1}+t\right) u-t\right)\left(2\left(t^{-1}+t\right) \bar{u}-t^{-1}\right)}{2\left(1-|z|^{2}\right)}=\frac{1+2 i\left(t^{2}-t^{-2}\right) u_{1}}{2\left(1-|z|^{2}\right)}=a+i b
$$

where

$$
a:=\frac{1}{2\left(1-|z|^{2}\right)}, \quad b:=\frac{\left(t^{2}-t^{-2}\right) u_{1}}{1-|z|^{2}}, \quad u_{1}:=\operatorname{Im} u .
$$

Suppose that ta $\left(p(t, z), \mathrm{B}_{1} \cap \mathbb{B}\right)<1+\varepsilon^{2}$ for some $\varepsilon>0$. By Lemma 4.1.25, ta $\left(p(t, z), \mathrm{B}_{1} \cap \mathbb{B}\right)=$ $1-a+|a+i b|$ in view of (4.3.10). Therefore, $b^{2}<\varepsilon^{4}+2 a \varepsilon^{2}$, which can be written in the form

$$
\frac{\left(t^{-2}-t^{2}\right)^{2} u_{1}^{2}}{1-|z|^{2}}<\varepsilon^{2}\left(\varepsilon^{2}\left(1-|z|^{2}\right)+1\right) .
$$

Since $|z|<1$ and $t+t^{-1} \geq 2$, we obtain $16 h^{2} u_{1}^{2}<\varepsilon^{2}\left(\varepsilon^{2}+1\right)$. Note that $u$ cannot be real: this would contradict $\operatorname{Re} u=2|u|^{2}$ and $0<\operatorname{Re} u \leq \frac{1}{4}$. Requiring that $\varepsilon^{2} \leq 15$ and $\varepsilon \leq\left|u_{1}\right| \vartheta$, we arrive at $h<\vartheta$.

\subsection{Triangles of bisectors}

This subsection begins with a numerical criterion of transversality of an oriented triangle of bisectors. Then we establish the path-connectedness of the space of oriented transversal triangles. Finally, we calculate the trace of the holonomy of an oriented triangle (see 2.5.1) and prove that counterclockwiseoriented transversal triangles can be neither R-parabolic nor trivial.

4.4.1. Throughout this subsection, $g_{1}, g_{2}, g_{3}$ denote positive points such that $\operatorname{ta}\left(g_{i}, g_{j}\right)>1$ for $i \neq j$. Put

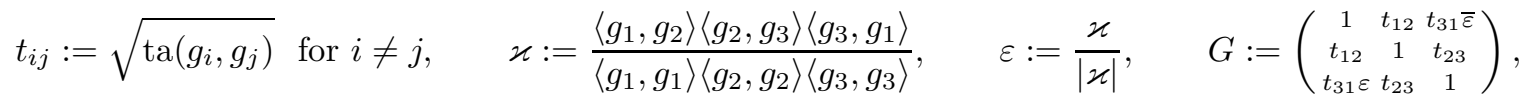

$$
\begin{aligned}
& \varepsilon_{0}:=\operatorname{Re} \varepsilon, \quad \varepsilon_{1}:=\operatorname{Im} \varepsilon, \quad d:=\operatorname{det} G=1+2 t_{12} t_{23} t_{31} \varepsilon_{0}-t_{12}^{2}-t_{23}^{2}-t_{31}^{2}, \quad S S_{i}:=\mathbb{P} g_{i}^{\perp} .
\end{aligned}
$$

We fix suitable representatives such that $G$ is the Gram matrix of $g_{1}, g_{2}, g_{3} \in V$. The numbers $t_{i j}$ 's and $\varepsilon$ are invariant under the action of PU on the ordered triples $\left(g_{1}, g_{2}, g_{3}\right)$ and constitute a complete set of invariants. Indeed, if the triples $\left(g_{1}, g_{2}, g_{3}\right)$ and $\left(g_{1}^{\prime}, g_{2}^{\prime}, g_{3}^{\prime}\right)$ have the same Gram matrix, then there exists some $X \in \mathrm{U}$ such that $X g_{i}=g_{i}^{\prime}$. By Sylvester's Criterion [KoM, p. 113], all the values of the $t_{i j}$ 's and $\varepsilon$ subject to the conditions $t_{i j}>1,|\varepsilon|=1$, and $d \leq 0$ are possible. The equality $d=0$ means that $g_{1}, g_{2}, g_{3}$ lie on the same projective line.

Recall that the triangle of bisectors $\Delta\left(S_{1}, S_{2}, S_{3}\right)$ is transversal if the bisectors B $\left\{g_{i}, g_{j}\right\}$ and B $\left\{g_{i}, g_{k}\right\}$ are transversal along their common slice $S_{i} \cap \overline{\mathbb{B}}$ for all $i, j, k$. The triangle $\Delta\left(S_{1}, S_{2}, S_{3}\right)$ is said to be counterclockwise-oriented if the oriented angle from $\mathrm{B}\left[S_{2}, S_{3}\right]$ to $\mathrm{B}\left[S_{2}, S_{1}\right]$ at some point $p \in S_{2} \cap \mathbb{B}$ does not exceed $\pi$ (see Definition 2.3.5). 
Criterion 4.4.2. A transversal triangle of bisectors is counterclockwise-oriented if and only if $\varepsilon_{1}<0$. Suppose that $1<t_{12} \leq t_{23}, t_{31}$. Then $\Delta\left(S_{1}, S_{2}, S_{3}\right)$ is transversal if and only if $t_{12}^{2} \varepsilon_{0}^{2}+t_{23}^{2}+t_{31}^{2}<$ $1+2 t_{12} t_{23} t_{31} \varepsilon_{0}$.

Proof. For the first assertion, we measure the oriented angle from $\mathrm{B}\left[S_{2}, S_{3}\right]$ to $\mathrm{B}\left[S_{2}, S_{1}\right]$ at the point $p:=\pi\left[g_{2}\right] g_{1}=g_{1}-t_{12} g_{2} \in S_{2}$. (It follows from $t_{12}>1$ that $p \in S_{2} \cap \mathbb{B}$.) By Lemma 4.3.1, this angle equals

$$
\operatorname{Arg} \frac{\left\langle g_{3}, p\right\rangle\left\langle p, g_{1}\right\rangle}{\left\langle g_{3}, g_{2}\right\rangle\left\langle g_{2}, g_{1}\right\rangle}=\operatorname{Arg}\left(\left(t_{31} \varepsilon-t_{12} t_{23}\right)\left(1-t_{12}^{2}\right)\right)=\operatorname{Arg}\left(t_{12} t_{23}-t_{31} \varepsilon\right)
$$

where Arg takes values in $[0,2 \pi)$. The triangle is counterclockwise-oriented exactly when $\operatorname{Im}\left(t_{12} t_{23}-\right.$ $\left.t_{31} \varepsilon\right)>0$, that is, when $\varepsilon_{1}<0$.

By Criterion 4.3.3, the transversality of $\mathrm{B}\left\{g_{1}, g_{2} \nmid\right.$ and $\mathrm{B}\left\{g_{2}, g_{3} \nmid\right.$ along $S_{2} \cap \overline{\mathbb{B}}$ is equivalent to the inequality

$$
\left|\operatorname{Re} \frac{t_{31} \bar{\varepsilon}}{t_{12} t_{23}}-1\right|<\sqrt{1-\frac{1}{t_{12}^{2}}} \cdot \sqrt{1-\frac{1}{t_{23}^{2}}} .
$$

This inequality is equivalent to $\left(t_{31} \varepsilon_{0}-t_{12} t_{23}\right)^{2}<\sqrt{\left(t_{12}^{2}-1\right)\left(t_{23}^{2}-1\right)}$, that is, to

$$
t_{12}^{2}+t_{23}^{2}+t_{31}^{2} \varepsilon_{0}^{2}<1+2 t_{12} t_{23} t_{31} \varepsilon_{0} .
$$

By symmetry, the other two transversalities are equivalent to the inequalities

$$
t_{12}^{2}+t_{23}^{2} \varepsilon_{0}^{2}+t_{31}^{2}<1+2 t_{12} t_{23} t_{31} \varepsilon_{0}, \quad t_{12}^{2} \varepsilon_{0}^{2}+t_{23}^{2}+t_{31}^{2}<1+2 t_{12} t_{23} t_{31} \varepsilon_{0} .
$$

The last inequality implies the other two because $t_{12} \leq t_{23}, t_{31}$.

The path-connectedness of the space of oriented transversal triangles of bisectors is the subject of the following

Lemma 4.4.3. The region given in $\mathbb{R}^{4}\left(e, t_{1}, t_{2}, t_{3}\right)$ by the inequalities $1<t_{1} \leq t_{2}, t_{3}$ and $t_{1}^{2} e^{2}+t_{2}^{2}+$ $t_{3}^{2}<1+2 t_{1} t_{2} t_{3} e \leq t_{1}^{2}+t_{2}^{2}+t_{3}^{2}$ is path-connected.

Proof. The inequalities in the lemma imply $e^{2}<1$ and, hence, $t_{1} e<t_{2} t_{3}$. The inequality $t_{1}^{2} e^{2}+t_{2}^{2}+$ $t_{3}^{2}<1+2 t_{1} t_{2} t_{3} e$ can be rewritten as $\left(t_{2} t_{3}-t_{1} e\right)^{2}<\left(t_{2}^{2}-1\right)\left(t_{3}^{2}-1\right)$. Therefore, it is equivalent to the inequalities $t_{2} t_{3}-\sqrt{\left(t_{2}^{2}-1\right)\left(t_{3}^{2}-1\right)}<t_{1} e \leq t_{2} t_{3}$. Now, from the inequality $1 \leq t_{2} t_{3}-\sqrt{\left(t_{2}^{2}-1\right)\left(t_{3}^{2}-1\right)}$ implied by $1<t_{2} t_{3}$, we conclude that $1<t_{1} e$. In particular, $0<e<1$.

The inequalities

$$
1<t_{1} \leq t_{2}, t_{3}, \quad t_{2} t_{3}-\sqrt{\left(t_{2}^{2}-1\right)\left(t_{3}^{2}-1\right)}<t_{1} e \leq t_{2} t_{3}, \quad 1+2 t_{1} t_{2} t_{3} e \leq t_{1}^{2}+t_{2}^{2}+t_{3}^{2}
$$

are equivalent to those in the lemma. We have seen that they imply $0<e<1<t_{1} e$.

Suppose that $e, t_{1}, t_{2}, t_{3}$ satisfy the inequalities in the lemma. Keeping the inequalities (4.4.4), we will vary the $t_{i}$ 's until we reach $t_{1}=t_{2}=t_{3}$ or $1+2 t_{1} t_{2} t_{3} e=t_{1}^{2}+t_{2}^{2}+t_{3}^{2}$. Without loss of generality, we can assume that $t_{2} \leq t_{3}$. It follows from the inequality $t_{2}<t_{2} t_{3} e$ (implied by $1<t_{1} e$ and $t_{1} \leq t_{3}$ ) that the function $f(x):=x^{2}+t_{2}^{2}+t_{3}^{2}-2 x t_{2} t_{3} e-1$ is decreasing in $x \in\left[t_{1}, t_{2}\right]$. Increasing $t_{1}$ and keeping the inequalities (4.4.4) and $t_{2} \leq t_{3}$, we can reach a point such that either $t_{1}=t_{2}$ or $f\left(t_{1}\right)=0$. The latter means that $1+2 t_{1} t_{2} t_{3} e=t_{1}^{2}+t_{2}^{2}+t_{3}^{2}$.

In the case of $t_{1}=t_{2}$, we can rewrite the condition $t_{1}=t_{2}$ and the inequalities in the lemma as

$$
1<t_{1}=t_{2} \leq t_{3}, \quad 2 t_{1}^{2}\left(t_{3} e-1\right) \leq t_{3}^{2}-1<t_{1}^{2}\left(2 t_{3} e-e^{2}-1\right) .
$$


As we saw, these conditions imply that $0<e<1<t_{1} e$. Hence, $0<t_{3} e-1$ and $0<2 t_{3} e-e^{2}-1$. Now, we increase $t_{1}=t_{2}$ keeping conditions (4.4.5) and reach a point where $t_{1}=t_{2}=t_{3}$ or $2 t_{1}^{2}\left(t_{3} e-1\right)=t_{3}^{2}-1$. The latter means that $1+2 t_{1} t_{2} t_{3} e=t_{1}^{2}+t_{2}^{2}+t_{3}^{2}$.

In the case of $t_{1}=t_{2}=t_{3}$, the inequalities (4.4.4) take the form

$$
1<t_{1}=t_{2}=t_{3}, \quad 1<t_{1} e \leq t_{1}^{2}, \quad t_{1} e \leq \frac{3 t_{1}^{2}-1}{2 t_{1}^{2}}
$$

It follows from $\frac{3 t_{1}^{2}-1}{2 t_{1}^{2}}<t_{1}^{2}$ (implied by $\left.1<t_{1}\right)$ that we can increase $e$ and keep conditions (4.4.6) until $t_{1} e=\frac{3 t_{1}^{2}-1}{2 t_{1}^{2}}$. Again, we reach a point satisfying the equality $1+2 t_{1} t_{2} t_{3} e=t_{1}^{2}+t_{2}^{2}+t_{3}^{2}$.

It remains to show that the region given by the inequalities in the lemma and by the equality $1+2 t_{1} t_{2} t_{3} e=t_{1}^{2}+t_{2}^{2}+t_{3}^{2}$ is path-connected. Excluding $e$ with the help of the equality, we can describe this region as the one given in $\mathbb{R}^{3}\left(t_{1}, t_{2}, t_{3}\right)$ by the inequalities

$$
1<t_{1} \leq t_{2}, t_{3}, \quad t_{1}^{2}+t_{2}^{2}+t_{3}^{2}<1+2 t_{1} t_{2} t_{3}
$$

Assuming that $t_{2} \leq t_{3}$, we obtain $t_{2}<t_{1} t_{3}$. Therefore, keeping the inequalities (4.4.7), we can increase $t_{2}$ until $t_{2}=t_{3}$. Now, the inequalities (4.4.7) are equivalent to the conditions $1<t_{1} \leq t_{2}=t_{3}$.

4.4.8. Trace of holonomy. Let us recall the definition of the holonomy $\varphi$ of $\Delta\left(S_{1}, S_{2}, S_{3}\right)$. Put

$$
m_{1}:=\frac{g_{1}+g_{2}}{\sqrt{2 t_{12}+2}}, \quad m_{2}:=\frac{g_{2}+g_{3}}{\sqrt{2 t_{23}+2}}, \quad m_{3}:=\frac{\varepsilon g_{1}+g_{3}}{\sqrt{2 t_{31}+2}}
$$

By Lemma 4.1.23, $R_{i}:=R\left(m_{i}\right)$ is the reflection (see (2.1.3) for the definition) in the middle slice of the segment $\mathrm{B}\left[S_{i}, S_{i+1}\right]$ and $\left\langle m_{i}, m_{i}\right\rangle=1$ (the indices are modulo 3 ). Define $\varphi:=R_{3} R_{2} R_{1} \in \mathrm{SU}$. It is easy to see that $R_{1} g_{1}=g_{2}, R_{2} g_{2}=g_{3}$, and $R_{3} g_{3}=\varepsilon g_{1}$. Hence,

$$
\varphi g_{1}=\varepsilon g_{1}
$$

In particular, $g_{1}^{\perp}$ is stable under $\varphi$. Therefore, the isometry $\varphi$ induces an isometry $\psi \in \operatorname{SU}\left(g_{1}^{\perp}\right)$ of the complex geodesic $\mathbb{P} g_{1}^{\perp}$.

Lemma 4.4.11. $|\operatorname{tr} \psi|=\sqrt{2\left(1+\varepsilon_{0}\right)}\left(1-\frac{d}{\left(t_{12}+1\right)\left(t_{23}+1\right)\left(t_{31}+1\right)}\right)$.

Proof. Since $\varphi \in \mathrm{SU}$ and $\varphi g_{1}=\varepsilon g_{1}$, it suffices to show that

$$
\operatorname{tr} \varphi=\varepsilon-(1+\bar{\varepsilon})\left(1-\frac{d}{\left(t_{12}+1\right)\left(t_{23}+1\right)\left(t_{31}+1\right)}\right)
$$

Indeed, (4.4.12) implies that $\operatorname{tr} \psi= \pm(\sqrt{\varepsilon}+\overline{\sqrt{\varepsilon}})\left(1-\frac{d}{\left(t_{12}+1\right)\left(t_{23}+1\right)\left(t_{31}+1\right)}\right)$ because $\operatorname{det} \varphi=1$ and $\varphi g_{1}=\varepsilon g_{1}$.

Define the linear maps $\varphi_{i} \in \operatorname{Lin}_{\mathbb{C}}(V, V)$ by the rule $\varphi_{i} x:=2\left\langle x, m_{i}\right\rangle m_{i}$ and denote $g_{i j}:=\left\langle m_{i}, m_{j}\right\rangle$, $i, j=1,2,3$. Obviously, $R_{i}=\varphi_{i}-1$ and

$$
\varphi_{j} \varphi_{i} x=4\left\langle x, m_{i}\right\rangle g_{i j} m_{j}, \quad \varphi_{k} \varphi_{j} \varphi_{i} x=8\left\langle x, m_{i}\right\rangle g_{i j} g_{j k} m_{k}
$$


Considering the orthogonal decompositions $V=\mathbb{C} m_{i} \oplus m_{i}^{\perp}$, we obtain

$$
\operatorname{tr} \varphi_{i}=2, \quad \operatorname{tr}\left(\varphi_{j} \varphi_{i}\right)=4 g_{i j} g_{j i}, \quad \operatorname{tr}\left(\varphi_{k} \varphi_{j} \varphi_{i}\right)=8 g_{i j} g_{j k} g_{k i} .
$$

It follows from $\varphi=R_{3} R_{2} R_{1}=\varphi_{3} \varphi_{2} \varphi_{1}-\varphi_{3} \varphi_{2}-\varphi_{3} \varphi_{1}-\varphi_{2} \varphi_{1}+\varphi_{3}+\varphi_{2}+\varphi_{1}-1$ that

$$
\operatorname{tr} \varphi=8 g_{12} g_{23} g_{31}-4 g_{23} g_{32}-4 g_{13} g_{31}-4 g_{12} g_{21}+3
$$

(compare with [Pra]). From (4.4.9), we obtain

$$
\begin{gathered}
g_{12}=\frac{1+t_{12}+t_{23}+t_{31} \bar{\varepsilon}}{2 \sqrt{\left(t_{12}+1\right)\left(t_{23}+1\right)}}, \quad g_{23}=\frac{1+t_{23}+t_{31}+t_{12} \bar{\varepsilon}}{2 \sqrt{\left(t_{23}+1\right)\left(t_{31}+1\right)}}, \quad g_{31}=\frac{\varepsilon+t_{31} \varepsilon+t_{12} \varepsilon+t_{23}}{2 \sqrt{\left(t_{31}+1\right)\left(t_{12}+1\right)}}, \\
4 g_{23} g_{32}=4 \operatorname{ta}\left(m_{2}, m_{3}\right)=\frac{\left(1+t_{23}+t_{31}\right)^{2}+t_{12}^{2}+2 t_{12}\left(1+t_{23}+t_{31}\right) \varepsilon_{0}}{\left(t_{23}+1\right)\left(t_{31}+1\right)}= \\
=2+\frac{2 t_{12}\left(1+t_{23}+t_{31}+t_{23} t_{31}\right) \varepsilon_{0}-d}{\left(t_{23}+1\right)\left(t_{31}+1\right)}=2+2 t_{12} \varepsilon_{0}-\frac{d\left(t_{12}+1\right)}{\left(t_{12}+1\right)\left(t_{23}+1\right)\left(t_{31}+1\right)} .
\end{gathered}
$$

By symmetry,

$$
4 g_{13} g_{31}=2+2 t_{23} \varepsilon_{0}-\frac{d\left(t_{23}+1\right)}{\left(t_{12}+1\right)\left(t_{23}+1\right)\left(t_{31}+1\right)}, \quad 4 g_{12} g_{21}=2+2 t_{31} \varepsilon_{0}-\frac{d\left(t_{31}+1\right)}{\left(t_{12}+1\right)\left(t_{23}+1\right)\left(t_{31}+1\right)} .
$$

It is possible to show with a straightforward calculation that

$$
\begin{gathered}
8 g_{12} g_{23} g_{31}\left(t_{12}+1\right)\left(t_{23}+1\right)\left(t_{31}+1\right)= \\
=\left(t_{12}+1\right)\left(t_{23}+1\right)\left(t_{31}+1\right)\left(2\left(t_{12}+t_{23}+t_{31}\right) \varepsilon_{0}+2+\varepsilon-\bar{\varepsilon}\right)-d\left(t_{12}+t_{23}+t_{31}+2-\bar{\varepsilon}\right) .
\end{gathered}
$$

In other words,

$$
8 g_{12} g_{23} g_{31}=2\left(t_{12}+t_{23}+t_{31}\right) \varepsilon_{0}+2+\varepsilon-\bar{\varepsilon}-\frac{d\left(t_{12}+t_{23}+t_{31}+2-\bar{\varepsilon}\right)}{\left(t_{12}+1\right)\left(t_{23}+1\right)\left(t_{31}+1\right)} .
$$

Now, from (4.4.13), we infer (4.4.12).

4.4.14. Parabolic holonomy. Recall that a triangle $\Delta\left(S_{1}, S_{2}, S_{3}\right)$ is parabolic or trivial if the isometry $\psi$ (see 4.4.8) is parabolic or the identity. An oriented parabolic triangle is L-parabolic if $\psi$ moves the points in $S_{1} \cap \partial_{\infty} \mathbb{B}$ (different from the fixed one) in the clockwise direction.

Lemma 4.4.15. Let $\Delta\left(S_{1}, S_{2}, S_{3}\right)$ be a counterclockwise-oriented transversal triangle. Suppose that $\Delta\left(S_{1}, S_{2}, S_{3}\right)$ is parabolic or trivial. Then $\Delta\left(S_{1}, S_{2}, S_{3}\right)$ is L-parabolic.

Proof. Denote $p:=\pi\left[g_{1}\right] g_{2} \in S_{1}$. It follows from $t_{12}>1$ that $p \in S_{1} \cap \mathbb{B}$. Let $q \in S_{1} \cap \partial_{\infty} \mathbb{B}$ denote a fixed point of $\varphi$. We assume that $\langle p, q\rangle=1$ and define $p^{\prime}:=\langle p, p\rangle q-p \in p^{\perp}$. Since $\left\langle p^{\prime}, p^{\prime}\right\rangle=-\langle p, p\rangle$, every point in $S_{1} \cap \overline{\mathbb{B}}$ has the form

$$
p(z):=z p^{\prime}+p, \quad z \in \mathbb{C}, \quad|z| \leq 1,
$$

in the orthogonal basis $p, p^{\prime} \in g_{1}^{\perp}$. Clearly, $p(1) \sim q$, where $\sim$ stands for $\mathbb{C}^{*}$-proportionality.

Since $\varphi$ is either parabolic or trivial on $S_{1}=\mathrm{L} \imath p, q 2$ with fixed point $q$, we have

$$
\varphi p=u p+u i v q, \quad \varphi q=u q
$$


for suitable $u, v \in \mathbb{C}, u \neq 0$. This implies that

$$
\operatorname{tr} \varphi=2 u+\varepsilon
$$

because $\varphi g_{1}=\varepsilon g_{1}$ by (4.4.10). From $\varphi \in \mathrm{SU},\langle p, q\rangle=1$, and $\langle q, q\rangle=0$, we conclude that

$$
1=\langle p, q\rangle=\langle\varphi p, \varphi q\rangle=|u|^{2}, \quad\langle p, p\rangle=\langle\varphi p, \varphi p\rangle=|u|^{2}(\langle p, p\rangle-i \bar{v}+i v)=\langle p, p\rangle-2 \operatorname{Im} v
$$

Hence, $v \in \mathbb{R}$.

Let us understand how does $\varphi$ act on $S_{1}$ in terms of $z$ :

$$
\begin{aligned}
& \varphi(p(z))=z \varphi(\langle p, p\rangle q-p)+\varphi p \sim z\langle p, p\rangle q+(1-z)(p+i v q)= \\
& =\frac{z\langle p, p\rangle+(1-z) i v}{\langle p, p\rangle}(\langle p, p\rangle q-p)+\frac{\langle p, p\rangle+(1-z) i v}{\langle p, p\rangle} p \sim p\left(z^{\prime}\right),
\end{aligned}
$$

where

$$
z^{\prime}:=\frac{z\langle p, p\rangle+(1-z) i v}{\langle p, p\rangle+(1-z) i v}
$$

In particular, $\varphi(p(-1)) \sim p\left(z_{0}\right)$ with $z_{0}:=\frac{2 i v-\langle p, p\rangle}{2 i v+\langle p, p\rangle}$ and $\operatorname{Im} z_{0}=\frac{4 v\langle p, p\rangle}{|2 i v+\langle p, p\rangle|^{2}}$. We see that $\varphi$ moves $p(-1)$ in the clockwise direction exactly when $\operatorname{Im} z_{0}>0$, i.e., if and only if $v<0$. In other words, the triangle is L-parabolic if and only if $v<0$. It is easy to see from (4.4.16) that the triangle is trivial if and only if $v=0$.

Thus, we have to show that $v<0$.

It follows from $\langle p, q\rangle=1$ and (4.4.16) that $\langle\varphi p, p\rangle=u(\langle p, p\rangle+i v)$, i.e., $v=\operatorname{Im}\left(u^{-1}\langle\varphi p, p\rangle\right)$. By (4.4.12),

$$
\operatorname{tr} \varphi=2 u+\varepsilon=\varepsilon-(1+\bar{\varepsilon})\left(1-\frac{d}{\left(t_{12}+1\right)\left(t_{23}+1\right)\left(t_{31}+1\right)}\right)
$$

with $d \leq 0$. Consequently, $v$ and $\operatorname{Im}(-(1+\varepsilon)\langle\varphi p, p\rangle)$ have the same signs.

Let us calculate $\langle\varphi p, p\rangle$ in terms of the Gram matrix $G$. We only need to find $\varphi g_{2}$ since $p=g_{2}-t_{12} g_{1}$ and $\varphi g_{1}=\varepsilon g_{1}$ by (4.4.10). Using (4.4.9) and (2.1.3), we see that

$$
R_{1} x=\left\langle x, g_{1}+g_{2}\right\rangle \frac{g_{1}+g_{2}}{t_{12}+1}-x, \quad R_{2} x=\left\langle x, g_{2}+g_{3}\right\rangle \frac{g_{2}+g_{3}}{t_{23}+1}-x, \quad R_{3} x=\left\langle x, \varepsilon g_{1}+g_{3}\right\rangle \frac{\varepsilon g_{1}+g_{3}}{t_{31}+1}-x
$$

for all $x \in V$. So, $R_{1} g_{2}=g_{1}, R_{2} g_{1}=\frac{t_{12}+t_{31} \bar{\varepsilon}}{t_{23}+1}\left(g_{2}+g_{3}\right)-g_{1}$, and

$$
\begin{aligned}
& \varphi g_{2}=\left\langle\frac{t_{12}+t_{31} \bar{\varepsilon}}{t_{23}+1}\left(g_{2}+g_{3}\right)-g_{1}, \varepsilon g_{1}+g_{3}\right\rangle \frac{\varepsilon g_{1}+g_{3}}{t_{31}+1}-\frac{t_{12}+t_{31} \bar{\varepsilon}}{t_{23}+1}\left(g_{2}+g_{3}\right)+g_{1}= \\
= & \left(\frac{t_{12}+t_{31} \bar{\varepsilon}}{t_{23}+1}\left(t_{12} \bar{\varepsilon}+t_{31}+t_{23}+1\right)-\bar{\varepsilon}-t_{31} \bar{\varepsilon}\right) \frac{\varepsilon g_{1}+g_{3}}{t_{31}+1}-\frac{t_{12}+t_{31} \bar{\varepsilon}}{t_{23}+1}\left(g_{2}+g_{3}\right)+g_{1}= \\
= & \frac{\left(t_{12}+t_{31} \bar{\varepsilon}\right)\left(t_{12} \bar{\varepsilon}+t_{31}\right)+\left(t_{23}+1\right)\left(t_{12}-\bar{\varepsilon}\right)}{\left(t_{23}+1\right)\left(t_{31}+1\right)}\left(\varepsilon g_{1}+g_{3}\right)-\frac{t_{12}+t_{31} \bar{\varepsilon}}{t_{23}+1}\left(g_{2}+g_{3}\right)+g_{1} .
\end{aligned}
$$

Therefore, taking into account that $\left\langle g_{1}, g_{2}-t_{12} g_{1}\right\rangle=0$, we obtain

$$
\left(t_{23}+1\right)\left(t_{31}+1\right)\langle\varphi p, p\rangle=\left(t_{23}+1\right)\left(t_{31}+1\right)\left\langle\varphi g_{2}, g_{2}-t_{12} g_{1}\right\rangle=
$$




$$
\begin{gathered}
=\left(t_{23}+1\right)\left(t_{31}+1\right)\left\langle\frac{\left(t_{12}+t_{31} \bar{\varepsilon}\right)\left(t_{12} \bar{\varepsilon}+t_{31}\right)+\left(t_{23}+1\right)\left(t_{12}-\bar{\varepsilon}\right)}{\left(t_{23}+1\right)\left(t_{31}+1\right)} g_{3}-\frac{t_{12}+t_{31} \bar{\varepsilon}}{t_{23}+1}\left(g_{2}+g_{3}\right), g_{2}-t_{12} g_{1}\right\rangle= \\
=\left(t_{12}+t_{31} \bar{\varepsilon}\right)\left(t_{12} \bar{\varepsilon}+t_{31}\right)\left(t_{23}-t_{12} t_{31} \varepsilon\right)+\left(t_{23}+1\right)\left(t_{12}-\bar{\varepsilon}\right)\left(t_{23}-t_{12} t_{31} \varepsilon\right)- \\
\quad-\left(t_{12}+t_{31} \bar{\varepsilon}\right)\left(t_{31}+1\right)\left(1-t_{12}^{2}+t_{23}-t_{12} t_{31} \varepsilon\right)= \\
=t_{12} t_{23} t_{31} \bar{\varepsilon}^{2}+\left(t_{12}^{2} t_{23}+t_{12}^{2} t_{31}-t_{23}^{2}-t_{23} t_{31}-t_{31}^{2}-t_{23}-t_{31}\right) \bar{\varepsilon}+t_{12}^{3}+t_{12} t_{23}^{2}+t_{12} t_{23} t_{31}+t_{12} t_{31}^{2}-t_{12}-t_{12}^{2} t_{23} t_{31} \varepsilon .
\end{gathered}
$$

Hence,

$$
\begin{gathered}
\operatorname{Im}\left(-\left(t_{23}+1\right)\left(t_{31}+1\right)(1+\varepsilon)\langle\varphi p, p\rangle\right)=t_{12}^{2} t_{23} t_{31} \operatorname{Im} \varepsilon^{2}-t_{12} t_{23} t_{31} \operatorname{Im} \bar{\varepsilon}^{2}- \\
-\left(t_{12}^{2} t_{23}+t_{12}^{2} t_{31}+t_{12} t_{23} t_{31}-t_{23}^{2}-t_{23} t_{31}-t_{31}^{2}-t_{23}-t_{31}\right) \operatorname{Im} \bar{\varepsilon}- \\
-\left(t_{12}^{3}+t_{12} t_{23}^{2}+t_{12} t_{23} t_{31}+t_{12} t_{31}^{2}-t_{12}-t_{12}^{2} t_{23} t_{31}\right) \operatorname{Im} \varepsilon= \\
=\left(t_{12}+1\right) \varepsilon_{1}\left(1+2 t_{12} t_{23} t_{31} \varepsilon_{0}-t_{12}^{2}-t_{23}^{2}-t_{31}^{2}+\left(t_{12}-1\right)\left(t_{23}+1\right)\left(t_{31}+1\right)\right)= \\
=\left(t_{12}+1\right) \varepsilon_{1}\left(d+\left(t_{12}-1\right)\left(t_{23}+1\right)\left(t_{31}+1\right)\right) .
\end{gathered}
$$

By Lemma 4.4.11,

$$
\sqrt{2\left(1+\varepsilon_{0}\right)}\left(1-\frac{d}{\left(t_{12}+1\right)\left(t_{23}+1\right)\left(t_{31}+1\right)}\right)=2
$$

because the triangle is parabolic or trivial. So,

$$
d=\left(1-\frac{2}{\sqrt{2\left(1+\varepsilon_{0}\right)}}\right)\left(t_{12}+1\right)\left(t_{23}+1\right)\left(t_{31}+1\right)
$$

We see that the sign of $v$ is that of $\varepsilon_{1}\left(t_{12}-\frac{t_{12}+1}{\sqrt{2\left(1+\varepsilon_{0}\right)}}\right)$. By Criterion 4.4.2, $\varepsilon_{1}<0$ since the triangle is counterclockwise-oriented. It remains to prove that $0<t_{12}-\frac{t_{12}+1}{\sqrt{2\left(1+\varepsilon_{0}\right)}}$, i.e., that $2<\left(t_{12}-1\right)^{2}+2 t_{12}^{2} \varepsilon_{0}$. It suffices to show that $1<t_{12} \varepsilon_{0}$.

In order see that $1<t_{12} \varepsilon_{0}$, note that the inequality $1 \leq t_{23} t_{31}-\sqrt{\left(t_{23}^{2}-1\right)\left(t_{31}^{2}-1\right)}$ follows from $1<t_{23} t_{31}$. By Criterion 4.4.2, we have $t_{12}^{2} \varepsilon_{0}^{2}+t_{23}^{2}+t_{31}^{2}<1+2 t_{12} t_{23} t_{31} \varepsilon_{0}$ or, equivalently, $\left(t_{23} t_{31}-\right.$ $\left.t_{12} \varepsilon_{0}\right)^{2}<\left(t_{23}^{2}-1\right)\left(t_{31}^{2}-1\right)$. The inequality $t_{12} \varepsilon_{0} \leq t_{23} t_{31}$ implies $t_{23} t_{31}-\sqrt{\left(t_{21}^{2}-1\right)\left(t_{31}^{2}-1\right)}<t_{12} \varepsilon_{0}$.

4.4.17. $\mathbb{C}$-plane triangles. Assume that $d=0$. This means that the triangle of bisectors $\Delta\left(S_{1}, S_{2}, S_{3}\right)$ is built over a usual geodesic triangle $\Delta\left(p_{1}, p_{2}, p_{3}\right)$ situated in a complex geodesic $C$ of signature +- which is actually the common complex spine of the bisectors. In other words, $\mathrm{B}\left[S_{i}, S_{j}\right]=$ $\mathrm{B}\left[p_{i}, p_{j}\right]$, where $p_{i}$ is orthogonal to $g_{i}$ in $C$ (see Remark 4.1.10).

The triangle $\Delta\left(S_{1}, S_{2}, S_{3}\right)$ is always transversal. This follows from [Mos, p. 186, Lemma 2.3.4] (Mostow shows that the angle between cospinal bisectors is constant) or from Criterion 4.4.2: The inequality $t_{12}^{2} \varepsilon_{0}^{2}+t_{23}^{2}+t_{31}^{2}<1+2 t_{12} t_{23} t_{31} \varepsilon_{0}$ follows from $1+2 t_{12} t_{23} t_{31} \varepsilon_{0}=t_{12}^{2}+t_{23}^{2}+t_{31}^{2}$ and $\varepsilon_{0}^{2}<1$. (Note that $\varepsilon_{0}^{2}=1$ would imply that the points $g_{1}, g_{2}, g_{3}$ belong to the same geodesic by Lemma 4.1.13, implying that $p_{1}, p_{2}, p_{3}$ belong to the same geodesic by Remark 4.1.10.) Obviously, $\Delta\left(S_{1}, S_{2}, S_{3}\right)$ is counterclockwise-oriented exactly when $\Delta\left(p_{1}, p_{2}, p_{3}\right)$ is counterclockwise-oriented in $C$.

Lemma 4.4.18. Suppose that $\Delta\left(S_{1}, S_{2}, S_{3}\right)$ is counterclockwise-oriented and $\mathbb{C}$-plane. Then $\varphi$ (see 4.4.8 for the definition) restricted to $S_{1}$ is a rotation about $p_{1}$ by the angle -2 Area $\Delta\left(p_{1}, p_{2}, p_{3}\right)$.

Proof. Let $f \notin \overline{\mathbb{B}}$ denote the polar point to the complex geodesic $C$ containing $\Delta\left(p_{1}, p_{2}, p_{3}\right)$. Obviously, $\varphi f=-f$. By (4.4.10), $\varphi g_{1}=\varepsilon g_{1}$. Taking into account that the points $f, p_{1}, g_{1}$ form an orthogonal basis and that $\varphi \in \mathrm{SU}$, we obtain $\varphi p_{1}=-\bar{\varepsilon} p_{1}$. Assuming that $\langle f, f\rangle=1$ and $\left\langle p_{1}, p_{1}\right\rangle=-1$, 
we write every point in $S_{1} \cap \overline{\mathbb{B}}$ in the form $z f+p_{1},|z| \leq 1$. In terms of $z$, the isometry $\varphi$ acts on $S_{1} \cap \overline{\mathbb{B}}$ as the multiplication by $\varepsilon$. So, the restriction of $\varphi$ to $S_{1} \cap \overline{\mathbb{B}}$ is the rotation about $p_{1}$ by the angle $\arg \varepsilon \in[-\pi, \pi]$. It remains to show that 2 Area $\Delta\left(p_{1}, p_{2}, p_{3}\right)=-\arg \varepsilon$.

We apply to the representatives $\pi\left[g_{1}\right] g_{2}, \pi\left[g_{2}\right] g_{1}, \pi\left[g_{3}\right] g_{2}$ of $p_{1}, p_{2}, p_{3}$ the known formula

$$
\text { Area } \Delta\left(p_{1}, p_{2}, p_{3}\right)=\frac{1}{2} \arg \left(-\left\langle p_{1}, p_{2}\right\rangle\left\langle p_{2}, p_{3}\right\rangle\left\langle p_{3}, p_{1}\right\rangle\right)
$$

for the area of the oriented triangle $\Delta\left(p_{1}, p_{2}, p_{3}\right)$ (see [AGr1] or [Gol, p. 25]; by Corollary 4.1.18, the metric we use differs by the factor of 4 from the one in [Gol]) and obtain

$$
\begin{gathered}
2 \operatorname{Area} \Delta\left(p_{1}, p_{2}, p_{3}\right)=\arg \left(-\left\langle g_{2}-t_{12} g_{1}, g_{1}-t_{12} g_{2}\right\rangle\left\langle g_{1}-t_{12} g_{2}, g_{2}-t_{23} g_{3}\right\rangle\left\langle g_{2}-t_{23} g_{3}, g_{2}-t_{12} g_{1}\right\rangle\right)= \\
=\arg \left(-t_{12}\left(t_{12}^{2}-1\right) t_{23}\left(t_{12} t_{23}-t_{31} \bar{\varepsilon}\right)\left(1-t_{12}^{2}-t_{23}^{2}+t_{12} t_{23} t_{31} \varepsilon\right)\right)= \\
=\arg \left(\left(t_{12} t_{23}-t_{31} \bar{\varepsilon}\right)\left(t_{12}^{2}+t_{23}^{2}-t_{12} t_{23} t_{31} \varepsilon-1\right)\right) .
\end{gathered}
$$

It follows from $1+2 t_{12} t_{23} t_{31} \varepsilon_{0}=t_{12}^{2}+t_{23}^{2}+t_{31}^{2}$ that

$$
t_{12}^{2}+t_{23}^{2}-t_{12} t_{23} t_{31} \varepsilon-1=2 t_{12} t_{23} t_{31} \varepsilon_{0}-t_{12} t_{23} t_{31} \varepsilon-t_{31}^{2}=t_{31}\left(t_{12} t_{23} \bar{\varepsilon}-t_{31}\right)=t_{31}\left(t_{12} t_{23}-t_{31} \varepsilon\right) \bar{\varepsilon},
$$

which implies the result.

\subsection{Kähler primitive}

The hermitian form (4.1.2) defines a Kähler form $\omega$ on $\mathbb{P} \backslash \partial_{\infty} \mathbb{B}$ by the rule $\omega\left(v_{p}, w_{p}\right):=\operatorname{Im}\left\langle v_{p}, w_{p}\right\rangle$, where $v_{p}, w_{p} \in \mathrm{T}_{p} \mathbb{P}$ (see (4.1.3) for the definition of $v_{p}$ ). In this subsection, we obtain a primitive $P_{c}$ for $\omega$ that depends on the choice of a base point $c$ and study how this primitive changes when we alter the base point.

4.5.1. Let $c \in \mathbb{P}$, let $p \in \mathbb{P} \backslash\left(\mathbb{P} c^{\perp} \cup \partial_{\infty} \mathbb{B}\right)$, and let $v_{p} \in \mathrm{T}_{p} \mathbb{P}$. Define

$$
P_{c}\left(v_{p}\right):=-\operatorname{Im}\left(\frac{\langle p, p\rangle\langle v, c\rangle}{2\langle p, c\rangle}\right) .
$$

Note that $P_{c}\left(v_{p}\right)$ does not depend on the choice of representatives $c, p, v \in V$ that give the same $v_{p}$. Obviously, $P_{c}\left(v_{p}\right)$ depends smoothly on $v_{p} \in \mathrm{T}_{p} \mathbb{P}$, i.e., it defines a 1-form on $\mathbb{P} \backslash\left(\mathbb{P} c^{\perp} \cup \partial_{\infty} \mathbb{B}\right)$.

Let $c_{1}, c_{2} \in \mathbb{P}$ be nonorthogonal. Define the multi-valued function

$$
f_{c_{1}, c_{2}}(p):=\frac{1}{2} \operatorname{Arg}\left(\frac{\left\langle c_{1}, p\right\rangle\left\langle p, c_{2}\right\rangle}{\left\langle c_{1}, c_{2}\right\rangle}\right)
$$

where $p \in \mathbb{P} \backslash\left(\mathbb{P} c_{1}^{\perp} \cup \mathbb{P} c_{2}^{\perp}\right)$. Note that $f_{c_{1}, c_{2}}(p)$ does not depend on the choice of representatives $c_{1}, c_{2}, p \in V$.

Proposition 4.5.2. For nonorthogonal $c_{1}, c_{2} \in \mathbb{P}$, we have $P_{c_{1}}-P_{c_{2}}=d f_{c_{1}, c_{2}}$ on $\mathbb{P} \backslash\left(\partial_{\infty} \mathbb{B} \cup \mathbb{P} c_{1}^{\perp} \cup \mathbb{P} c_{2}^{\perp}\right)$.

Proof. Choose representatives $c_{1}, c_{2} \in V$ such that $\left\langle c_{1}, c_{2}\right\rangle \in \mathbb{R}$. Take $p \in \mathbb{P} \backslash\left(\partial_{\infty} \mathbb{B} \cup \mathbb{P} c_{1}^{\perp} \cup \mathbb{P} c_{2}^{\perp}\right)$ and $v_{p} \in \mathrm{T}_{p} \mathbb{P}$. Then

$$
\begin{gathered}
d f_{c_{1}, c_{2}}\left(v_{p}\right)=\left.\frac{d}{d \varepsilon}\right|_{\varepsilon=0} f_{c_{1}, c_{2}}(p+\varepsilon\langle p, p\rangle v)=\left.\frac{1}{2} \frac{d}{d \varepsilon}\right|_{\varepsilon=0} \operatorname{Arg}\left(\left\langle c_{1}, p+\varepsilon\langle p, p\rangle v\right\rangle\left\langle p+\varepsilon\langle p, p\rangle v, c_{2}\right\rangle\right)= \\
\quad=\left.\frac{1}{2} \operatorname{Im} \frac{d}{d \varepsilon}\right|_{\varepsilon=0}\left(\ln \left\langle c_{1}, p+\varepsilon\langle p, p\rangle v\right\rangle+\ln \left\langle p+\varepsilon\langle p, p\rangle v, c_{2}\right\rangle\right)=
\end{gathered}
$$




$$
=\frac{1}{2} \operatorname{Im}\left(\frac{\langle p, p\rangle\left\langle c_{1}, v\right\rangle}{\left\langle c_{1}, p\right\rangle}+\frac{\langle p, p\rangle\left\langle v, c_{2}\right\rangle}{\left\langle p, c_{2}\right\rangle}\right)=\left(P_{c_{1}}-P_{c_{2}}\right)\left(v_{p}\right) .
$$

4.5.3. In order to show that $P_{c}$ is a primitive for the form $\omega$, we introduce an auxiliary vector field. Let $p \in \mathbb{P} \backslash \partial_{\infty} \mathbb{B}$ and let $v_{p} \in \mathrm{T}_{p} \mathbb{P}$. The smooth vector field

$$
\operatorname{Tn}\left(v_{p}\right)(x):=\langle-, x\rangle \frac{\langle p, p\rangle \pi[x] v}{\langle p, x\rangle}
$$

is defined on $\mathbb{P} \backslash\left(\mathbb{P} p^{\perp} \cup \partial_{\infty} \mathbb{B}\right)$. This field extends the vector $v_{p}$, i.e., $\operatorname{Tn}\left(v_{p}\right)(p)=v_{p}$. Note that $\operatorname{Tn}\left(v_{p}\right)(x)$ does not depend on the choice of representatives $p, v, x \in V$ that give the same $v_{p}$.

Lemma 4.5.4. Let $p \in \mathbb{P} \backslash \partial_{\infty} \mathbb{B}$ and let $v_{p}, w_{p} \in \mathrm{T}_{p} \mathbb{P}$. Then $\left[\operatorname{Tn}\left(v_{p}\right), \operatorname{Tn}\left(w_{p}\right)\right](p)=0$.

Proof. Denote by $\hat{f}$ the lift to $V$ of a $C^{2}$-function $f$ defined in a neighbourhood of $p$. So, $\hat{f}(u x)=\hat{f}(x)$ for $0 \neq u \in \mathbb{C}$. By definition,

$$
\operatorname{Tn}\left(w_{p}\right) f(x)=\left.\frac{d}{d \varepsilon}\right|_{\varepsilon=0} \hat{f}\left(x+\varepsilon\langle x, x\rangle \frac{\langle p, p\rangle \pi[x] w}{\langle p, x\rangle}\right) .
$$

Therefore,

$$
\begin{gathered}
\operatorname{Tn}\left(v_{p}\right)\left(\operatorname{Tn}\left(w_{p}\right) f\right)(p)= \\
=\left.\frac{d}{d \delta}\right|_{\delta=0}\left(\left.\frac{d}{d \varepsilon}\right|_{\varepsilon=0} \hat{f}\left(p+\delta\langle p, p\rangle v+\varepsilon\langle p+\delta\langle p, p\rangle v, p+\delta\langle p, p\rangle v\rangle \frac{\langle p, p\rangle \pi[p+\delta\langle p, p\rangle v] w}{\langle p, p+\delta\langle p, p\rangle v\rangle}\right)\right)= \\
=\left.\frac{d}{d \delta}\right|_{\delta=0}\left(\left.\frac{d}{d \varepsilon}\right|_{\varepsilon=0} \hat{f}\left(p+\delta\langle p, p\rangle v+\varepsilon\left(\langle p, p\rangle+\delta^{2}\langle p, p\rangle^{2}\langle v, v\rangle\right) w-\varepsilon \delta\langle p, p\rangle\langle w, v\rangle(p+\delta\langle p, p\rangle v)\right)\right)= \\
=\left.\frac{d}{d \delta}\right|_{\delta=0}\left(\left.\frac{d}{d \varepsilon}\right|_{\varepsilon=0} \hat{f}\left((1-\varepsilon \delta\langle p, p\rangle\langle w, v\rangle)(p+\delta\langle p, p\rangle v)+\varepsilon\left(\langle p, p\rangle+\delta^{2}\langle p, p\rangle^{2}\langle v, v\rangle\right) w\right)\right)= \\
\left.\frac{d}{d \delta}\right|_{\delta=0}\left(\left.\frac{d}{d \varepsilon}\right|_{\varepsilon=0} \hat{f}\left(p+\delta\langle p, p\rangle v+\varepsilon\langle p, p\rangle \frac{1+\delta^{2}\langle p, p\rangle\langle v, v\rangle}{1-\varepsilon \delta\langle p, p\rangle\langle w, v\rangle} w\right)\right)=\left.\frac{d}{d \delta}\right|_{\delta=0}\left(\left.\frac{d}{d \varepsilon}\right|_{\varepsilon=0} \hat{f}(p+\delta\langle p, p\rangle v+\varepsilon\langle p, p\rangle w)\right) .
\end{gathered}
$$

By symmetry,

$$
\operatorname{Tn}\left(w_{p}\right)\left(\operatorname{Tn}\left(v_{p}\right) f\right)(p)=\left.\frac{d}{d \delta}\right|_{\delta=0}\left(\left.\frac{d}{d \varepsilon}\right|_{\varepsilon=0} \hat{f}(p+\delta\langle p, p\rangle w+\varepsilon\langle p, p\rangle v)\right) .
$$

Lemma 4.5.5. Let $c \in \mathbb{P}$, let $p \in \mathbb{P} \backslash\left(\mathbb{P} c^{\perp} \cup \partial_{\infty} \mathbb{B}\right)$, and let $v_{p}, w_{p} \in \mathrm{T}_{p} \mathbb{P}$. Then

$$
v_{p} P_{c}\left(\operatorname{Tn}\left(w_{p}\right)\right)=\frac{\langle p, p\rangle}{2} \operatorname{Im}\left(\langle w, v\rangle+\frac{\langle p, p\rangle\langle v, c\rangle\langle w, c\rangle}{\langle p, c\rangle^{2}}\right) .
$$

Proof. By definition,

$$
\begin{gathered}
P_{c}\left(\operatorname{Tn}\left(w_{p}\right)\right)(x)=-\operatorname{Im} \frac{\langle x, x\rangle\langle p, p\rangle\langle\pi[x] w, c\rangle}{2\langle x, c\rangle\langle p, x\rangle}= \\
=-\operatorname{Im} \frac{\langle x, x\rangle\langle p, p\rangle\langle w, c\rangle-\langle p, p\rangle\langle w, x\rangle\langle x, c\rangle}{2\langle x, c\rangle\langle p, x\rangle}=\frac{\langle p, p\rangle}{2} \operatorname{Im}\left(\frac{\langle w, x\rangle}{\langle p, x\rangle}-\frac{\langle x, x\rangle\langle w, c\rangle}{\langle p, x\rangle\langle x, c\rangle}\right) .
\end{gathered}
$$


Therefore,

$$
\begin{gathered}
v_{p} P_{c}\left(\operatorname{Tn}\left(w_{p}\right)\right)=\left.\frac{\langle p, p\rangle}{2} \frac{d}{d \varepsilon}\right|_{\varepsilon=0} \operatorname{Im}\left(\frac{\langle w, p+\varepsilon\langle p, p\rangle v\rangle}{\langle p, p+\varepsilon\langle p, p\rangle v\rangle}-\frac{\langle p+\varepsilon\langle p, p\rangle v, p+\varepsilon\langle p, p\rangle v\rangle\langle w, c\rangle}{\langle p, p+\varepsilon\langle p, p\rangle v\rangle\langle p+\varepsilon\langle p, p\rangle v, c\rangle}\right)= \\
=\left.\frac{\langle p, p\rangle}{2} \frac{d}{d \varepsilon}\right|_{\varepsilon=0} \operatorname{Im}\left(\varepsilon\langle w, v\rangle-\frac{1+\varepsilon^{2}\langle p, p\rangle\langle v, v\rangle}{\langle p, c\rangle+\varepsilon\langle p, p\rangle\langle v, c\rangle}\langle w, c\rangle\right)=\frac{\langle p, p\rangle}{2} \operatorname{Im}\left(\langle w, v\rangle+\frac{\langle p, p\rangle\langle v, c\rangle\langle w, c\rangle}{\langle p, c\rangle^{2}}\right) .
\end{gathered}
$$

Proposition 4.5.6. For every $c \in \mathbb{P}$, we have $d P_{c}=\omega$ on $\mathbb{P} \backslash\left(\partial_{\infty} \mathbb{B} \cup \mathbb{P} c^{\perp}\right)$.

Proof. Let $p \in \mathbb{P} \backslash\left(\partial_{\infty} \mathbb{B} \cup \mathbb{P}^{\perp}\right)$ and let $v_{p}, w_{p} \in \mathrm{T}_{p} \mathbb{P}$. Take $X:=\operatorname{Tn}\left(v_{p}\right)$ and $Y:=\operatorname{Tn}\left(w_{p}\right)$. By Lemma 4.5.4, $[X, Y](p)=0$. Hence, $P_{c}([X, Y])(p)=0$. By the Maurer-Cartan identity,

$$
d P_{c}(X, Y)(p)=X\left(P_{c}(Y)\right)(p)-Y\left(P_{c}(X)\right)(p)-P_{c}([X, Y])(p) .
$$

It follows from Lemma 4.5.5 that

$$
\begin{gathered}
d P_{c}\left(v_{p}, w_{p}\right)=\frac{\langle p, p\rangle}{2} \operatorname{Im}\left(\langle w, v\rangle+\frac{\langle p, p\rangle\langle v, c\rangle\langle w, c\rangle}{\langle p, c\rangle^{2}}\right)-\frac{\langle p, p\rangle}{2} \operatorname{Im}\left(\langle v, w\rangle+\frac{\langle p, p\rangle\langle w, c\rangle\langle v, c\rangle}{\langle p, c\rangle^{2}}\right)= \\
=\frac{1}{2} \operatorname{Im}(\langle p, p\rangle\langle w, v\rangle-\langle p, p\rangle\langle v, w\rangle)=\operatorname{Im}\left\langle v_{p}, w_{p}\right\rangle .
\end{gathered}
$$

\section{References}

[AGr1] S. Anan'in, C. H. Grossi, Coordinate-free classic geometries, Mosc. Math. J., to appear, see also http://arxiv.org/abs/math/0702714.

[AGr2] S. Anan'in, C. H. Grossi, Invariants of finite configurations in $\mathbb{H}_{\mathbb{C}}^{2}$ and their geometric interpretation, in preparation.

[AGr3] S. Anan'in, C. H. Grossi, Yet another Poincaré polyhedron theorem, Proc. Edinburgh Math. Soc. 54 (2011), 297-308, see also http://arxiv.org/abs/0812.4161.

[BSi] F. Bonahon, L. Siebenmann, The classification of Seifert fibered 3-orbifolds, in Low Dimensional Topology, edited by R. Fenn, London Mathematical Society LNS 95, Cambridge University Press, New York, 1985, 258 pp.

[GKL] W. M. Goldman, M. Kapovich, B. Leeb, Complex hyperbolic manifolds homotopy equivalent to a Riemann surface, Comm. Anal. Geom. 9, no. 1 (2001), 61-95.

[GLT] M. Gromov, H. B. Lawson Jr., W. Thurston, Hyperbolic 4-manifolds and conformally flat 3-manifolds, Inst. Hautes Études Sci. Publ. Math. 68 (1988), 27-45.

[Gol] W. M. Goldman, Complex hyperbolic geometry, Oxford Mathematical Monographs. Oxford Science Publications. The Clarendon Press, Oxford University Press, New York, 1999, xx+316 pp.

[HSa] J. Hakim, H. Sandler, Application of Bruhat decomposition to complex hyperbolic geometry, J. Geom. Anal. 10 (2000), 435-453.

[Hsi] Hsieh, Po-Hsun, Cotranchal bisectors in complex hyperbolic space, Geometriae Dedicata 97 (2003), 93-98.

[Kap1] M. Kapovich, Flat conformal structures on 3-manifolds. I. Uniformization of closed Seifert manifolds, J. Differential Geom. 38, no. 1 (1993), 191-215.

[Kap2] M. Kapovich, On hyperbolic 4-manifolds fibered over surfaces, preprint (1993). Available at http://www.math.ucdavis.edu/ kapovich/eprints.html.

[KoM] A. I. Kostrikin, Y. I. Manin, Linear algebra and geometry, Algebra, Logic and Applications Series. Gordon and Breach Science Publishers, London, 1989, xvi+309 pp. 
[Kre] M. Krebs, Toledo invariants of 2-orbifolds and Higgs bundles on elliptic surfaces, Michigan Math. J. 56, no. 1 (2008), 3-27.

[Kui] N. H. Kuiper, Hyperbolic 4-manifolds and tessellations, Inst. Hautes Études Sci. Publ. Math. 68 (1988), 47-76.

[Luo] F. Luo, Constructing conformally flat structures on some Seifert fibred 3-manifolds, Math. Ann. 294, no. 3 (1992), 449-458.

[Pra] A. Pratoussevitch, Traces in complex hyperbolic triangle groups, Geometriae Dedicata 111 (2005), 159-185.

[San] H. Sandler, Distance formulas in complex hyperbolic space, Forum Math. 8, no. 1 (1996), 93-106.

[Sch] S. Schleimer, Waldhausen's theorem, Geom. Topol. Monographs 12 (2007), 299-317.

[Tol] D. Toledo, Representations of surface groups in complex hyperbolic space, J. Differential Geom. 29, no. 1 (1989), 125-133.

[Xia] E. Z. Xia, The moduli of flat $\mathrm{PU}(2,1)$ structures on Riemann surfaces, Pacific J. Math. 195, no. 1 (2000), 231-256.

Departamento de Matemática, imecC, Universidade Estadual de Campinas, 13083-970-CAMPINAS-SP, BRASIL

Max-Planck-Institut für Mathematik, Vivatsgasse 7, 53111 Bonn, Germany

E-mail address: grossi_ferreira@yahoo.com

Departamento de Matemática, ICEX, Universidade Federal de Minas Gerais, 31161-970-Belo Horizonte-MG, Brasil 